\title{
Simulación Dinámica y Control de Reactores Tubulares mediante
}

Formulaciones de Ecuaciones Integrales

Tesis que presenta

I. Q. Eliseo Hernández Martínez

Para obtener el grado de

Maestro en Ciencias (Ingeniería Química)

Asesores:

Dr. José de Jesus Álvarez Ramírez

Dr. Jesús Álvarez Calderón

Noviembre, 2008 


\section{Dedicado}

a mi querida esposa

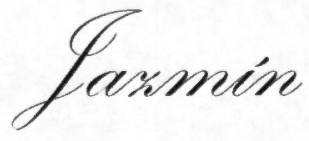

y a mis dos pequeños Stepany e. Paxc 


\section{Agradecimientos:}

Primero que nada quiero agradecer a Dios par haberme brindado la capacidad para realizar este proyecto de investigación.

Al Consejo Nacional de Ciencia y Tecnología por haberme brindado apoyo económico durante estos dos años, con registro 205866/205252.

A mis asesores de tesis Dr. José Álvarez Ramírez y Dr. Jesús Álvarez Calderón por sus valiosos consejos y sus tan acertadas criticas que fortalecieron este trabajo. Al mismo tiempo quiero agradecer por su valioso tiempo y apoyo al Dr. Héctor Puebla de UAM-A ya que gran parte de mi aprendizaje en este nivel se lo debo a el.

Quiero hacer un reconocimiento a mis revisores de tesis Dr. Ramiro Rico Martínez del Tecnológico de Celaya, a los Drs. José Álvarez Ramírez y el Dr. Jesús A. Ochoa de la UAMIztapalapa por su tiempo y comentarios que fortalecieron el contenido de este trabajo,

Este trabajo no solo es mío si no también es de las personas que estado conmigo en cada una de la etapas de su desarrollo, me refiero a mi querida esposa Jazmín que me ha sabido comprender y ha respetado todas las decisiones que he tomado a lo largo de los 9 años de matrimonio que llevamos, ;Gracias por todo, mi amor! A mi hija Stefany que ha pesar de ser tan pequeña entiende el camino que he tomado y me apoya con su cariño y a mi pequeño bebe Isaac que es el motor de mis acciones ¡Gracias mis pequeños!

Una parte importante para la culminación de este trabajo es gracias a mi querida familia que siempre ha estado conmigo que me ha provisto de aliento y apoyo en los momentos mas difíciles, a mi Padre Pedro Hernández por su ejemplo de dedicación, a mi Madre Angélica Martínez por su cariño y a mis hermanos Ricardo y Gabriel que han sido una gran fuente de inspiración.

Quiero agradecer a mis compañeros de generación, que más que mis compañeros son mis amigos, a Luis (wipi), Agustín, Matías, Dafnis, Rocha, Alex, Gustavo, Páramo y en especial a Vicky que gracias a sus consejos he podido culminar esta etapa de mi preparación académica. Finalmente quiero agradecer a mis profesores (Dr. Lobo, Dr. Revah, Dr. Zurita, Dr. Viveros, Dr. Soria, por mencionar algunos) por haber compartido un poco de su experiencia conmigo y aunque en ocasiones creía que eran muy duros ahora comprendo que tenia que ser así. 


\section{Resumen General}

En este trabajo se explora el uso de formulaciones de ecuaciones integrales (FEIs) para la solución numérica de modelos que describen el comportamiento de reactores tubulares, con el objeto de implementación de algoritmos de control. Las formulaciones de ecuaciones integrales son básicamente una transformación de ecuaciones diferenciales a ecuaciones integrales. Las FEIs tiene ventajas numéricas frente a esquemas de discretización clásicos (diferencias finitas y colocación ortogonal) tales como: $i$ ) reducen el mallado y con ello reduce el tiempo de cómputo, ii) son estables en todo el rango de operación e iii) incorpora las condiciones de frontera de manera exacta en las ecuaciones integrales resultantes.

El objetivo primordial del control en reactores tubulares es la regulación de la concentración a la salida del reactor manteniendo la temperatura debajo de los valores máximos especificados. Dado que la medición de la composición tiene generalmente retardos, debido a las restricciones en la respuestas de los dispositivos de medición y al transporte interno, un lazo retroalimentado, basado únicamente en la medición de la composición puede sufrir un pobre desempeño y hasta inestabilidades. Para aliviar esta situación se han empleado esquemas de control en cascada composición-temperatura. Se han reportado en la literatura sofisticados esquemas de control, pero debido a su complejidad y a la gran dependencia del modelo matemático están limitados para su aplicación industrial. Por otro lado los compensadores PI/PID son bien aceptados en la industria debido a su facilidad de diseño e implementación. En este trabajo extendemos el uso del controlador en cascada basado en compensadores PI/PID, empleando la medición de tres sensores de temperatura distribuidos simétricamente a lo largo de la posición axial del reactor tubular. La configuración del controlador explota la información provista por dos sensores de temperatura adicionales localizados cerca de la entrada y salida del reactor lo que permite al reactor una respuesta mas rápida a lazo cerrado. El desempeño del controlador se muestra mediante simulaciones numéricas en un reactor tubular con dos dinámicas diferentes: con y sin hot spot. 


\section{Índice General}

\section{Capítulo I. Introducción}

1.1. Motivación

1.2. PDE y Métodos Numéricos

1.3. Estado de Arte 10

1.3.1. Uso de Funciones de Green en procesos Quimicos 11

$\begin{array}{ll}\text { 1.3.2. Control de Reactores Tubulares } & 13\end{array}$

1.4. Definición del Problema $\quad \ldots \quad 14$

$\begin{array}{ll}\text { 1:5. Objetivos } & 14\end{array}$

$\begin{array}{ll}\text { 1.5.1. Objetivo General } & 14\end{array}$

1.5.2. Objetivos Particulares

\section{Capítulo II. Formulación de Ecuaciones Integrales}

2.1 Introducción 15

$\begin{array}{ll}2.2 \text { Operadores diferenciales lineales } & 16 \\ & 17\end{array}$

$\begin{array}{ll}\text { 2.2.1 Operador Sturm-Liouville } & 19\end{array}$

$\begin{array}{ll}2.3 \mathrm{La} \text { función de Green } & 20\end{array}$

$\begin{array}{ll}\text { 2.3.1 Construcción de la función de Green } & 21\end{array}$

2.3.2 Método Alternativo para el cálculo de la función de Green

\section{Capitulo III Estrategia de Control}

3.1 Control de procesos

3.2 Control en cascada

3.2.1 Antecedentes 25

3.2.2 Cuando usar control en cascada

$\begin{array}{ll}3.3 \text { Sintonizado del controlador } & 27\end{array}$

3.4 Diseño del controlador en cascada para reactores tubulares

3.4.1 Diseño del controlador 28

3.4.2 Control en cascada convencional

3.4.2.1 Lazo de composición 29

3.4.2.2 Lazo de temperatura 30

3.4.3 Diseño de control en cascada con temperatura promedio

\section{Capítulo IV. Simulación a lazo abierto}

4.1 Reactor tubular con dispersión axial

4.2 Modelo del reactor tubular 33

4.3 Formulación del Reactor Tubular en Ecuaciones Integrales 35

4.3 Formulación del Reactor Tubular en Ecuaciones Integrales 38 
4.5 Dinámica a lazo abierto $\quad 39$

$\begin{array}{ll}\text { 4.5.1 Perfil estable y cuasi-lineal } & 40\end{array}$

4.5.2 Perfil estable con hot spot $\quad 41$

4.6 Análisis numérico 43

4.6.1 Análisis en estado estacionario 43

4.6.1.1 Perfil de temperatura cuasi-lineal $\quad 44$

4.6.1.2 Perfil de temperatura con hot spot 46

4.6.2 Análisis dinámico $\quad 49$

4.6.2.1 Perfil de temperatura cuasi-lineal $\quad 49$

4.6.2.1 Perfil de temperatura con hot spot $\quad 53$

Capitulo V. Simulación a lazo cerrado

5.1 Introducción 56

5.2 Análisis Dinámico $\quad 57$

5.3 Dinámica a lazo cerrado $\quad \ldots \quad 60$

$\begin{array}{ll}\text { 5.3.1 Control en cascada convencional } & 60\end{array}$

$\begin{array}{ll}\text { 5.3.1.1 Perfil de temperatura cuasi-lineal } & 60\end{array}$

5.3.1.2 Perfil temperatura con hot spot 63

5.3.2 Control en cascada con temperatura promedio ponderada 63

$\begin{array}{ll}\text { 5.3.2.1 Perfil estable y cuasi-lineal } & 63\end{array}$

5.3.3.2 Perfil estable con hot spot 64

5.3.3 Comparación de los esquemas de control 65

5.4 Pruebas de Robustez $\quad 69$

Conclusiones $\quad 71$

$\begin{array}{ll}\text { Bibliografía } & 73\end{array}$

Apéndice

$\begin{array}{ll}\text { A.1 Propiedades de la función Impulso } & 78\end{array}$

$\begin{array}{lr}\text { A.2 Diferencias Finitas } & 80\end{array}$

A.3 Colocación Ortogonal $\quad 81$ 


\section{Índice de figuras}

Figura 3-1 Diagrama de bloques de control en cascada 24

Figura 3-2 Representación del control en cascada del reactor tubular 28

Figura 4-1 Perfiles de concentración y temperatura adimensional (perfil cuasi-lineal) 41

Figura 4-2 Perfiles de concentración y temperatura adimensional (perfil con hot spot) $\quad 42$

Figura 4-3 Perfil de temperatura cuasi lineal a diferentes números de Peclet en estado $\quad 44$

estacionario

Figura 4-4 Aproximación numérica, caso: perfil cuasi lineal, error relativo vs. 45

numero de nodos

Figura 4-5 Perfil de temperatura con hot spot a diferentes números de Peclet en

estado estacionario

Figura 4-6 Aproximación numérica, caso: perfil con hot spot, error relativo vs. numero de nodos

Figura 4-7 Perfil de temperatura cuasi lineal $(\xi=1)$ a diferentes números de Peclet en estado dinámico

Figura 4-8 Solución dinámica del reactor tubular en 3 dimensiones a diferentes valores de Peclet, perfil cuasi lineal

Figura 4-9 Aproximación numérica dinámica, caso: perfil cuasi lineal, error relativo vs. numero de nodos

Figura 4-10 Perfil de temperatura cuasi lineal $(\xi=1)$ a diferentes números de Peclet en estado dinámico

Figura 4-11 Solución dinámica del reactor tubular en 3 dimensiones a diferentes valores de Peclet, perfil con hot spot

Figura 4-12 Aproximación numérica dinámica, caso: perfil con hot spot, error relativo vs. numero de nodos,

Figura 5-1 Respuestas a perturbación en +10 en $\varpi$

Figura 5-2 Respuestas a perturbación en +10 en $\varpi$ a $t=10$, dinámica sin hot spot

Figura 5-3 Respuestas a perturbación en $+10 \%$ en ш a t=10, dinámica con hot spot

Figura 5-4 Control en cascada convencional con sensor de temperatura en posición adimensional 0.20 .5 y 0.8 : perfil cuasi-lineal

Figura 5-5 Control en cascada convencional con sensor de temperatura en posición adimensional 0.20 .5 y 0.8 : perfil con hot spot

Figura 5-6 Integral del error al cuadrado de la respuesta a perturbaciones del controlador en cascada con $w_{a v}$

Figura 5-7 Integral del error al cuadrado de la respuesta a perturbaciones del controlador en cascada con $w_{a v}$

Figura 5-8 Desempeño de esquemas de control, un solo lazo de retroalimentación PI, control en cascada clásico y control en cascada propuesto: perfil cuasi-lineal

Figura 5-9 Desempeño de esquemas de control, un solo lazo de retroalimentación PI, control en cascada clásico y control en cascada propuesto

Figura 5-10 Pruebas de robustez al controlador propuesto, variación el los parámetros del modelo, perfil cuasi-lineal

Figura 5-11 Pruebas de robustez al controlador propuesto, variación el los parámetros del modelo. Perfil con hot spot

Figura A-1 Representación constante a trozos de una función 


\section{Índice de Tablas}

Pag.

Tabla 4-1 Parámetros para simulación del reactor, perfil de temperatura cuasilineal

Tabla 4-2 Parámetros para simulación del reactor, perfil de temperatura con hot spot

Tabla 4-3 Tiempos de cómputo, perfil de temperatura cuasi-lineal

Tabla 4-4 Tiempos de cómputo, perfil de temperatura con hot spot

Tabla 4-5 Tiempos de cómputo, perfil dinámico de temperatura cuasi-lineal

Tabla 4-6 Tiempos de cómputo, perfil dinámico de temperatura con hot spot 


\section{Capítulo 1}

\section{Introducción}

En este capitulo se presenta la motivación para la elaboración de esta tesis, el estado del arte de los métodos de solución numérica y control de los reactores tubulares planteando asi la definición del problema a abordar y finalmente se definen los objetivos generales y particulares de este trabajo.

\subsection{Motivación}

Los principales reactores en la industria química son el reactor tanque agitado y el reactor tubular. Para algunas reacciones químicas los reactores tubulares son más eficientes que los reactores tanque agitado, especialmente cuando los volúmenes son iguales. Además el reactor tubular puede operarse a largos tiempo sin necesidad de mantenimiento y usualmente produce mejores conversiones por unidad de volumen que cualquier reactor de flujo continuo. El reactor químico tubular físicamente tiene forma de un recipiente cilíndrico por el que los reactantes fluyen, no existe mezclado inducido en ningún punto de la dirección espacial del reactor. Los reactantes entran de manera continua en un extremo del reactor, la reacción se llevan acabo a medida que estos se desplazan en la longitud axial del reactor saliendo así reactantes y productos al final del reactor (Froment \& Bischoff, 1990). A nivel industrial los reactores tubulares se utilizan en procesos como la producción de polietileno de baja densidad, benceno, tolueno, crackeo de hidrocarburos, etanol, acido acético, etileno, nylon-6, polipropileno, etc. (Smith, 1986)

El modelo matemático que describe el comportamiento dinámico de los reactores tubulares es descrito por ecuaciones diferenciales parciales (EDP) no lineales (sistemas de parámetros distribuidos (SPD)). De acuerdo al tipo de problema que se analice las (EDP) pueden clasificarse como parabólicas o hiperbólicas. En problemas donde el transporte por difusión tiene gran impacto (reacción-difusión-convección o reacción-difusión) tenemos un sistema en (EDP) parabólicas y cuando solo es considerado el transporte por convección (reacciónconvección) tenemos (EDP) hiperbólicas (Christofides, 2001). Los sistemas en el cual la reacción química es acoplada con fenómenos de transporte difusión-convección surgen en una 
gran gama de aplicaciones en ingeniería química y requieren de sofisticadas herramientas matemáticas para su estudio riguroso (Khalid et al., 2003).

La aproximación de un conjunto de (EDPs) a un conjunto de ecuaciones diferenciales ordinarias (EDOs) es necesaria para reducir una excesiva demanda de tiempo de cómputo que se requiere para la solución de modelos con alta dimensionalidad. El sistema reducido (discreto) obtenido puede ser una buena aproximación al (SPD) original (continuo) solo si los parámetros, como el intervalo de tiempo, el mallado espacial, la distribución de nodos, etc. de las técnicas de reducción se seleccionan adecuadamente (Lefevre et al., 2000).

Los métodos de discretización clásicos empleados en problemas de ingeniería química son diferencias finitas y colocación ortogonal (Rice \& Do, 1995). Sin embargo se ha demostrado que el uso de estas técnicas de discretización pueden no ser estables para un rango de valores como se requieren, tal es el caso en modelos de estimación de parámetros de acuerdo a Agrawal et al. (2006).

Dada la importancia del uso de los reactores tubulares en la industria química y debido a la existencia de sensibilidad paramétrica, y comportamientos altamente no lineales, que son producto del acoplamiento intrínsico de los fenómenos de transporte y la reacción química, puede conducir a condiciones de operación no adecuadas, incluso a la destrucción misma del reactor o de la planta, por lo que es necesario el desarrollo de esquemas de control para este tipo de reactores que permitan una operación eficiente y segura.

En años recientes, la demanda de la calidad de los productos industriales ha ido en aumento, por lo que cada vez se requieren sistemas de optimización y control más robustos que cumplan con las demandas establecidas. La simulación dinámica para el diseño de algoritmos de control y optimización implica una gran reducción de costos en la producción a nivel industrial (Li y Christofides, 2007), por lo que es imprescindible contar con modelos matemáticos confiables que describan de manera mas precisa el comportamiento físico de los sistemas y al mismo tiempo contar con metodologías de solución numéricas estables que aminoren las incertidumbre de los modelos.

\subsection{Ecuaciones diferenciales parciales y métodos numéricos}

Debido a su aplicación extendida, las soluciones de las EPDs han sido un tema de estudio en el campo de la ingeniería. Los métodos de solución existentes se pueden dividir en métodos analíticos y métodos numéricos. Los métodos analíticos están restringidos a la solución de problemas lineales, sin embargo, debido a que la gran mayoría de los problemas que se abordan en ingeniería son modelos con ecuaciones no lineales, el uso de los métodos analíticos esta limitado. Con el acelerado desarrollo computacional de los últimos años no es raro que el uso de los métodos numéricos en las matemáticas aplicadas y la ingeniería, haya cobrado mayor relevancia para resolver problemas complejos para los cuales no se dispone, hasta el momento de soluciones analíticas.

Entre los métodos numéricos más importantes para resolver ecuaciones diferenciales se tienen el método de colocación ortogonal (CO) (Villadsen \& Stewart, 1967; Rice \& Do, 1995; Finlayson, 1980) y el método de diferencias finitas (DF) (Smith, 1978; Rice \& Do, 1995), entre otros. El método de diferencias finitas consiste en una aproximación al modelo diferencial, que se logra sustituyendo los diferentes operadores diferenciales (de primer orden, 
segundo orden o mayor) por operadores en diferencias (empleando series de Taylor), transformando de esta manera la ecuación diferencial en una ecuación algebraica. Para obtener una aproximación adecuada, las diferencias finitas deben ser lo suficientemente pequeñas (conforme a la teoría de límites), razón por la cual el dominio de la ecuación diferencial debe dividirse en un número de nodos lo suficientemente grande (mallado del dominio). En cada uno de estos nodos, se aplicará la aproximación del modelo diferencial (discretización). Posteriormente, el sistema de ecuaciones obtenido se resuelve con el fin de hallar el valor de la variable dependiente en cada uno de los nodos generados. Es de esperarse que, a medida que se incrementa el número de nodos, la precisión de la solución obtenida será mayor, pero en la mayoría de los casos, la convergencia es más lenta (Jiménez-Islas, 1999).

Por otra parte, el método de colocación ortogonal pertenece a la familia de métodos conocidos con el nombre de residuos ponderados. En estos métodos se propone una aproximación a la solución exacta, conocida como función prueba; pero se siguen manejando los operadores diferenciales originales. Esta aproximación generalmente es de tipo polinomial debido a su simplicidad y a sus propiedades en la derivación. La solución se puede expandir en una serie de funciones conocidas con coeficientes desconocidos. Estos coeficientes son calculados para satisfacer la ecuación diferencial de alguna manera. El criterio utilizado para seleccionar la manera de satisfacer la ecuación determina el método específico. El siguiente paso es formar el residuo y proponer una función de peso (o de ponderación) para la cual el residuo se aproxime a cero.

En comparaciones hechas entre el método de diferencias finitas y el de colocación ortogonal se observa que, para un número igual de nodos, el método de colocación ortogonal es el que presenta una solución más precisa y en un menor número de iteraciones (Finlayson, 1980). Sin embargo, existen en la ingeniería química, problemas complejos (interacción reacción-transporte) que presentan cambios bruscos en la topología de la solución para los cuales la aplicación del método de colocación ortogonal requiere de un número de nodos extremadamente grande o es inaplicable.

Una seria alternativa para la solución de EDP apuntando a la eliminación de las inestabilidades presentadas en las interacciones reacción-transporte es el empleo de formulaciones de ecuaciones integrales (FEI). Las FEI pretenden hacer la inversión analítica de los operadores diferenciales a operadores integrales empleando una integral de Kernel que también es llamada función de Green. La determinación de la función de Green del modelo anexa de manera exacta las condiciones de frontera (Greenberg, 1971; Haberman, 2003).

Debido al auge de los métodos convencionales de discretización en la solución de sistemas de EDP, el empleo de las funciones de Green en (FEI) ha sido poco abordado (ÁlvarezRamírez et al., 2007), y en particular el uso de la formulaciones de ecuaciones integrales para la solución de modelos de procesos en ingeniería química.

Las simulaciones numéricas en el control y optimización de procesos se ha vuelto cada vez más importantes, por lo que es necesario contar con métodos de solución numérica más precisos y que sean capaces de enfrentarse a las complejidades que se presentan en los modelos que describen iteraciones de reacción-transporte. Orientado a la eliminación de inestabilidades y eliminación de error de aproximación numérica que se pueden producir los métodos clásicos de discretización (CO y DF) se propone el uso de las FEIs. 


\subsection{Estado del arte}

Este trabajo aborda dos temas centrales: $i$ ) la solución numérica de modelos de reactores tubulares mediante el uso de formulaciones de ecuaciones integrales y ii) el control lineal retroalimentado de reactores tubulares. Por ello se hacen dos apartados del estado del arte haciendo referencia a la aplicación de las formulaciones de ecuaciones integrales en los procesos químicos y al control de reactores tubulares.

\subsubsection{Uso de formulaciones de ecuaciones integrales en procesos químicos}

A diferencia de los métodos clásicos de discretización (diferencias finitas y colocación ortogonal), la literatura reportada sobre el uso de Funciones de Green aplicadas en problemas donde interviene interacciones Reacción-transporte (como es el caso de los reactores tubulares), es pobre.

Las funciones de Green deben su nombre a los trabajos de George Green (1825), quien transformo los problemas con valores a la frontera en forma de ecuaciones diferenciales a ecuaciones integrales utilizando funciones Kernels de integración ahora llamadas Funciones de Green (Roach, 1982). Inicialmente esta técnica se aplico para resolver problemas de la electrostática, posteriormente gracias a los trabajos del físico Paul Dirac por medio de su conocida función delta, la técnica se extendió beneficiando a una gran gama de problemas en física y matemáticas.

Los primeros trabajos que aplican esta técnica son reportados por Kesten (1969), que mediante formulaciones de ecuaciones integrales evalúa el efecto de la difusión de calor y masa en la película sobre la velocidad de la reacción, en un catalizador poroso de geometría esférica, estimando los perfiles de concentración y temperatura internas de la partícula, estos resultados se comparan con un esquema clásico de diferencias finitas, determinando que con FEI es mas fácil de resolver numéricamente y los tiempos de computo son menores.

Dixit y Tavlarides (1982) realizaron el análisis de la reacción de sintesis Fisher-Tropsch mediante el cálculo del factor de efectividad $(\eta)$ a diferentes condiciones externas, para ello emplean los balances de masa y calor en forma diferencial que transforman a ecuaciones integrales. El análisis se hace para una partícula de catalizador poroso.

A mediados de los 80' Mukkavilli et al. (1987) realizaron el análisis de la reacción química no isotérmica de un catalizador poroso con forma cilíndrica considerando un modelo en dos dimensiones (coordenadas radial y axial), para diferentes condiciones de frontera (con y sin resistencia externa). Emplean un esquema de Newton-Kantorovich para la solución de la ecuación integral no lineal resultante de FEIs.

Onyejekwe $(1996 ; 2002)$, mediante el uso de formulaciones de ecuaciones integrales determina la solución numérica de sistemas de ecuaciones reacción-difusión en diferentes geometrías de catalizador (rectangular, cilíndrica y esférica), también emplea un modelo de reacción-difusión de un proceso donde se lleva a cabo una reacción de combustión en un reactor tubular. Propone un método de elemento de Green.

Recientemente Valdés-Parada et al. (2007), con base en extensas simulaciones demuestran que en procesos tanto de reacción-difusión-convección como en reacción-difusión (hacen la 
variación de las expresiones velocidad de reacción y los parámetros constantes que rigen el modelo, para ver el efecto a diferentes condiciones) las FEIs tienen grandes ventajas frente a esquemas clásicos de diferencias finitas, que se reflejan en la reducción del tamaño de mallado en la discretización y en el tiempo de computo. El uso de operadores integrales en vez de operadores diferenciales disminuye y suaviza los errores de aproximación numérica además que tienen la gran ventaja que las condiciones de frontera son incorporadas de manera exacta.

\subsubsection{Control de reactores tubulares}

A diferencia del reactor continuo de tanque agitado (CSTR), la literatura reportada sobre control de reactores tubulares es escasa, sin embargo han existido esfuerzos notables por controlar este tipo de sistemas. Los esquemas de control para reactores tubulares se basan en técnicas de control lineal y no lineal, aquí mencionaremos algunos de los más representativos.

Strangeland y Foss (1970) diseñaron un esquema de control para un modelo de reactor flujo tapón hẹterogéneo adiabático mediante técnicas de control lineal. El control se basó en las ecuaciones linealizadas alrededor del estado estacionario deseado, obteniendo la función de transferencia que relacionaba las desviaciones en composición y temperatura con las condiciones de entrada del reactor. Además, investigaron la inyección de una corriente de alimentación secundaria y el efecto de su temperatura para controlar el estado de salida del reactor.

Georgakis et al. (1977), diseñaron un esquema de control modal para el caso de un reactor tubular con dispersión axial enchaquetado donde ocurre una reacción exotérmica irreversible. El desarrollo del control consistió en diseñar la chaqueta de enfriamiento para implementar la acción de control, discretizar las ecuaciones diferenciales parciales del modelo a través de colocación ortogonal, y diseñar el esquema de control basado en la descomposición modal del sistema. Además, por medio del observador de Luenberger reconstruyeron el perfil de concentración del reactor con mediciones de temperatura, para el caso donde concentración no era posible medirse. El esquema de control se implementó para estabilizar el estado inestable del reactor.

Wallman et al. (1979) diseñaron un sistema de control lineal multivariable para regular la concentración de producto de un reactor catalítico de lecho fijo. El sistema está compuesto de un estimador de la concentración de producto y un controlador PI multivariable que ajusta la velocidad de flujo y la temperatura de una corriente de apagado. En este caso se utilizan reconstructores de perturbaciones y de estados para implementar la ley de control resultante.

A principios de los 80 , Foss et al. (1980) re-consideran el reactor tubular y desarrollaron un sistema de control multivariable de tres entradas para la regulación de la concentración del producto y la temperatura de un reactor catalítico exotérmico de dos lechos. Dos de las entradas son la velocidad de flujo y la temperatura de la corriente de apagado inyectada entre los dos lechos, la tercera entrada es la temperatura de alimentación. Se diseñó a través del método de localización de características un compensador dinámico en cascada, en este caso se consideró que es posible medir la concentración.

Dochain et al. (1990), diseñaron un esquema de control adaptivo para un reactor biológico de lecho empacado, basados sobre la discretización espacial por colocación ortogonal de las ecuaciones diferenciales parciales del modelo, controlando la concentración del sustrato a la 
salida mediante la velocidad de flujo. Patwardhan et al. (1992) aplicaron control predictivo no lineal a un reactor de lecho fijo adiabático con reacción irreversible y exotérmica. Para el desarrollo del algoritmo de control se discretizaron las ecuaciones del modelo por colocación ortogonal sobre elementos finitos.

Hanczyc y Palazoglu (1995), desarrollaron un esquema de control de modo deslizante (Sliding mode control) para el caso de un reactor tubular no isotérmico. La idea principal del control de modo deslizante es definir una superficie sobre la cual el sistema tiene un comportamiento deseado. Para conducir las trayectorias del sistema a la superficie deslizante se proponen leyes de control basadas en condiciones de estabilidad de Lyapunov. Debido a que el modelo del sistema está descrito por ecuaciones diferenciales parciales del tipo hiperbólico, es posible utilizar el método de características para discretizar las ecuaciones y extender la teoría del control de modo deslizante para sistemas de parámetros distribuidos. Para la implementación de la ley de control fueron necesarias 5 mediciones de temperatura a lo largo del reactor.

Christofides (1995) propuso una metodología para sintetizar leyes de control robusto retroalimentado de sistemas de EDPs parabólicas cuasi-lineales con parámetros inciertos y dependientes del tiempo (modelos de reactores tubulares), y para los cuales su eigenespectro del operador diferencial espacial puede ser representado como una suma de dos sistemas, uno finito dimensional lentamente estable y otro infinito dimensional rápidamente estable, en 2001 determino la ubicación optima de los actuadores para el mismo problema.

Hua y Jutan (2000) desarrollaron un esquema de control no lineal inferencial en cascada para reactores tubulares con lecho empacado, el objetivo del control es mantener fija la concentración a la salida del reactor mediante la manipulación de la temperatura de la camisa que es manipulada por los requerimientos de la temperatura del reactor. Se plantea una discretización mediante colocación ortogonal.

Mediante técnicas de control lineal Alvarez-Ramirez et. al. (2001) estudian la regulación de la concentración del sustrato a la salida de un bio-reactor tubular de tratamiento de aguas residuales mediante un controlador retroalimentado en la frontera. La estructura del controlador se basa en modelos de entrada-salida, la estructura del controlar es un compensador clásico PI.

El control basado en técnicas de backstepping, fue aplicado por Boskovic y Krstic (2002) para estabilizar globalmente la salida de un reactor tubular con dispersión axial. Costa et al. (2003), trabajaron con la solución numérica y optimización de sistemas de reactores de polimerización formuladas en (EDP) las que discretizan mediante colocación ortogonal. Wu et al. (2007), implementaron un modelo de control predictivo en un reactor tubular exotérmico mediante la reducción de orden del modelo con un esquema clásico de diferencias finitas (DF).

El problema de control en la composición en reactores tubulares se ha estudiado extensamente con esquemas de control lineal y no lineal. Uno de los problemas más fuertes en la implementación de esquemas de control en reactores tubulares es el retardo en la medición de composición, debido a la respuesta tardía de los dispositivos de medición. Debido al retardo en la medición de composición, un diseño de control con un lazo de retroalimentación basado únicamente en la medición de composición puede conducir a los controladores a un pobre desempeño e incluso inestabilidades. Para aliviar esta situación 
Urrea et al., (2008) proponen un esquema de control lineal PI (Proporcional-Integral) en cascada de composición- temperatura, el esquema de control en cascada se basa en la medición de la temperatura en un punto del reactor, debido a que el lazo de temperatura tiene una respuesta mas rápida que el lazo de composición el esquema de control tiene la característica de anticiparse a perturbaciones. Sin embargo, la respuesta del controlador en cascada frente a perturbaciones depende en gran medida de la localización del sensor de temperatura a lo largo del reactor, es decir, que si el sensor de temperatura esta localizado en el centro del reactor la respuesta del controlador frente a un cambio en la composición no será la misma si el sensor esta localizado en la entrada o salida del reactor, Urrea $e_{\text {t. }}$ al., proponen un esquema de control en cascada empleando para el lazo de temperatura una temperatura promedio ponderada de dos mediciones de temperatura localizadas en antes y después del hot spot para el caso de estudio abordado, este esquema de control en cascada mejora la respuesta del controlador frente a perturbaciones. En este trabajo exploramos el uso de tres sensores de temperatura distribuidos simétricamente a lo largo del reactor tubular, se analizan dos casos con diferente dinámica para cuantificar el comportamiento del controlador.

\subsection{Definición del Problema}

Actualmente la demanda de la calidad de los productos industriales ha ido en aumento, por lo que cada vez se requieren sistemas de optimización y control más robustos que cumplan con las demandas establecidas. La simulación dinámica para el diseño de algoritmos de control y optimización implica una gran reducción de costos en la producción a nivel industrial, por lo que es imprescindible contar con modelos matemáticos confiables que describan de manera mas precisa el comportamiento físico de los sistemas y al mismo tiempo contar con metodologías de solución numéricas estables que aminoren las incertidumbres de los modelos. Los métodos de discretización clásicos empleados en ingeniería son diferencias finitas y colocación ortogonal. Sin embargo, en sistemas con interacción reacción-transporte y en particular los modelos que describen el comportamiento de reactores tubulares, los métodos de discretización clásicos (DF y CO), no son estables en todo el rango de operación, además del error que se produce en la estimación numérica de los operadores diferenciales y la discretización de las condiciones de frontera. Esto nos lleva a limitaciones en el uso de dichos modelos, provocando incertidumbre en su análisis e implementación algoritmos de control. Debido a esta situación es necesario abordar alternativas en los métodos solución numérica que permitan eliminar lo más posible, errores de aproximación numérica e inestabilidades que se presentan en las interacciones reacción-transporte.

El problema de control en reactores tubulares es mantener la concentración a la salida del reactor en un valor constante deseado, manteniendo la temperatura del reactor debajo de los valores especificados. Al mismo tiempo se requieren de esquemas de control que cuenten con rápidas acciones de corrección frente a perturbaciones en la alimentación, por lo que es propuesto el control en cascada, sin embargo, el desempeño de los esquemas clásicos de control en cascada composición-temperatura frente a perturbaciones, depende en gran medida de la localización del sensor de temperatura, debido a que en diferentes localizaciones del sensor el controlador presenta diferentes tiempos de respuesta. Por ello se requieren técnicas de control en cascada que exploten las diferentes propiedades que proveen los sensores colocados en diferentes puntos del reactor. 


\subsection{Objetivos}

\subsubsection{Objetivo General}

Emplear formulación de ecuaciones integrales, para la solución numérica, análisis e implementación de algoritmos de control en cascada, en modelos de reactores químicos tubulares.

\subsubsection{Objetivos Particulares}

1. Resolver numéricamente modelos de reactores tubulares mediante FEIs y comparar con esquemas clásicos de discretización (DF y CO).

2. Determinar las propiedades del sistema que permitan la implementación de algoritmos de control en cascada.

3. Implementar al sistema esquemas de control en cascada con sensor de temperatura localizado en diferentes posiciones a lo largo del reactor tubular.

4. Implementar al sistema un esquema de control en cascada que explote la información provista por tres sensores de temperatura distribuidos simétricamente a lo largo del reactor tubular.

5. Determinar las respuestas de los controladores frente a perturbaciones en la alimentación y comparar el desempeño del controlador propuesto con el esquema clásico de control en cascada.

6. Verificar la robustez del controlador propuesto mediante la variación de los parámetros del sistema. 


\section{Capítulo 2}

\section{Formulaciones de}

\section{Ecuaciones Integrales}

En este capitulo se presenta una breve introducción acerca de las formulaciones de ecuaciones integrales (FEI) mediante Funciones de Green, partiendo de las propiedades de los operadores diferenciales, su importancia en las ecuaciones diferenciales y finalmente la obtención de la función de Green.

\subsection{Introducción}

Las ecuaciones diferenciales son importantes en ingeniería porque pueden ser una clara representación de un fenómeno real. La deducción de las ecuaciones diferenciales, uso e interpretación no es una tarea fácil, pero su buen uso e interpretación nos puede llevar a conclusiones acertadas y como consecuencia a una buena toma de decisiones.

Muchos de los modelos matemáticos que describen los fenómenos en ingeniería química están regidos bajo las ecuaciones diferenciales parciales (EDP), que tienen un rango mayor de aplicación en comparación a las ecuaciones diferenciales ordinarias. En todas las áreas de la ingeniería, es muy común trabajar y analizar estos modelos, así como de obtener la solución de estos problemas. En ingeniería química, los modelos que describen el comportamiento de los reactores tubulares, la destilación reactiva, intercambiadores de calor, separación de membranas etc. están formulados en base a ecuaciones diferenciales ya sean ordinarias (EDO) o parciales (EDP).

Existen diferentes métodos analíticos y numéricos para la solución de ecuaciones diferenciales ordinarias y parciales, en el presente trabajo abordaremos el método llamado formulaciones de ecuaciones integrales mediante funciones de Green. Para ello es necesario revisar a la teoría de los operadores diferenciales lineales, a su aplicación a las ecuaciones 
diferenciales y por último la obtención de la función de Green para la solución de una ecuación diferencial.

Se desarrollará con conceptos conocidos por los lectores y por algunos nuevos. Para hacerlo de fácil comprensión se hará con base en las ecuaciones diferenciales ordinarias de segundo orden con condiciones en la frontera y de coeficientes constantes, para que posteriormente al interesado en este concepto lo pueda generalizar a las ecuaciones diferenciales parciales.

\subsection{Operadores diferenciales lineales.}

La teoría básica de los operadores diferenciales son tomados de Greenberg, (1971). Sea $A(x)$ una función positiva, continua y derivable en $a<x<b ; B(x)$ y $C(x)$ son funciones continuas. Un operador diferencial de segundo orden se define como:

$$
L=A(x) \frac{d^{2}}{d x^{2}}+B(x) \frac{d}{d x}+C(x)
$$

Si $L$ se aplica a una función $u(x)$ continua y derivable en el mismo intervalo, se tiene

$$
L u(x)=A(x) \frac{d^{2}}{d x^{2}} u(x)+B(x) \frac{d}{d x} u(x)+C(x) u(x)=f(x)
$$

$L u(x)$ cumple con las propiedades de superposición y homogeneidad, esto es:

$$
\begin{gathered}
L[\propto u(x)+\beta u(x)]=\alpha L u(x)+\beta L u(x) \\
L[\propto u(x)]=\alpha L u(x)
\end{gathered}
$$

Para resolver la ecuación diferencial (2-2) de segundo orden se requieren condiciones en la frontera:

$$
\begin{aligned}
& u(a)=u_{a} \\
& u(b)=u_{h}
\end{aligned}
$$

Si realizamos y analizamos el producto interno entre dos funciones, es decir de $v(x)$ (continua y derivable en $a<x<b$ ) y $L u(x)$ e integramos:

$$
\int_{a}^{b} v(x) L u(x) d x=\int_{a}^{b} v(x)\left[A(x) \frac{d^{2}}{d x^{2}} u(x)+B(x) \frac{d}{d x} u(x)+C(x) u(x)\right] d x
$$

Reescribiendo la integral en el miembro derecho se tiene:

$$
\int_{a}^{b} v(x) A(x) \frac{d^{2}}{d x^{2}} u(x) d x+\int_{a}^{b} v(x) B(x) \frac{d}{d x} u(x) d x+\int_{a}^{b} v(x) C(x) u(x) d x
$$


Al resolver cada integral mediante integración por partes y denotando $u(x)=u, v(x)=v$, $A(x)=A, B(x)=B$ y $C(x)=C$ llegamos a:

$$
\int_{a}^{b} v L u d x=\int_{a}^{h} u(v A)^{\prime \prime} d x-\int_{a}^{b}(v B)^{\prime} u d x+\int_{a}^{b} v C u d x+\left[v A u^{\prime}-(v A)^{\prime} u+v B u\right]_{a}^{h}
$$

como

$$
\begin{aligned}
& (v A)^{\prime}=A^{\prime} v+v^{\prime} A \\
& (v B)^{\prime}=B^{\prime} v+v^{\prime} B
\end{aligned}
$$

entonces la ecuación (2-4) se puede expresar como:

$$
\int v L u d x=\int\left[u A^{\prime \prime}+\left(2 A^{\prime}-B\right) u v^{\prime}+\left(A^{\prime \prime}-B^{\prime}+C\right) v u\right] d x+\left[v A u^{\prime}-(v A)^{\prime} u+v B u\right]_{a}^{\prime}
$$

Factorizando $u$ y definiendo $L^{*} v=A \frac{d^{2} v}{d x^{2}}+\left(2 A^{\prime}-B\right) \frac{d v}{d x}+\left(A^{\prime \prime}+B+C\right) v$, se llega a la siguiente expresión:

$$
\int_{a}^{b} v L u d x=\int_{a}^{b} u L^{*} v d x+\left.J(u, v)\right|_{a} ^{b}
$$

que es el teorema de Green para una dimensión., donde $\left.J(u, v)\right|_{a} ^{b}=\left[v A u^{\prime}-(v A)^{\prime} u+v B u\right]_{a}^{b}$ es el conjugado y que depende de lo que ocurra en la frontera, para todo:

$$
\begin{array}{lll}
u(a)=u_{a} & \text { y } & v(a)=v_{a}^{\bullet} \\
u(b)=u_{b} & v(b)=v_{b}
\end{array}
$$

$L^{*}$ recibe el nombre de operador adjunto y si $L=L^{*}$, se dice que $L^{*}$ es un operador formalmente autoadjunto. $\mathrm{Si} J(u, v)=0$ implica que:

$$
\int_{a}^{b} v(x) L u(x) d x=\int_{a}^{b} u(x) L^{*} v(x) d x
$$

debiendo cumplir también:

$$
\begin{aligned}
& u(a)=v(a)=0 \\
& u(b)=v(b)=0
\end{aligned}
$$

entonces el problema se conoce como autoadjunto.

\subsubsection{Operador Sturm - Lióuville.}

Sea $L u(x)$ un operador diferencial lineal que se aplica a una función $u(x)$ en $a<x<b$. 


$$
L u(x)=A(x) \frac{d^{2}}{d x^{2}} u(x)+B(x) \frac{d}{d x} u(x)+C(x) u(x)=f(x)
$$

si se multiplica a la ecuación por $\frac{p(x)}{A(x)}$ y definiendo a $p(x)=\exp \left[\int \frac{B-A^{\prime}}{A} d x\right]$, $q(x)=\left[\frac{C(x)}{A(x)}\right] \rho(x)$ y $f(x)=\left[\frac{f(x)}{a(x)}\right] p(x)$ continuas, derivables y positivas. La ecuación $(2-2)$ se convierte en:

$$
p(x) \frac{d^{2} u(x)}{d x^{2}}+p^{\prime}(x) \frac{d u(x)}{d x}+q(x) u(x)=f(x)
$$

Con: $p^{\prime}(x)=B(x), p(x)=A(x)$ y $C(x)=q(x)$

Pudiendo expresarse (2-7) como:

$$
\frac{d}{d x}\left[p(x) \frac{d u(x)}{d x}\right]+q(x) u(x)=f(x)
$$

donde $L=$ recibe el nombre de operador Sturm - Liouville y que también es llamada forma autoadjunta, esto es para $L=L^{*}$.

Analizando el conjugado $\left.J(u, v)=\left[v A u^{\prime}-(v A)^{\prime} u-B v u\right)\right]_{a}^{b}$, para $p^{\prime}(x)=B(x)$ y $p(x)=A(x)$ tenemos: $\left.J(u, v)=\left[p v u^{\prime}-(v p)^{\prime} u-p^{\prime} v u\right)\right]_{a}^{b}=\left[p v u^{\prime}-v^{\prime} p u-p^{\prime} v u+p^{\prime} v u\right]_{a}^{b}$, donde $p=p(x)$, entonces finalmente el conjugado se representa como:

$$
J(u, v)=\left[p v u^{\prime}-v^{\prime} p u\right]_{a}^{b}=p\left[v u^{\prime}-u v^{\prime}\right]_{a}^{b}
$$

Por tanto la primera identidad de Green (Ecuación 2-5), para cuando el operador es Sturm - Liouville, es:

$$
\int_{a}^{b} v L u d x=\int_{a}^{b} u L^{*} v d x+p\left[v \frac{d u}{d x}-u \frac{d v}{d x}\right]_{a}^{b}
$$

o también:

$$
\int_{a}^{b}\left[v L u-u L^{*} v\right] d x=p\left[v \frac{d u}{d x}-u \frac{d v}{d x}\right]_{a}^{b}
$$

$Y$ si se deriva con respecto a $x$ en ambos lados se tiene que:

$$
v(x) L u-u(x) L * v(x)=\frac{d}{d x}\left[p\left[v \frac{d u}{d x}-u \frac{d v}{d x}\right]\right]_{a}^{b}
$$


que es conocida como la identidad de Lagrange. Todo esto nos lleva a un enunciado muy importante en las ecuaciones diferenciales de segundo orden: "toda ecuación de segundo orden puede ser convertida en un operador Sturm - Liouville".

\subsection{La función de Green}

Para resolver (2-10) definimos $v(x)=G\left(x, x^{\prime}\right)$, donde $G\left(x, x^{\prime}\right)$ es la función de Green, de manera que:

$$
\int_{a}^{b} G\left(x, x^{\prime}\right) L u d x=\int_{a}^{b} u L^{*} G\left(x, x^{\prime}\right) d x+J(u, G)
$$

La función de Green $G\left(x, x^{\prime}\right)$ es la respuesta en $x$ debido a la fuente concentrada en $x^{\prime}$ (Haberman, 2003), es decir

$$
L^{*} G\left(x, x^{\prime}\right)=\delta\left(x-x^{\prime}\right)
$$

con sus adecuadas condiciones de frontera. Sustituyendo (2-13) en (2-12)

$$
\int_{a}^{b} G\left(x, x^{\prime}\right) L u d x=\int_{a}^{b} u \delta\left(x-x^{\prime}\right) d x+J(u, G)
$$

Y por propiedades de la función impulso (ver apéndice A1) tenemos:

$$
\int_{a}^{b} u(x) \delta\left(x-x^{\prime}\right) d x=u\left(x^{\prime}\right)
$$

Por lo que sustituyendo (2-1.5) en (2-14) y despejando $u$ tenemos

$$
u\left(x^{\prime}\right)=-J(u, G)+\int_{a}^{b} G\left(x, x^{\prime}\right) L u d x
$$

Pero de (2-2) $L u(x)=f(x)$ y tomando en cuenta la propiedad de reciprocidad de la función de Green $G\left(x, x^{\prime}\right)=G\left(x^{\prime}, x\right)$ podemos invertir el argumento $x$ por $x^{\prime}$ por lo tanto:

$$
u(x)=-J(u, G)+\int_{a}^{b} G\left(x, x^{\prime}\right) f\left(x^{\prime}\right) d x^{\prime}
$$

La función $G\left(x, x^{y}\right)$ describen la influencia de una perturbación (impulso) concentrado en un valor $x^{\prime}$ sobre el valor de $u$ en $x$. Otra forma de visualizarlo es como sigue: Si interpretamos a $u(x)$ como un desplazamiento y a $f(x)$ como una fuerza por unidad de longitud, la expresión (2-17) muestra que $G\left(x, x^{\prime}\right)$ es el desplazamiento en $x$ debido a una fuerza de magnitud unitaria concentrada en $x$. 
2.3.1 Construcción de la función de Green.

Después de haber visto todo lo necesario para la solución de una ecuación diferencial, sólo nos queda por ver la obtención de la función de Green. Teniendo ya en consideración el principio de reciprocidad $\left(G(x ; x)=G\left(x, x^{\prime}\right)\right)$, la función de Green satisface (Greenberg, 1971):

$$
L G\left(x, x^{\prime}\right)=\delta\left(x-x^{\prime}\right)
$$

O bien:

$$
\frac{d}{d x}\left[p(x) \frac{d}{d x} G\left(x, x^{\prime}\right)\right]+q(x) G\left(x, x^{\prime}\right)=\delta\left(x-x^{\prime}\right)
$$

Analizando la singularidad $x=x^{3}$ e integrando con respecto a $x$ la expresión (2-18) para valores que van desde $a=x^{\prime}-\xi$ y $b=x^{\prime}+\xi$ :

$$
\int_{x^{\prime}-\xi}^{x^{\prime}+\xi} \frac{d}{d x}\left[p(x) \frac{d}{d x} G\left(x, x^{\prime}\right)\right] d x+\int_{x^{\prime}-\xi}^{x^{\prime}+\xi} q(x) G\left(x, x^{\prime}\right) d x=\int_{x^{\prime}-\xi^{\prime}}^{x^{\prime}+\xi} \delta\left(x-x^{\prime}\right) d x
$$

Al resolver las integrales y por propiedades de la función impulso:

$$
p(x) \frac{d}{d x} G(x, x)_{x^{\prime}-\xi}^{x^{\prime}+\xi}+\int_{x^{\prime}-\xi}^{x^{\prime}+\xi} q(x) G\left(x, x^{\prime}\right) d x=1
$$

Si $\xi \rightarrow 0$ implica que:

$$
\int_{x^{\prime}-\xi}^{x^{\prime}+\xi} q(x) G\left(x, x^{\prime}\right) d x=0
$$

entonces

$$
\left.p(x) \frac{d}{d x} G\left(x, x^{\prime}\right)\right|_{x^{\prime}-} ^{x^{\prime}+}=1
$$

Por lo que:

$$
G^{\prime}\left(x^{\prime^{+}}, x^{\prime}\right)-G^{\prime}\left(x^{\prime}, x^{\prime}\right)=\frac{1}{p(x)}
$$

Cuando: $G\left(x, x^{\prime}\right)=G\left(x^{\prime}, x\right)$, recordando que es para: $x=x^{\prime}$. Con base en todo esto ya es posible resolver una ecuación diferencial de segundo orden con condiciones en la frontera y de coeficientes constantes.

20 


\subsubsection{Método Alternativo para el cálculo de la función de Green}

El desarrollo expuesto en esta parte es tomado de Irvin y Mullineux, (1959). Por conveniencia consideraremos el operador $L=\frac{d}{d x}\left[p(x) \frac{d}{d x}\right]-q(x)$ autoadjunto multiplicado por la función $u(x)$

$$
\frac{d}{d x}\left[p(x) \frac{d u(x)}{d x}\right]-q(x) u(x)=f(x)
$$

El primer paso es la integración de (2-24) entre los limites $[a x], a=$ constante y $x=$ variable, usaremos la notación $u^{\prime}(a)=u_{a}^{\prime}, p(a)=p_{a}$. Entonces

$$
p(x) u^{\prime}(x)-\int_{a}^{x} q(\xi) u(\xi) d \xi=\int_{a}^{x} f(\xi) d \xi+c_{1}
$$

donde sobreponiendo $x=a$ tenemos que $c_{1}=p_{a} u_{a}^{\prime}$. Si multiplicamos a (2-25) por la integral definida de la función $v^{\prime}(x)$, entonces

$$
\int_{a}^{x} v^{\prime}(\xi) p(\xi) u^{\prime}(\xi) d \xi-\int_{a}^{x} v^{\prime}(\xi) \int_{a}^{\xi} q(\psi) u(\psi) d \psi d \xi=\int_{a}^{x} v^{\prime}(\xi) \int_{a}^{\xi} f(\psi) d \psi d \xi+c_{1} \int_{a}^{x} v^{\prime}(\xi) d \xi
$$

Integrando por partes a (2-26)

$$
\begin{aligned}
& {\left[p(\xi) v^{\prime}(\xi) u(\xi)\right]_{a}^{x}-\int_{a}^{x} u(\xi) \frac{d}{d \xi}\left\{p(\xi) \frac{d v}{d \xi}\right\} d \xi-v(x) \int_{a}^{x} q(\xi) u(\xi)+f(\xi) d \xi+} \\
& \int_{a}^{x} v(\xi) q(\xi) u(\xi) d \xi+\int_{a}^{x} v(\xi) f(\xi) d \xi=p_{a} u_{a}^{\prime}\left[v(x)-v_{a}\right]
\end{aligned}
$$

donde $v_{a}=v(a)$; de la ecuación (2-24)

$$
\frac{d}{d x}\left\{p \frac{d u}{d x}\right\}=q u+f(x)
$$

Por tanto (2-27) es (omitiendo los argumentos de las funciones)

$$
p v^{\prime} u-p_{a} v_{a}^{\prime} u_{a}-\int_{a}^{x} u(L v) d \xi-v p u^{\prime}+v p_{a} u_{a}^{\prime}+\int_{a}^{x} v f(\xi) d \xi=p_{a} u_{a}^{\prime}\left\{v-v_{a}\right\}
$$

Si $v$ se escoge como $L v=0$, i.e. $v$ es la solución de la ecuación homogénea

$$
\frac{d}{d x}\left(p v^{\prime}\right)-q v=0
$$


Con lo cual (2-29) se reduce a

$$
p v^{\prime} u-v p u^{\prime}+\int_{x_{1}}^{x} v f(\xi) d \xi+p_{1}\left[v u_{1}^{\prime}-v_{1}^{\prime} u_{1}\right]=0
$$

Similarmente la integración en otro punto $x=b$ y el uso de $w^{\prime}(x)$ en lugar de $v^{\prime}(x)$, donde $w^{\prime}(x)$ también satisface: $L w=0$. Empleando la nomenclatura de $w(b)=w_{b}, p(b)=p_{b}$, $u^{\prime}(b)=u_{b}^{\prime}$ y $u(b)=u_{b}$, tenemos

$$
p w^{\prime} u-w p u^{\prime}+\int_{x}^{b} w f(\xi) d \xi+p_{b}\left[w u_{b}^{\prime}-w_{b}^{\prime} u_{h}\right]=0
$$

Multiplicando (2-30) por $w(x)$ y (2-31) por $v(x)$ y haciendo la sustracción

$$
p u\left(v^{\prime} w-v w^{\prime}\right)+p_{a} w\left(v_{a} u_{a}^{\prime}-v_{a}^{\prime} u_{a}^{\prime}\right)-p_{b} w\left(v_{b} u_{b}^{\prime}-v_{b}^{\prime} u_{b}\right)+\int_{a}^{h} G(x, \xi) f(\xi) d \xi=0
$$

donde

$$
G(x, \xi)= \begin{cases}G_{1}(x, \xi)=v(\xi) w(x) & a \leq \xi \leq x \\ G_{2}(x, \xi)=v(x) w(\xi) & x \leq \xi \leq b\end{cases}
$$

$G(x, \xi)$ es la función de Green. La función $v$ y $w$ no son completamente especificadas por las condiciones $L v=0$ y $L w=0$. Para reducir la ecuación (2-32) requerimos que $v$ y $w$ deben satisfacer

$$
\begin{array}{llr}
L v=0 & \text { y } & \left(v_{a} u_{a}^{\prime}-v_{a}^{\prime} u_{a}\right) \equiv\left(v u^{\prime}-v^{\prime} u\right)_{x=a}=0 \\
L w=0 & \text { y } & \left(w_{b} u_{b}^{\prime}-w_{b}^{\prime} u_{b}\right) \equiv\left(w u^{\prime}-w^{\prime} u\right)_{x=b}=0
\end{array}
$$

Con la consideración (2-34) obtenemos la solución general de la ecuación diferencial (2-24) como

$$
u(x)=\frac{1}{p(x)\left[v(x) w^{\prime}(x)-v^{\prime}(x) w(x)\right]} \int_{a}^{b} G(x, \varepsilon) f(\xi) d \xi
$$

La ecuación (2-35) determina el perfil de $u(x)$ evaluando directamente las condiciones de frontera. 


\section{Capítulo 3}

\section{Estrategia de Control}

En este capitulo se describe brevemente. la importancia del control en los procesos quimicos, los antecedentes y las características del diseño de control en cascada a partir de técnicas de control lineal, el diseño de control en cascada aplicado a reactores tubulares y la descripción de la configuración propuesta al esquema de control en cascada empleando una temperatura promedio ponderada resultante de la medición de tres temperaturas a lo largo del reactor tubular

\subsection{Control de Procesos}

El control de procesos es una técnica que tiene una variedad de campos de aplicación pero su utilidad solo se observa a través de ser usada directamente en los procesos industriales. El control necesariamente interacciona con otras disciplinas para generar y aplicar tecnologías a situaciones reales que involucran problemas de calidad, seguridad y disposición de residuos.

En el área industrial se ha observado que la mejora de procesos no necesariamente proviene del hecho de usar nuevas tecnologías, si no más bien del diseño de técnicas de control y en ocasiones el conjunto entre ambos recursos. En la industria química actual se presentan objetivos que involucran mejoras de calidad en los productos, reducci6n de costos de producción, disminución de la contaminación, mayor flexibilidad de los procesos sujetos a cambios de mercado y de tecnología. De manera que el reto es lograr lo anterior contando con una industria ya cimentada que en algunas ocasiones tiene varios años de antigüedad. 
Bajo esta situación el control es capaz de cubrir estos aspectos al hacer un análisis propio de cada proceso y diseñar una técnica de control adecuada que cubra dichos objetivos. Si se diseña un buen control, lo que significa que se mantendrán los valores de referencia dados en las variables de control, a bajos márgenes de variación, entonces, se mejorara la calidad y se reducirán costos de producción.

\subsection{Control en Cascada}

El control en cascada es una de las herramientas más útiles para el diseño de controles avanzados. Su utilidad radica en su capacidad para rechazar perturbaciones y mejorar el desempeño de los controladores. Este tipo de estrategia es usada en los procesos químicos debido a que existen variables con medidas retardadas o procesos de gran capacitancia como las columnas de destilación. La implementación de una técnica de control convencional, como el uso de controladores con estructura proporcional e integral implica, retardos en las acciones de control y mal desempeño de los controladores, ya que las perturbaciones y los ruidos de medición no serán detectados al tiempo que ocurren y por lo tanto el control tardará en corregir la acción de control.

La idea de usar control en cascada se originó con el problema de rechazo de perturbaciones cuyos efectos se detectan más rápidamente en una salida de control secundaria que en la variable controlada. Entonces, el uso de control en cascada es recomendado para aquellos procesos en los cuales la dinámica de un lazo de control secundario es más rápida que la dinámica del proceso primario (Luyben, 1990). En estas condiciones la interacción entre los dos esquemas de control es prácticamente nula, debido a la diferencia en sus dinámicas y por la cual se dice que no se presentan problemas de sintonizado en el esquema de control en cascada.

En la Figura 3-1 se ilustra un diagrama de bloques correspondiente a un sistema de control en cascada. En lugar de ajustar el elemento de control final, por ejemplo una válvula reguladora, la salida del control primario es el punto de ajuste del control secundario.

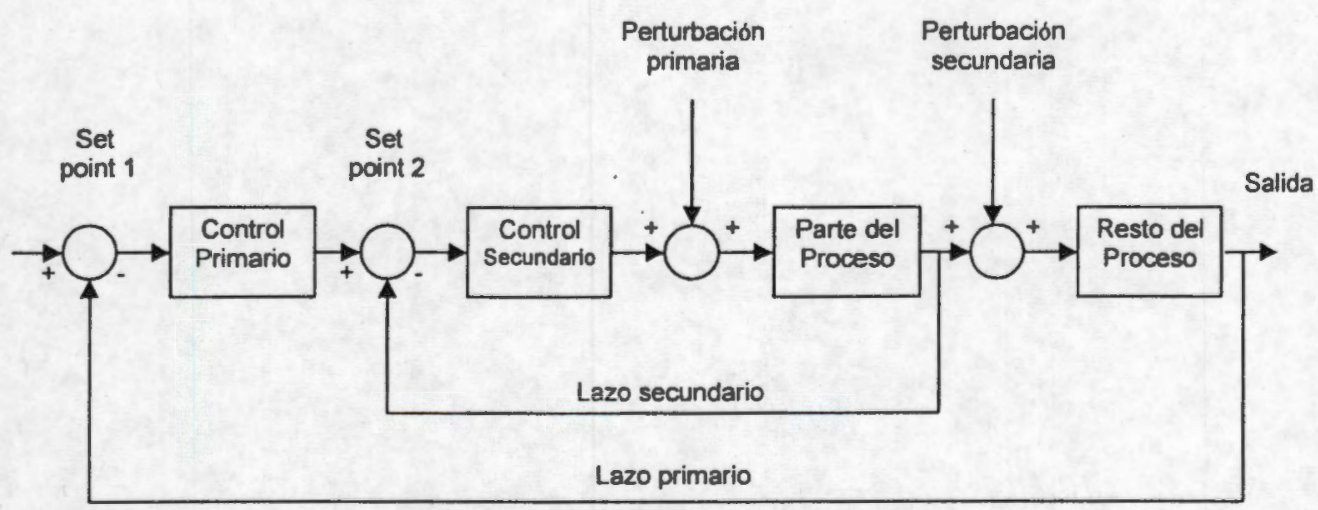

Figura 3-1 Diagrama de bloques de control en cascada

El lazo secundario abarca solo una parte del proceso total en un sistema de orden menor, de modo que el controlador se puede ajustar para dar una respuesta rápida. La eliminación de una fuente de perturbaciones hace disminuir el orden y las constantes de tiempo del proceso: 
estas reducciones aumentan la velocidad de respuesta que se puede obtener en un lazo de control primario. También reduce el tamaño de las variaciones en la variable controlada, mas allá de lo que seria posible incrementado la velocidad de respuesta del sistema de control primario.

\subsubsection{Antecedentes}

Yu, (1988), realizo trabajos de diseño y análisis de controladores tipo cascada paralelo. Mientras que Shen y Yu, (1990), aplicaron estos resultados para la selección de una variable medida secundaria cuando existen diferentes tipos de perturbaciones. Brambilla y Semino, (1992), introdujeron un filtro no lineal entre los dos lazos de control de un controlador cascada paralelo con el fin de desacoplarlos ya que la velocidad de respuesta de los dos esquemas era equiparable y no resultaba tan obvia la sintonización de los dos lazos de control. Brambilla, (1994) propuso la selección de algunos parámetros adimensionales para diseñar esquemas de control cascada paralelo en columnas de destilación con control dual. Semino y Brambilla, (1996) propusieron el diseño de una estructura de control cascada en paralelo capaz de evitar interacciones entre los lazos de control maestro y esclavo, utilizando un control convencional en el lazo interno y técnicas de modelo de control interno (IMC) en lazo externo con el fin de rechazar los efectos de las perturbaciones que no son tomadas en cuenta por el lazo interno, la estructura de control que ellos propusieron mostró mayores ventajas cuando la variable medida del lazo primario se obtiene con retardos. Russo y Bequette, (1997) construyeron una estructura de control cascada para el caso un RTAC de tres estados y usaron el modelo linealizado alrededor de un punto de equilibrio inestable, mientras que para la construcción del controlador utilizaron una estructura de IMC en el lazo externo o lazo esclavo para desacoplar a la temperatura del reactor de la temperatura del sistema de enfriamiento, y una estructura de un control convencional tipo proporcional en el lazo interno o maestro.

Alvarez-Ramirez et. al (2000), proponen un diseño de control en cascada adaptivo para sistemas no lineales con incertidumbres en las no linealidades, el esquema de control es basado sobre un diseño backstepping dotado de un estimador de error de modelado, donde para el caso de sistemas en una dimensión el controlador es equivalente a una ley de control PI, mientras que para sistemas de alta dimensionalidad puede ser interpretado como controladores PI tipo cascada.

Hua y Jutan (2000) desarrollaron un esquema de control no lineal inferencial en cascada para reactores tubulares con lecho empacado, el objetivo del control es mantener fija la concentración a la salida del reactor mediante la manipulación de la temperatura de la camisa que es manipulada por los requerimientos de la temperatura del reactor. Se plantea una discretización mediante colocación ortogonal.

Recientemente Urrea et. al. (2007), hacen una modificación al control en cascada concentración de destilado-temperatura para columnas de destilación, típicamente el lazo de temperatura emplea la medición de la temperatura en un plato en la zona de rectificación $\left(\mathrm{T}_{\mathrm{r}}\right)$ que es regulada por el reflujo de la columna. Se ha observado que la respuesta a perturbaciones en la temperatura de la zona de agotamiento $\left(T_{s}\right)$ de la columna es mas pronunciada que en la zona de rectificación. El esquema propuesto por los autores se basa en usar un promedio ponderado de las temperaturas $T_{s}$ y $T_{r}$, mejorando el controlador disminuyendo los efectos de las perturbaciones en el flujo de alimentación y la temperatura de 
entrada a la columna. Urrea et. al. (2008) proponen un esquema de control lineal PI (Proporcional-Integral) en cascada de composición- temperatura, el esquema de control en cascada se basa en la medición de la temperatura en un punto del reactor, debido a que el lazo de temperatura tiene una respuesta mas rápida que el lazo de composición el esquema de control tiene la característica de anticiparse a perturbaciones. Sin embargo, la respuesta del controlador en cascada frente a perturbaciones depende en gran medida de la localización del sensor de temperatura a lo largo del reactor, es decir, que si el sensor de temperatura esta localizado en el centro del reactor la respuesta del controlador frente a un cambio en la composición no será la misma si el sensor esta localizado en la entrada o salida del reactor, Urrea et. al., proponen un esquema de control en cascada empleando para el lazo de temperatura una temperatura promedio ponderada de dos mediciones de temperatura localizadas en antes y después del hot spot para el caso de estudio abordado, este esquema de control en cascada mejora la respuesta del controlador frente a perturbaciones. En este trabajo exploramos el uso de tres sensores de temperatura distribuidos simétricamente a lo largo del reactor tubular, se analizan dos casos con diferente dinámica para cuantificar el comportamiento del controlador.

El esquema de control empleado en este trabajo se basa en el propuesto por Urrea et. al (2008), analizando el efecto en el uso de tres sensores de temperatura para lazo de temperatura.

\subsubsection{Cuando usar el control en cascada}

El control en cascada es efectivo si el lazo interno es más rápido que el lazo externo, si la perturbación principal afecta primero al lazo interno. Normalmente un lazo de control en cascada no debería utilizarse si la constante de tiempo del lazo externo es por lo menos cuatro veces mayor que la constante de tiempo del lazo interno. (Smith y Corripio, 1980)

El control retroalimentado en su forma sencilla, provee un buen funcionamiento a lazo cerrado si la fracción de tiempo muerto es pequeña, perturbaciones son pequeñas y lentas, así como procesos con dinámica rápida, adicionalmente el segundo criterio requiere que la segunda variable pueda ser medida y agregue un costo aceptable o razonable.

Podemos decir entonces que los criterios para el diseño del control en cascada son:

1. Cuando el control retroalimentado simple no provee un desempeño satisfactorio a lazo cerrado.

2. La medición de la variable esta disponible.

La variable secundaria debe satisfacer los siguientes criterios:

1. Debe indicar la ocurrencia de una perturbación importante.

2. Debe haber una relación causal entre la variable manipulada y la segunda variable.

3. La variable secundaria debe tener una dinámica más rápida que la variable primaria.

Esto puede ser explicado de la siguiente manera:

Primero, debe poder indicar la ocurrencia de. una perturbación importante, esto es, la variable secundaria debe responder de una manera predecible cada vez que la perturbación 
ocurra. La perturbación debe tener un efecto significativo sobre la variable controlada y ocurrir frecuentemente, de otra manera, no hay razón para atenuar su efecto.

Segundo, la variable secundaria debe ser influenciada por la variable manipulada, la relación causal es requerida para que el lazo de control secundario trabaje apropiadamente. Finalmente la dinámica, entre el elemento final de control y la variable secundaria debe ser mucho más rápida que la dinámica entre la variable secundaria y la primera variable. La secundaria debe ser relativamente rápida de tal forma que pueda atenuar una perturbación antes que el efecto de la perturbación afecte la variable controlada.

\subsection{Sintonizado del controlador}

Es conocido que para el desempeño del control de un sistema de control en cascada depende fuertemente del sintonizado tanto del lazo primario como del secundario. Sin 'embargo la información publicada en la literatura sobre los métodos de sintonizado es limitada. Los métodos de respuesta de frecuencia (Hougen, 1979; Edgar et. al 1982) se usan comúnmente para el diseño de controladores porque la función de transferencia a lazo abierto del lazo primario tiene una dinámica de alto orden y/o tiempo muerto. Sin embargo, el mayor impedimento para usar el método de frecuencia para el diseño de controladores son los cálculos de gráficos que se obtienen prueba y error, los cuales pueden ser muy tediosos.

Krishnaswamy (1990), hace un estudio comparativo de control en cascada versus el control retroalimentado en sistemas una-entrada una-salida (SISO ${ }^{l}$ ), presentando un diseño de gráficos para determinar los parámetros de sintonizado del lazo primario. Sin embargo, estos resultados se ven limitados en configuraciones PI/P y a modelos de primer orden más tiempo muerto (MPOTM). Lee y Park (1988) proponen un método eficiente de sintonizado de controladores PID en cascada por medio de series de Maclaurin, este método puede ser aplicado a cualquier tipo de proceso estable a lazo abierto. Recientemente Skogestad (2003) presenta reglas analíticas para el sintonizado de controladores P/PI/PID, estas reglas son simples y tienen un buen comportamiento a lazo cerrado. Morari y Zafíriou, (1989) proporcionan una descripción breve del procedimiento de sintonizado de control PI/PID basado en modelos de control interno $\left(\mathrm{IMC}^{2}\right)$, estas reglas han sido empleadas para el diseño de controladores en cascada (Urrea, 2007 y 2008), teniendo buenos resultados en el desempeño del controlador.

\subsection{Control en cascada en reactores tubulares}

En el caso del control en la composición a la salida del reactor, un lazo de composición primario se combina con un controlador de temperatura secundario, el cual es conducido por la medición de la temperatura localizada en un punto lo suficientemente sensible a lo largo del reactor (Figura 3-2). Dado que los cálculos de la composición son generalmente tardados, debido a la limitación en la respuesta de los dispositivos de medición y al transporte interno, un lazo de retroalimentación basado únicamente en la medición de la composición puede presentar un pobre desempeño e incluso inestabilidades.

\footnotetext{
${ }^{1}$ Por sus siglas en ingles Single Input Single Output

${ }^{2}$ Por sus siglas en ingles Internal Model Control
} 
Para aliviar esta situación se ha propuesto el esquema de control en cascada composicióntemperatura. La idea básica es que la velocidad de flujo de alimentación o los efectos de perturbación en la composición puedan detectarse más rápidamente por el lazo de temperatura, para que pueden tomarse acciones correctivas anticipadas y neutralizar los efectos adversos.

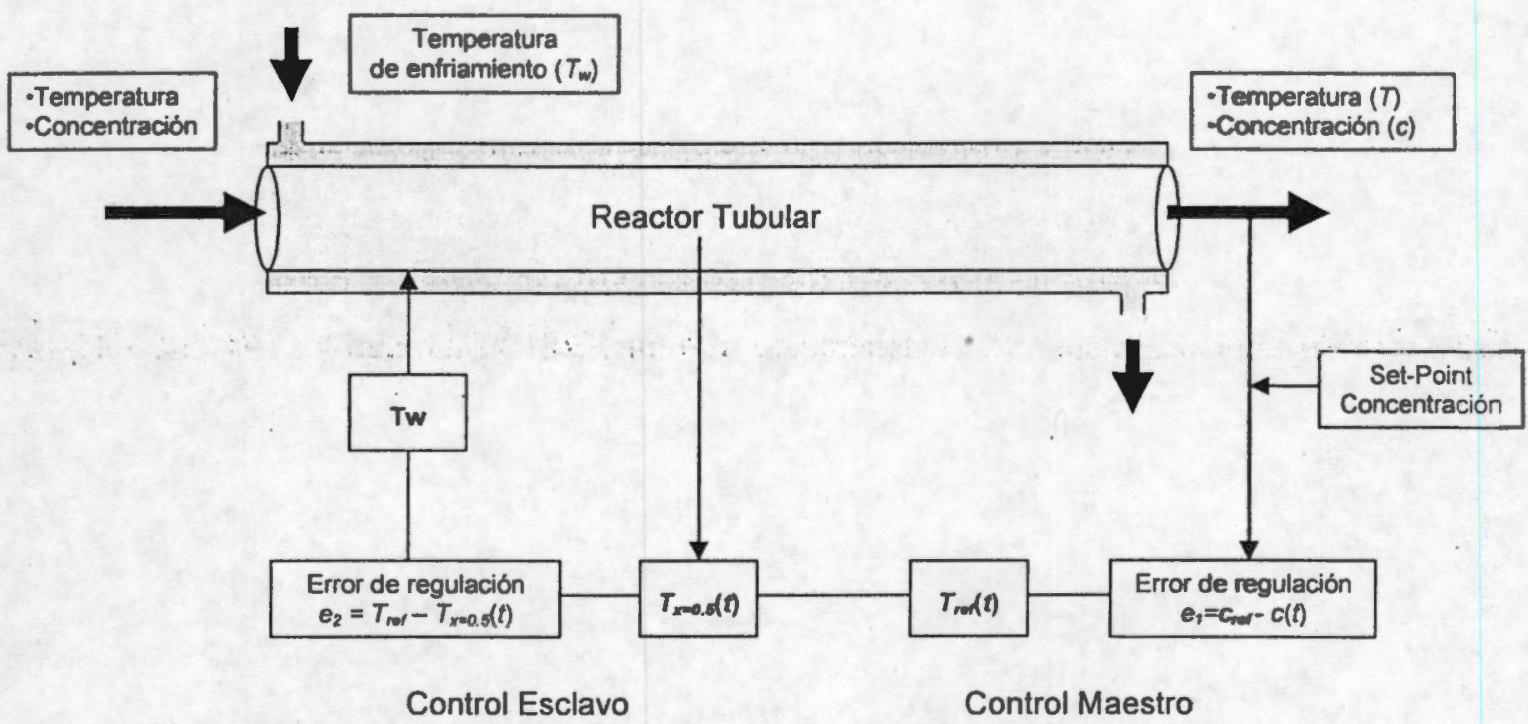

Figura 3-2 Representación del control en cascada del reactor tubular

\subsubsection{Diseño del controlador}

El control en cascada puede usar el control estándar retroalimentado P/PI/PID, lógicamente los tipo de controlador debe ser seleccionados para cada lazo del controlador. El controlador del lazo secundario debe tener el modo de control proporcional, pero no necesariamente requiere el modo integral, porque el objetivo completo del sistema de control es mantener la variable primaria en la referencia.

A pesar de esto, la parte integral se agrega frecuentemente por dos razones. La primera, ya que un controlador proporcional produce offset, el lazo secundario debería tener parte integral si se quiere eliminar por completo el efecto de la perturbación, evitando que la perturbación se propague al primario. En segundo lugar, la cascada se opera frecuentemente en forma parcial con el control primario fuera de operación, por ejemplo, cuando el sensor primario esta fuera de servicio o esta siendo calibrado. Sin embargo introducir el modo integral en el controlador secundario puede tender a comportamiento oscilatorio; el resultado puede ser que no sea significativo cuando el lazo secundario es mucho más rápido que el lazo primario. Los modos de control primario son obtenidos a partir del PI o PID, se debe enfatizar que el modo integral es esencial para garantizar cero error en condiciones estacionarias.

El esquema de control en cascada puede ser razonablemente manejado con diseños de control lineal convencional (IMC) y sensores de temperatura. Para el propósito del diseño de 
control en cascada, nos permitimos considerar modelos Entrada/Salida (E/S) de primer orden más tiempo muerto (MPOTM) calculados de una perturbación del $+5 \%$ en la temperatura de la pared $\left(T_{w}\right)$.

$$
\begin{aligned}
& \frac{c(s)}{T_{w}(s)}=G_{T_{w}}(s)=\frac{K_{T_{s}}}{\tau_{T_{w} s} s+1} \exp \left(-\theta_{T_{s} c} s\right) \\
& \frac{T_{i}(s)}{T_{w}(s)}=G_{T_{w} T_{i}}(s)=\frac{K_{T_{s} T_{i}}}{\tau_{T_{w i} T_{i}} s+1}
\end{aligned}
$$

Donde $\theta>0$ es el retraso debido a la medición y al transporte interno, $\tau_{T_{\text {s }}}$ y $\tau_{T_{\mathrm{T}} T_{i}}$ son las constantes de tiempo del proceso, $K_{T_{v}}$ y $K_{T_{v} T_{i}}$ son las ganancias del proceso, $c$ es la concentración a la salida del reactor y $T_{i}$ es la medición de la temperatura en una posición interna del reactor.

\subsubsection{Control en cascada convencional}

El controlador en cascada estándar de composición-temperatura esta compuesto por: (i) un lazo de composición, donde el lazo primario es manejado por la medición de la concentración y calcula los requerimientos de temperatura en un punto del reactor y (ii) un lazo de temperatura que manipula la temperatura de enfriamiento $\varpi$, llevando al set point la temperatura calculada en el lazo primario. Las reglas de sintonizado son de acuerdo a modelos de.control interno (IMC) (Morari y Zafiriou, 1989).

\subsubsection{Lazo de composición}

La función de transferencia que describe la relación entre la composición-temperatura puede ser descrito por el cociente de las Ecuaciones (3-1) y (3-2) tal que,

$$
\frac{c(s)}{T_{i}(s)}=\frac{G_{T_{s} c}(s)}{G_{T_{J} T_{i}}(s)}=G_{T_{i c}}(s)
$$

asi que

$$
G_{T_{r} c}(s)=K_{T_{s}}\left(\frac{\tau_{T_{s} T_{s}} s+1}{\tau_{T_{w}} s+1}\right) \exp \left(-\varepsilon_{T_{w} s} s\right)
$$

donde $K_{T_{i} c}=K_{T_{v} c} / K_{T_{w} T_{i}}$. Para condiciones cercanas al estado estacionario se puede aproximar $\frac{\tau_{T_{J_{1}}} s+1}{\tau_{T_{v} c} s+1} \approx 1$, lo que indica que la dinámica del retardo $\exp \left(-\varepsilon_{T_{v}} s\right)$ domina la dinámica global. Esto implica que

$$
G_{T_{i}}(s) \approx K_{T_{r}} \exp \left(-\varepsilon_{T_{w}} s\right)
$$

y en consecuencia, la regulación del lazo de composición se puede realizar con una retroalimentación integral simple, 


$$
C_{c T_{i}}(s)=\frac{K_{l, c}}{s}, K_{l, c}=\frac{1}{K_{T, c} \tau_{l, c}}
$$

con la ganancia integral $K_{l, c}$ ajustada de acuerdo a las reglas de sintonizado (IMC) (Morari y Zafiriou, 1989): $\tau_{l, c}$ alrededor de 1.5 a 2 veces el tiempo muerto $\varepsilon$.

\subsubsection{Lazo de temperatura}

El lazo secundario esta basado en (3-2). En procesos de primer orden un compensador PI es generalmente suficiente para alcanzar los objetivos de regulación (Morari y Zafiriou, 1989), y si el error de regulación esta dado por $e(s)=T_{i, \text { ref }}(s)-T_{i}(s)$ y la variable manipulada es $T_{w}$, el controlador PI correspondiente esta dado por

$$
C_{T_{i} T_{v}}(s)=K_{c, T_{i}}\left(I+\frac{1}{\tau_{l, T_{i}}}\right), K_{c, J_{i}}=\frac{1}{K_{T_{w} T_{i}}} \frac{\tau_{T_{v} T_{i}}}{\tau_{T_{i}}^{*}}
$$

donde $\tau_{l, T_{i}}=\tau_{T_{s} T_{i}}$, con ganancia $K_{c, T_{t}}$ y tiempo integral $\tau_{c, T_{i}}$. De acuerdo a las reglas de sintonizado (Morari y Zafiriou, 1989): $\tau_{T_{i}}^{*}$ esta alrededor de 0.75 a 1.5 veces de $\tau_{T_{w} T_{i}}$.

\subsubsection{Diseño de control en cascada con temperatura promedio}

En un diseño de control en cascada convencional composición-temperatura en reactores tubulares, un lazo de temperatura con la medición de temperatura en una posición del reactor es usado para mantener la regulación de composición estable dados los retrasos por medición y transporte. Efectivamente, la operación segura es garantizada por la acción rápida de lazo de retroalimentación de temperatura (sin retrasos). Sin embargo, los retrasos intrínsicos que son debido al transporte a través del reactor pueden limitar el desempeño del esquema de control en cascada.

Se propone mejorar el esquema de control en cascada convencional para un reactor tubular que explote la información provista por dos sensores adicionales (industrialmente baratos) distribuidos a lo largo del reactor. El promedio ponderado de tres temperaturas (Ecuación 36), es considerado como una salida a ser regulada por un lazo secundario (SISO), con un set point de la temperatura promedio generado por un controlador de composición primario. De esa manera, la consideración de una salida de temperatura promedio produce una composición a temperatura estándar, el diseño de control en cascada con una temperatura promedio requiere de tres parámetros de ponderación y son parámetro de sintonizado adicional. El promedio ponderado de temperatura es

$$
T_{\alpha w}=\alpha T_{e}+\beta T_{c}+\gamma T_{s}
$$

donde $\alpha, \beta$ y $\gamma$ son parámetros constantes de ponderación a determinar, la suma de los parámetros de ponderación debe ser la unidad $(\alpha+\beta+\gamma=1)$. El lazo de temperatura es basado en un modelo de primer orden, empleando ahora un promedio de temperaturas 


$$
\frac{T_{o v}(s)}{T_{w}(s)}=G_{T_{w} T_{m}}(s)=\frac{K_{T_{s} T_{w}}}{\tau_{T_{w} T_{w}}+1}
$$

En comparación con el control en cascada estándar de composición-temperatura, la propuesta del esquema con tres mediciones de temperatura debe tener una respuesta más rápida para el rechazo de perturbaciones a lazo cerrado, dado que un sensor de temperatura cercano a la entrada del reactor detecta rápidamente los cambios en las condiciones de entrada al reactor y un sensor de temperatura cercano a la salida del reactor se aproxima a las condiciones requeridas a la salida del reactor. 


\section{Capítulo 4}

\section{Simulaciones a}

\section{Lazo Abierto}

En este capitulo se presentan las simulaciones numéricas a lazo abierto de un reactor tubular con dispersión axial empleando Formulaciones de Ecuaciones Integrales. Los resultados numéricos son comparados con Diferencias Finitas y Calocación Ortogonal. Se consideran dos comportamientos diferentes del reactor, con y sin hot spot en el perfil de temperatura. Finalmente se analizó el comportamiento dinámico y estático del modelo a diferentes valores del numero de Pe.

\subsection{Reactor tubular con dispersión axial}

El modelo de flujo pistón puede sofisticarse al incluir dispersión axial y/o radial, para el transporte de masa y calor. El mezclado en la dirección axial, el cual se debe a la turbulencia o por la presencia del empaque, se toma en cuenta en un término de dispersión efectivo. El flux que surge por este mecanismo se describe por una fórmula análoga a la ley de Fick para la transferencia de masa y la ley de Fourier para la transferencia de calor. La dispersión axial se caracteriza por la relación del término difusivo y el término conductivo del transporte de calor y/o masa (i.e. los números de Peclet). Es una convención general que la dispersión axial de masa no es apreciable si la relación longitud del tubo sobre el diámetro del pellet no es mayor a 50, mientras para el calor es despreciable si esta relación es mayor a 300 (Aris, 1969). Existe evidencia experimental y teórica que indica que el coeficiente de dispersión axial es 10 veces mayor al coeficiente de dispersión radial, y es práctica común considerar solo la dispersión axial por simplicidad. Los modelos de dispersión axial se describen por un 
conjunto de ecuaciones diferenciales parciales parabólicas del tipo de valor en la frontera de dos puntos.

Las siguientes consideraciones son hechas en la derivación de las ecuaciones de balances de materia y energía sobre un elemento $x$ y $x+d x$ durante el intervalo $t a t+d t$.

1. Las variables dependientes son funciones solo dos variables independientes: $x$, longitud del reactor en el intervalo $0<x<l$ y $t$, el tiempo $>0$.

2. La velocidad promedio del flujo de reactantes es constante $v$

3. Los fluxes difusivos de masa y calor siguen las leyes de Fick y Fourier con una difusividad longitudinal efectiva $D_{m a}$ y una conductividad $k$.

4. La temperatura del reactor es controlada por la camisa a temperatura constante $T_{w}$ y el calor transferido de cualquier elemento es proporcional al valor local de $T-T_{w}$.

5. La velocidad de reacción $f(c, T)$ ha sido expresado en términos de la concentración, $c(x, t)$ y la temperatura $T(x, t)$.

\subsection{Modelo de reactor tubular}

En esta parte derivamos el modelo matemático del reactor tubular. El modelo del reactor no considera gradientes de concentración ni velocidad en dirección radial, el gradiente de temperatura es descrito por el uso apropiado del valor de la conductividad efectiva radial, se considera reacción homogénea, las propiedades de la reacción son caracterizadas por valores promedio, el mecanismo de mezclado axial es descrito por un solo parámetro del modelo y la velocidad de la reacción es considerada de primer orden. Las ecuaciones de balance de masa y energía para el reactor son tomados de Varma \& Aris, (1977).

$$
\begin{aligned}
& \frac{\partial c(x, t)}{\partial t}=D_{m a} \frac{\partial^{2} c(x, t)}{\partial x^{2}}-V \frac{\partial c(x, t)}{\partial x}-k_{o} c(x, t) e^{\left(-\frac{E}{R T}\right)} \\
& \frac{\partial T(x, t)}{\partial t}=\frac{\lambda_{e a}}{\rho C_{p}} \frac{\partial^{2} T(x, t)}{\partial x^{2}}-V \frac{\partial T(x, t)}{\partial x}-\frac{\Delta H}{\rho C_{p}} k_{p} c(x, t) e^{\left(\frac{E}{R T}\right)}-\frac{4 h}{\rho C_{p}}\left(T(x, t)-T_{w}\right)
\end{aligned}
$$

Con condiciones iniciales y de frontera

$$
\begin{aligned}
& c(x, 0)=c_{i n} \\
& T(x, 0)=T_{i n} \\
& D_{m a} \frac{d c(0, t)}{d x}=V\left(c(0, t)-c_{i m}\right) \\
& \frac{\lambda_{e m}}{\rho C p} \frac{d T(0, t)}{d x}=V\left(T(0, t)-T_{i n}\right) \\
& D_{m a} \frac{d c(L, t)}{d x}=0 \\
& \frac{\lambda_{e}}{\rho C p} \frac{d T(L, t)}{d x}=0
\end{aligned}
$$

En el sistema descrito por (4-1-4-8), $T$ denota la temperatura; $c$ representa la concentración; $V$, la velocidad lineal; $\lambda_{\text {ea }}$ el coeficiente de dispersión de energía en dirección 
axial; $D_{m a}$, el coeficiente de dispersión masico en dirección axial; $\Delta H$, el calor de reacción; $\rho$, la densidad del fluido; $C_{p}$ la capacidad calorífica; $k_{p}$, la constante cinética; $E$, la energía de activación; $R$, la constante de los gases ideales; $h$, coeficiente de transporte de calor de la pared del reactor; $d$, diámetro del reactor; $T_{w}$, la temperatura de enfriamiento; $T_{i m}$ temperatura de entrada al reactor y $c_{i n}$, denota la concentración del reactante a la entrada del reactor.

Introduciendo las variables adimensionales en posición, tiempo, concentración, temperatura y temperatura de enfriamiento como:

$$
\begin{aligned}
& \xi=\frac{z}{L}, \\
& \tau=\frac{V}{L} t, \\
& y(\xi, \tau)=\frac{c(x, t)-c_{\text {in }}}{c_{\text {in }}}, \\
& w(\xi, \tau)=\frac{E}{R T_{\text {in }}} \frac{T(x, t)-T_{\text {m }}}{T_{\text {in }}}, \\
& \varpi(\xi, \tau)=\frac{E}{R T_{\text {in }}} \frac{T_{w}(x, t)-T_{\text {in }}}{T_{\text {in }}},
\end{aligned}
$$

Obtenemos así un sistema adimensional.

$$
\begin{aligned}
& \frac{\partial y(\xi, \tau)}{\partial \tau}=\frac{1}{P e_{1}} \frac{\partial^{2} y(\xi, \tau)}{\partial \xi^{2}}-\frac{\partial y(\xi, \tau)}{\partial \xi}+D a(1-y(\xi, \tau)) e^{\left(\frac{w(\xi, \tau)}{1+w(\zeta, \tau) / \lambda}\right)} \\
& \frac{\partial w(\xi, \tau)}{\partial \tau}=\frac{1}{P e_{2}} \frac{\partial^{2} w(\xi, \tau)}{\partial \xi^{2}}-\frac{\partial w(\xi, \tau)}{\partial \xi}+B D a(1-y(\xi, \tau)) e^{\left(\frac{w(\xi, \tau)}{1+w(\xi, \tau) / \lambda}\right)}+\sigma(\varpi(\xi, \tau)-w(\xi, \tau))(
\end{aligned}
$$

Con condiciones iniciales y de frontera

$$
\begin{aligned}
& y(\xi, 0)=0 \\
& w(\xi, 0)=0 \\
& \frac{d y(0, \tau)}{d \xi}=P e_{1} y(0, \tau) \\
& \frac{d w(0, \tau)}{d \xi}=P e_{2} w(0, \tau) \\
& \frac{d y(1, \tau)}{d \xi}=0 \\
& \frac{d w(1, \tau)}{d \xi}=0
\end{aligned}
$$

donde $\mathrm{Pe}_{2}=\rho C p L V / \lambda_{\text {ea }}$, es el número de Peclet correspondiente al transporte de calor; $P e_{1}=L V / D_{m a}$, número de Peclet correspondiente al transporte de masa; $\sigma=(4 h / d)(L / \rho C p V)$, parámetro adimensional de transporte de calor; 
$D a=\left(k_{n} L / V\right) e^{-\left(E / R T_{m}\right)}$, número de Damköhler; $B=\left(-\Delta H C_{i n} / \rho C p T_{i n}\right)\left(E / R T_{i n}\right)$, temperatura adiabática adimensional; $\lambda=E / R T_{i m}$, es la energía de activación adimensional correspondiente.

Para la inversión del sistema de operadores diferenciales a operadores integrales mediante funciones de Green, analizaremos el sistema (4-14) y (4-15) en estado estacionario, por lo que las ecuaciones toman la forma de (4-22) y (4-23) con sus respectivas condiciones de frontera.

$$
\begin{aligned}
& \frac{1}{P e_{1}} \frac{\partial^{2} y(\xi, \tau)}{\partial \xi^{2}}-\frac{\partial y(\xi, \tau)}{\partial \xi}=-D a(1-y(\xi, \tau)) e^{\left(\frac{w(\xi, \tau)}{1+w(\xi, \tau) / \lambda}\right)} \\
& \frac{1}{P e_{2}} \frac{\partial^{2} w(\xi, \tau)}{\partial \xi^{2}}-\frac{\partial w(\xi, \tau)}{\partial \xi}=-B D a(1-y(\xi, \tau)) e^{\left(\frac{w(\xi, \tau)}{1+w(\xi, \tau) / \lambda}\right)}-\sigma(\varpi(\xi, \tau)-w(\xi, \tau))
\end{aligned}
$$

\subsection{Formulación del modelo en ecuaciones integrales}

La transformación de las ecuaciones se hace de manera particular para cada ecuación. Primero se hace la transformación del balance de masa (4-22) y en base a lo obtenido se hace la transformación del balance de energía (4-23). El operador de la ecuación correspondiente al balance de masa no es autoadjunto por lo que procedemos a hacer la transformación descrita en las ecuaciones (2-7) y (2-8) dando

$$
L=A(\xi) \frac{d^{2}}{d \xi^{2}}+B(\xi) \frac{d}{d \xi}+C(\xi)=\frac{1}{P e_{1}} \frac{d^{2}}{d \xi^{2}}-\frac{d}{d \xi}
$$

Por tanto

$$
A(\xi)=\frac{1}{P e_{1}} \quad B(\xi)=-1 \quad C(\xi)=0
$$

y

$$
\left.\sigma=\exp \mid \int-P e_{1} d \xi\right]=\exp \left(-P e_{1} \xi\right)
$$

Por comodidad denotaremos las funciones $y(\xi, \tau)=y$ y $w(\xi, \tau)=w$, si multiplicamos (4-25) por (4-22) tenemos:

$$
\exp \left(-P e_{1} \xi\right)\left[\frac{1}{P e_{1}} \frac{d^{2} y}{d \xi^{2}}-\frac{d y}{d \xi}\right]=\exp \left(-P e_{1} \xi\right) F(\xi, \dot{y}, w)
$$

o

$$
\frac{d}{d \xi}\left[\frac{\exp \left(-P e_{1} \xi\right)}{P e_{1}} \frac{d y}{d \xi}\right]=\phi(\xi, y, w)
$$

donde 


$$
\phi(\xi, y, w)=\exp \left(-P e_{1} \xi\right) F(\xi, y, w)=-\exp \left(-P e_{1} \xi\right) D a(1-y) e^{\left(\frac{w}{1+w / \lambda}\right)}
$$

Podemos reescribir la ecuación (4-26) como

$$
\frac{d}{d \xi}\left[\frac{\operatorname{Exp}\left(-P e_{1} \xi\right)}{P e_{1}} \frac{d y}{d \xi}\right]=\phi(\xi, y, w)
$$

que tiene como solución general la ecuación (2-35). Sustituyendo $P(x)=\frac{E x p\left(-P e_{1} x\right)}{P e_{1}}$, $f(x)=\phi(\xi, y, w) a=0$ y $b=1$ obtenemos

$$
y=\frac{1}{\frac{\operatorname{Exp}\left(P e_{1} \xi\right)}{P e_{1}}\left[u v^{\prime}-u^{\prime} v\right]_{0}^{1}} \int_{0}^{1} G(\xi, \psi) \phi(\xi, y, w) d \psi
$$

que satisface

$$
\frac{d}{d \xi}\left[\frac{\operatorname{Exp}\left(-P e_{1} \xi\right)}{P e_{1}} \frac{d u}{d \xi}\right]=0
$$

sujeta a la condición

$$
\left[u \frac{d y}{d \xi}-\frac{d u}{d \xi} y\right]_{\xi=0}=0
$$

y $v(\xi)$ es la solución de

$$
\frac{d}{d \xi}\left[\frac{\operatorname{Exp}\left(-P e_{1} \xi\right)}{P e_{1}} \frac{d v}{d \xi}\right]=0
$$

sujeta a la condición

$$
\left[v \frac{d y}{d \xi}-\frac{d v}{d \xi} y\right]_{\xi=0}=0
$$

Por lo tanto la función de Green esta dada por (2-33) con límites adimensionales de longitud del reactor en $a=0$ y $b=1$, y es expresada como:

$$
G_{m}(\xi, \psi)=\begin{array}{ll}
G_{1}(\psi, \xi)=u(\psi) v(\xi) & 0 \leq \psi \leq \xi \\
G_{2}(\psi, \xi)=u(\xi) v(\psi) & \xi \leq \psi \leq 1
\end{array}
$$

La función $u(\xi)$ es determinada por la integración de (4-29) 


$$
u=A_{1} \operatorname{Exp}(P e \xi)+B_{1}
$$

Aplicando la condición en la ecuación (4-30), $B_{l}=0$ asi $u(\xi)$ esta dada por

$$
u=A_{1} \operatorname{Exp}(P e \xi)
$$

y la función $v(\xi$ (es determinada por la integración de (4-31)

$$
v=A_{2} \operatorname{Exp}(P e x)+B_{2}
$$

Aplicando la condición de la ecuación (4-32), $A_{2}=0$ así $v(x)$ esta dada por

$$
v=B_{2}
$$

las Ecuaciones (4-28), (4-34) y (4-36) se combinan dando

$$
G_{m}(\xi, \psi)=\begin{array}{ll}
G_{1}(\xi, \psi)=A_{2} B_{2} \operatorname{Exp}(P e \xi) & 0 \leq \psi \leq \xi \\
G_{2}(\xi, \psi)=A_{2} B_{2} \operatorname{Exp}\left(P e_{1} \psi\right) & \xi \leq \psi \leq 1
\end{array}
$$

adicionando que

$$
\frac{1}{\frac{\operatorname{Exp}\left(-P e_{1} \xi\right)}{P e_{1}}\left[u v^{\prime}-u^{\prime} v\right]}=-A_{1} B_{2}
$$

Finalmente (4-37) se convierte en

$$
G_{m}(\xi, \psi)=\begin{array}{ll}
G_{1}(\xi, \psi)=-\operatorname{Exp}(P e \xi) & 0 \leq \psi \leq \xi \\
G_{2}(\xi, \psi)=-\operatorname{Exp}\left(P e_{1} \psi\right) & \xi \leq \psi \leq 1
\end{array}
$$

Sustituyendo.(4-39) en (4-28) tenemos la solución del balance de masa como:

$$
y=\int_{0}^{1} G_{m}(\xi, \psi) \phi(\psi, y, w) d \psi
$$

Dada la forma de las ecuaciones y las condiciones de frontera del balance de energía podemos expresar la solución general del perfil de temperaturas de manera análoga a la solución del perfil de concentraciones antes descrito, obteniendo así la siguiente expresión:

$$
w=\int_{0}^{1} G_{e}(\xi, \psi) \vartheta(\psi, y, w) d \psi
$$

donde $\vartheta(\psi, y, w)$ denota la velocidad de la reacción y èl transporte de calor a la camisa de enfriamiento y $G_{e}(\xi, \psi)$ es la función de Green correspondiente al balance de energía. Las 
ecuaciones (4-40) y (4-41) son la solución en estado estacionario del problema dado por las ecuaciones (4-22) y (4-23).

El problema dinámico se trata de la misma forma como se ha tratado el problema en estado estacionario, partimos transformado el operador de las ecuaciones (4-14) y (4-15) en un operador autoadjunto como se muestra en las ecuaciones (4-42) y (4-43)

$$
\begin{aligned}
& \exp \left(-P e_{i} \xi\right)\left[\frac{1}{P e_{1}} \frac{d^{2} y}{d \xi^{2}}-\frac{d y}{d \xi}\right]=\zeta(\xi, y, w) \\
& \exp \left(-P e_{2} \xi\right)\left[\frac{1}{P e_{2}} \frac{d^{2} w}{d \xi^{2}}-\frac{d w}{d \xi}\right]=\chi(\xi, y, w)
\end{aligned}
$$

donde

$$
\begin{aligned}
& \zeta(\xi, y, w)=\exp \left(\dot{\left.P e_{1} \xi\right)} \frac{d y}{d \tau}+\phi(\xi, y, w)\right. \\
& \chi(\xi, y, w)=\exp \left(P e_{2} \xi\right) \frac{d w}{d \tau}+\vartheta(\xi, y, w)
\end{aligned}
$$

Las expresiones (4-42) y (4-43) tienen la misma forma que la ecuación (4-28) por lo que su solución es análoga a las ecuaciones (4-40) y (4-41)

$$
\begin{aligned}
& y=\int_{0}^{1} G_{m}(\xi, \psi) \zeta(\psi, y, w) d \psi \\
& w=\int_{0}^{1} G_{e}(\xi, \psi) \chi(\psi, y, w) d \psi
\end{aligned}
$$

También podemos expresar a (4-46) y (4-47) como:

$$
\begin{aligned}
& y=\int_{0}^{1} \exp \left(-P e_{1} \psi\right) \frac{d y}{d \tau} G_{m}(\xi, \psi) d \psi+\int_{0}^{1} \phi(\psi, y, w) G_{m}(\xi, \psi) d \psi \\
& w=\int_{0}^{1} \exp \left(-P e_{2} \psi\right) \frac{d w}{d \tau} G_{e}(\xi, \psi) d \psi+\int_{0}^{1} \vartheta(\psi, y, w) G_{e}(\xi, \psi) d \psi
\end{aligned}
$$

\subsection{Consideraciones numéricas}

Una vez hecha la formulación de las ecuaciones en forma integral, tenemos que hacer la discretización de la integral para la solución numérica. Esto es mediante el uso de la regla de trapecio para aproximación numérica de la integral definida, al mismo tiempo que hacemos la discretización de los elementos en la dimensión $\xi$, como se muestra en las ecuaciones (4-49) 


$$
\begin{aligned}
& y\left(\xi_{1}\right)=\frac{h}{2}\left[G\left(\psi_{0}, \xi_{1}\right) \phi\left(\psi_{0}, y_{0}, w_{0}\right)+2 \sum_{i=1}^{n-1} G\left(\psi_{i}, \xi_{1}\right) \phi\left(\psi_{i}, y_{i}, w_{i}\right)+G\left(\psi_{n}, \xi_{1}\right) \phi\left(\psi_{n}, y_{n}, w_{n}\right)\right] \\
& y\left(\xi_{2}\right)=\frac{h}{2}\left[G\left(\psi_{0}, \xi_{2}\right) \phi\left(\psi_{0}, y_{0}, w_{0}\right)+2 \sum_{i=1}^{n-1} G\left(\psi_{i}, \xi_{2}\right) \phi\left(\psi_{i}, y_{i}, w_{i}\right)+G\left(\psi_{n}, \xi_{2}\right) \phi\left(\psi_{n}, y_{n}, w_{n}\right)\right] \\
& \vdots \\
& y\left(\xi_{n}\right)=\frac{h}{2}\left[G\left(\psi_{0}, \xi_{n}\right) \phi\left(\psi_{0}, y_{0}, w_{0}\right)+2 \sum_{i=1}^{n-1} G\left(\psi_{i}, \xi_{n}\right) \phi\left(\psi_{i}, y_{i}, w_{i}\right)+G\left(\psi_{n}, \xi_{n}\right) \phi\left(\psi_{n}, y_{n}, w_{n}\right)\right]
\end{aligned}
$$

El sistema de ecuaciones algebraico (4-49) puede resolverse mediante métodos numéricos de solución de sistemas de ecuaciones algebraicos no lineales tales como punto fijo o Newton Raphson multivariable. La solución antes descrita es para el caso donde el sistema esta en el estado estacionario.

Para el caso dinámico tenemos que agregar la parte de acumulación al balance de materia del reactor, empleando el mismo procedimiento presentado en las ecuaciones (4-49) tenemos un sistema de ecuaciones diferenciales ordinarias. Este sistema puede ser transformado en un sistema de ecuaciones algebraico y resuelto por métodos numéricos de solución de sistemas de ecuaciones algebraicos no lineales.

$$
\begin{aligned}
y\left(\xi_{1}\right)= & \frac{h}{2}\left[G\left(\psi_{0}, \xi_{1}\right) \frac{\partial y\left(\psi_{0}\right)}{\partial \tau}+2 \sum_{i=1}^{n-1} G\left(\psi_{i}, \xi_{1}\right) \frac{\partial y\left(\psi_{i}\right)}{\partial \tau}+G\left(\psi_{n}, \xi_{1}\right) \frac{\partial y\left(\psi_{n}\right)}{\partial \tau}\right]+ \\
& \frac{h}{2}\left[G\left(\psi_{0}, \xi_{1}\right) \phi\left(\psi_{0}, y_{0}, w_{0}\right)+2 \sum_{i=1}^{n-1} G\left(\psi_{i}, \xi_{1}\right) \phi\left(\psi_{i}, y_{i}, w_{i}\right)+G\left(\psi_{n}, \xi_{1}\right) \phi\left(\psi_{n}, y_{n}, w_{n}\right)\right] \\
y\left(\xi_{2}\right)= & \frac{h}{2}\left[G\left(\psi_{0}, \xi_{2}\right) \frac{\partial y\left(\psi_{0}\right)}{\partial \tau}+2 \sum_{i=1}^{n-1} G\left(\psi_{i}, \xi_{2}\right) \frac{\partial y\left(\psi_{i}\right)}{\partial \tau}+G\left(\psi_{n}, \xi_{2}\right) \frac{\partial y\left(\psi_{n}\right)}{\partial \tau}\right]+ \\
& \frac{h}{2}\left[G\left(\psi_{0}, \xi_{1}\right) \phi\left(\psi_{0}, y_{0}, w_{0}\right)+2 \sum_{i=1}^{n-1} G\left(\psi_{i}, \xi_{2}\right) \phi\left(\psi_{i}, y_{i}, w_{i}\right)+G\left(\psi_{n}, \xi_{2}\right) \phi\left(\psi_{n}, y_{n}, w_{n}\right)\right](4-5) \\
\vdots & \frac{h}{y\left(\xi_{n}\right)=} \frac{2}{2}\left[G\left(\psi_{0}, \xi_{n}\right) \frac{\partial y\left(\psi_{0}\right)}{\partial \tau}+2 \sum_{i=1}^{n-1} G\left(\psi_{1}, \xi_{n}\right) \frac{\partial y\left(\psi_{i}\right)}{\partial \tau}+G\left(\psi_{n}, \xi_{n}\right) \frac{\partial y\left(\psi_{n}\right)}{\partial \tau}\right]+ \\
& \frac{h}{2}\left[G\left(\psi_{0}, \xi_{1}\right) \phi\left(\psi_{0}, y_{0}, w_{0}\right)+2 \sum_{i=1}^{n-1} G\left(\psi_{i}, \xi_{n}\right) \phi\left(\psi_{i}, y_{i}, w_{i}\right)+G\left(\psi_{n}, \xi_{n}\right) \phi\left(\psi_{n}, y_{n}, w_{n}\right)\right]
\end{aligned}
$$

De la misma forma podemos expresar las ecuaciones para la temperatura tanto en estado estacionario como en el dinámico.

\subsection{Dinámica a lazo abierto}

El reactor tubular con dispersión axial descrito por las ecuaciones (4-14 - 4-21) y transformadas en ecuaciones integrales (4-41) y (4-42) presenta diferentes comportamientos de acuerdo a los valores de los parámetros empleados para su solución, dicho comportamiento 
es estable y con perfiles de temperatura y concentración cuasi-lineales (Varma y Aris, 1977), pero puede existir un perfil de temperatura estable con un punto caliente (hot spot) (Lefevre et al., 2000). Los dos casos anteriores se estudiaran a continuación:

\subsubsection{Perfil estable y cuasi-lineal}

Los parámetros que son empleados en el modelo se reportan en (Varma y Aris, 1977) y se presentan en la Tabla 4-1, el perfil de concentración se muestra en la Figura 4-1a-b y el perfil de temperatura en la Figura 4-1c-d a un tiempo adimensional de 20 unidades, los perfiles en estado estacionario se presentan en la Figura 4-1e-f. Las simulaciones están basadas en la discretización del sistema de ecuaciones integro-diferenciales (4-46) y (4-47) empleando un mallado de 100 nodos, es decir, se resolvieron un conjunto de 200 ecuaciones diferenciales simultáneamente.

Tabla 4-1 Parámetros para simulación del reactor tubular con dispersión axial.

\begin{tabular}{c|c} 
Parámetro & Valor \\
\hline$P e_{1}$ & 5.0 \\
\hline$P e_{2}$ & 5.0 \\
\hline$\sigma$ & 2.25 \\
\hline$B$ & 11.0 \\
\hline$D a$ & 0.174 \\
\hline$\lambda$ & 20.0 \\
\hline$\pi$ & 0.1
\end{tabular}
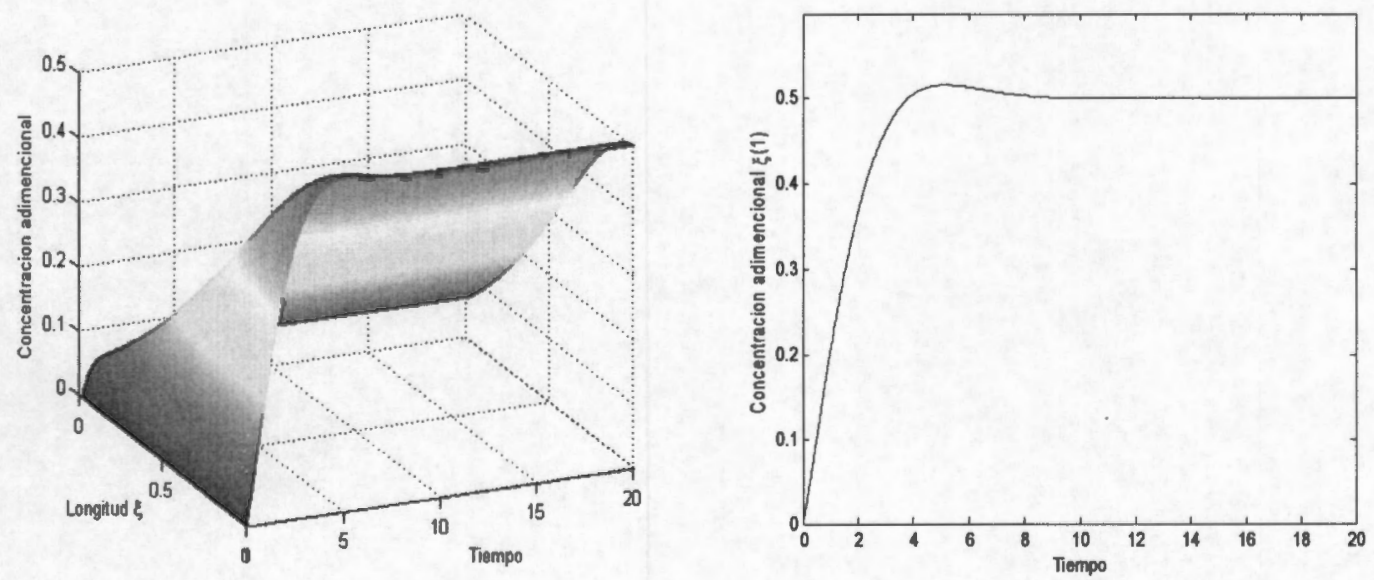

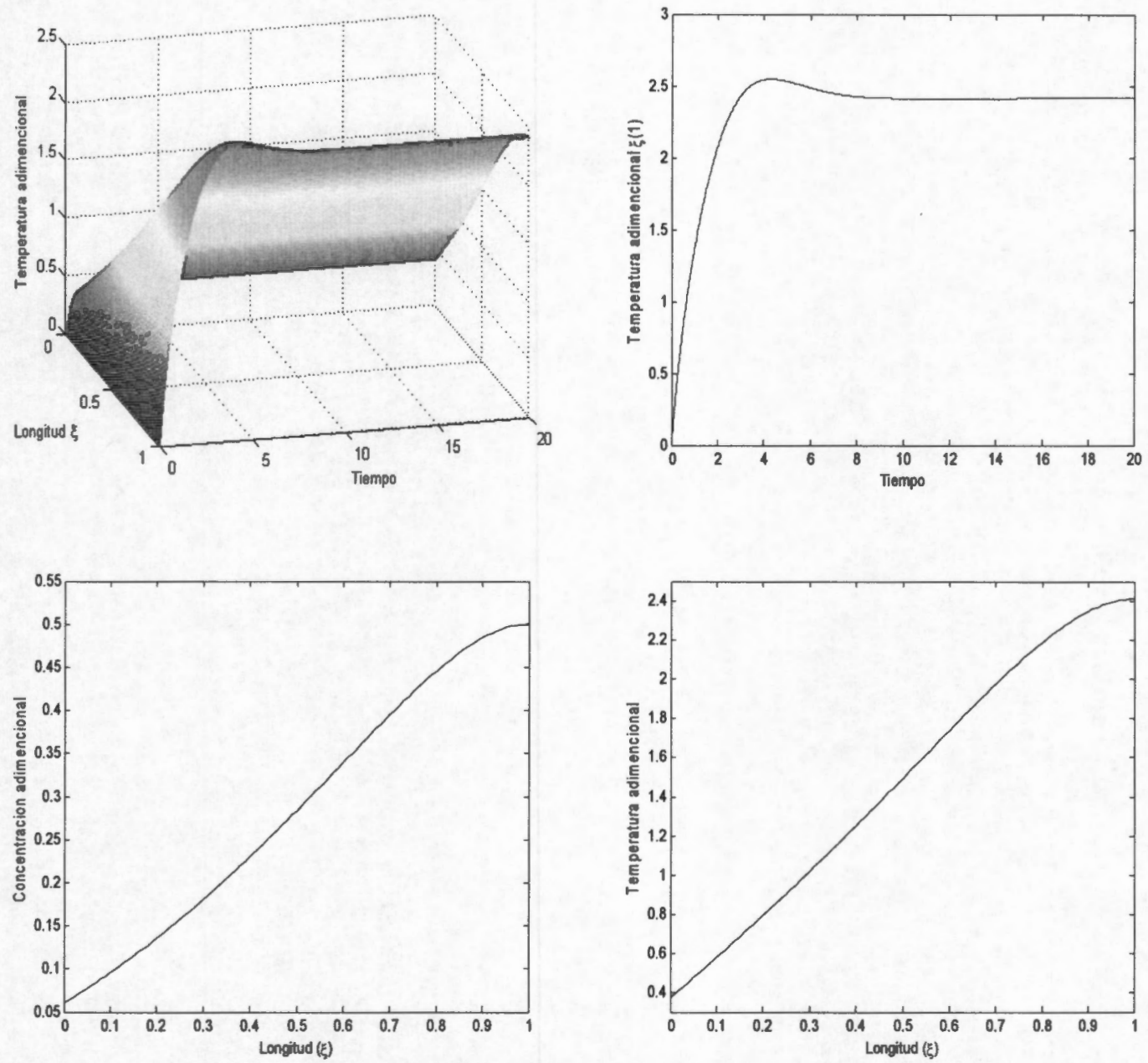

Figura 4-1 Perfiles de concentración y temperatura adimensional, a) perfil de concentración en 3D, b) concentración en $\xi=1$, c) perfil temperatura en 3D, d) temperatura adimensional en $\xi=1$, e) estado estacionario de concentración y f) de temperatura

\subsubsection{Perfil estable con hot spot}

Para este caso los parámetros son reportados por Lefevre y col., (2000) y se muestran en la Tabla 4-2. Las simulaciones se realizaron a las mismas condiciones que en la sección anterior. El perfil de concentración se muestra en la Figura 4-2a-b y el perfil de temperatura en la Figura 4-2c-d a un tiempo adimensional de 10 unidades, los perfiles en estado estacionario se presentan en la Figura 4-2e-f. 

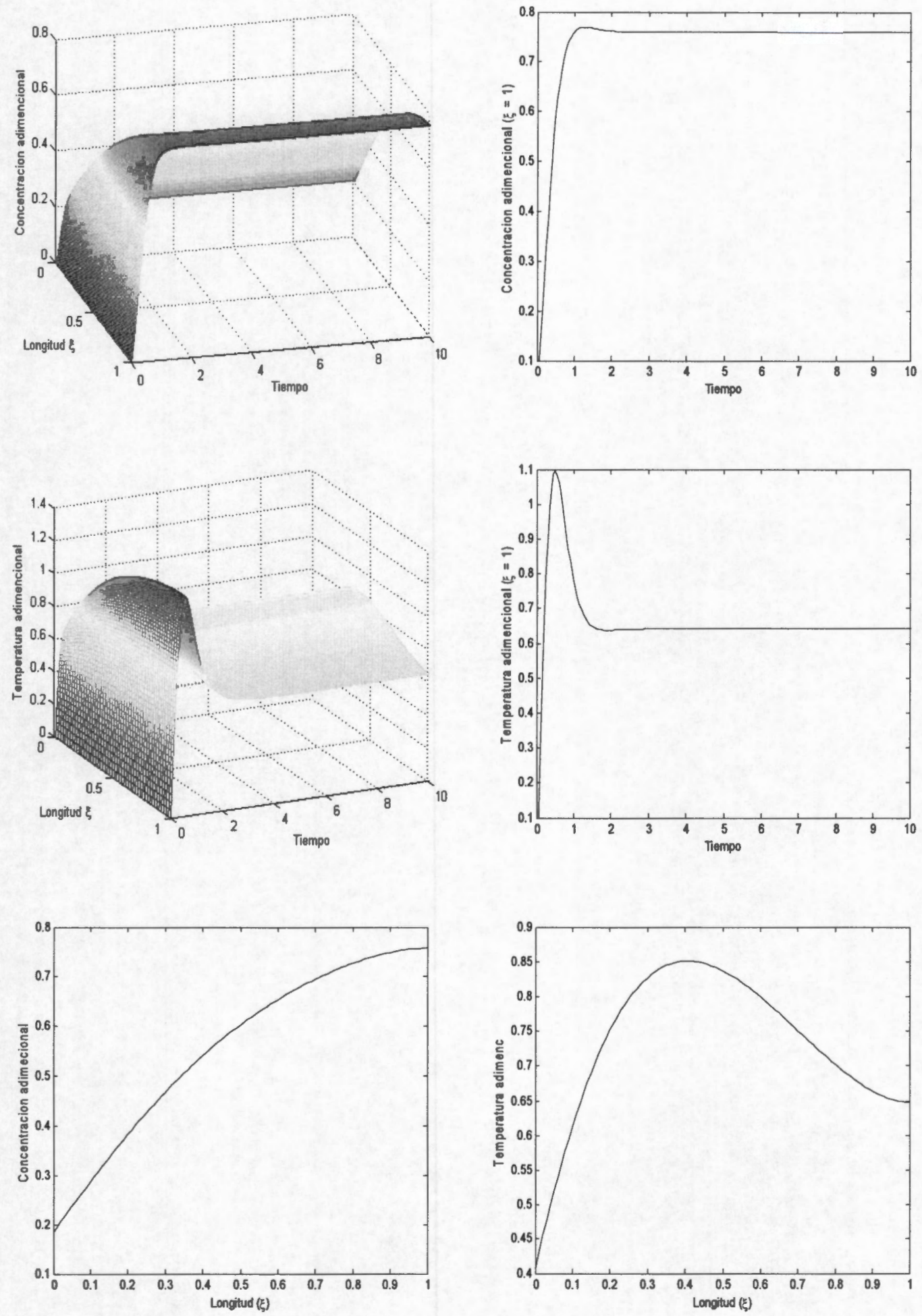

Figura 4-2 Perfiles de concentración y temperatura adimensional, a) perfil de concentración en $3 \mathrm{D}, \mathrm{b}$ ) concentración en $\xi=1, \mathrm{c}$ ) temperatura 3D, d) temperatura adimensional en $\xi=1$, e) estado estacionario de concentración y f) de temperatura 
El comportamiento cuando el perfil de temperaturas presenta un punto caliente se debe principalmente a los efectos de la velocidad de reacción, siendo una reacción exotérmica la que se lleva a cabo en el reactor, el punto más rápido de reacción y por tanto el punto donde se libera más calor es el hot spot.

Tabla 4-2 Parámetros para simulación del reactor tubular con perfil de temperatura con hot

\begin{tabular}{c|c} 
Parámetro & Spot. \\
\hline$P e_{l}$ & 5.0 \\
\hline$P e_{2}$ & 5.0 \\
\hline$\sigma$ & 5.0 \\
\hline$B$ & 5.0 \\
\hline$D a$ & 0.875 \\
\hline$\lambda$ & 20.0 \\
\hline$\varpi$ & 0.1
\end{tabular}

El tiempo de cómputo estimado es de $26.01 \mathrm{seg}$. para el perfil cuasi lineal y de $18.46 \mathrm{seg}$. para el perfil con hot spot. La simulación se lleva acabo en el lenguaje de programación Matlab $^{\circ}$ con el uso de un procesador intel celeron a $1.6 \mathrm{GHz}$. con $446 \mathrm{MB}$ de RAM.

\subsection{Análisis Numérico}

En esta sección se hace una comparación de los diferentes métodos, las FEI y los métodos clásicos de discretización CO y DF (ver apéndice $A 2$ y A3 respectivamente), identificando como marco de comparación el numero de nodos en la malla y el tiempo de computo. El análisis se hace para el estado dinámico y estacionario. Se realizaron pruebas de estabilidad al sistema haciendo variaciones en el número de Peclet, si $P e \rightarrow 0$ el proceso esta dominado por la difusión únicamente y si $P e \rightarrow \infty$ el proceso dominante es la convección.

\subsubsection{Análisis en estado estacionario}

La solución del sistema en estado estacionario para diferencias finitas y colocación ortogonal se hace a partir de las ecuaciones 4-22 y 4-23 y para la solución mediante ecuaciones integrales a partir de las ecuaciones $4-40$ y 4-41. Los sistemas algebraicos resultantes son resueltos mediante el método de punto fijo para solución de sistemas de ecuaciones algebraicos no lineales empleando para la convergencia un criterio de paro

$$
\frac{\sum_{i}^{N}\left|y_{i}-y_{i, b}\right|}{\sum_{i}^{N} y_{i}}<10^{-6} \text { y } \frac{\sum_{i}^{N}\left|w_{i}-w_{i, b}\right|}{\sum_{i}^{N} w_{i}}<10^{-6}
$$


donde $N$ es el numero de nodos empleados $y_{i}$ y $w_{i}$ son las concentraciones y temperaturas calculados en cada punto y $y_{i, b}, w_{i, b}$ son los valores anteriores respectivamente. Para fines de comparación se considero la solución exacta del sistema empleando diferencias finitas con 2000 nodos. Se calculo el error relativo como:

$$
\% \text { error }=\frac{\sum_{i}^{N}\left|w_{i}-w_{i, \text { exactoo }}\right|}{\sum_{i}^{N} w_{i, \text { exacto }}} \cdot 100
$$

\subsubsection{Perfil de temperatura cuasi lineal}

Los resultados presentados en esta sección son en base a los parámetros de la Tabla 4-1. El perfil de temperatura cuasi lineal a diferentes valores del número de Peclet se muestra en la Figura 4-3. En la Figura 4-3 observamos que a medida que el numero de Peclet es pequeño $\left(P e_{y}=P e_{w}=P e \rightarrow 0\right)$ la discontinuidad entre la temperatura adimensional entrante al reactor y la temperatura del reactor en la posición $\xi=0$ es mas grande, esto se debe al tipo de condiciones de frontera (Darckwert, 1953).

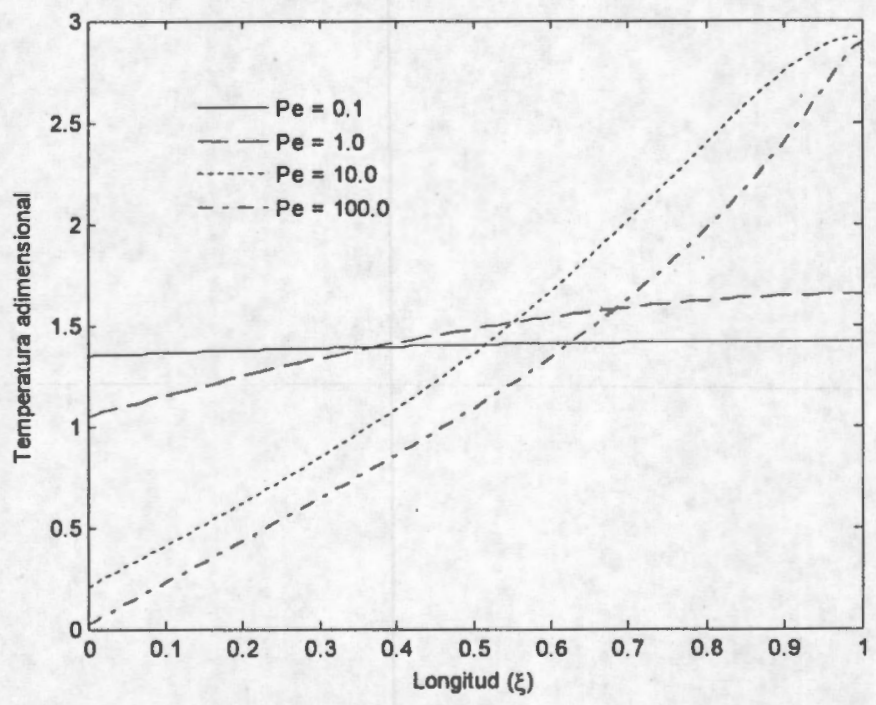

Figura 4-3 Perfil de temperatura cuasi lineal a diferentes números de Peclet en estado estacionario

La Figura 4-3 se obtuvo mediante la discretización de las Ecuaciones 4-22 y 4-23 mediante diferentes métodos DF, CO y FEI. Se calculo el error relativo (Ecuación 4-51) en cada caso variando el número de nodos en la discretización. Se determino que para el método de $\mathrm{CO}$ es suficiente usar un número máximo de 10 nodos para resolver el sistema, ya que el uso de un número mayor de nodos no cambia la aproximación numérica al de 10 nodos. La Figura 4-4 muestra el error relativo frente al número de nodos. El método de $\mathrm{CO}$ requiere un menor número de nodos que DF y FEI, además, el error relativo con numero de Peclet de $10^{1}$ es 
menor que DF y FEI incluso empleando 500 nodos en ambos métodos, pero a valores de Peclet de $10^{2}$ el método de CO muestra un error relativo mayor, tomando en cuenta el mayor numero de nodos de DF y FEI. Las FEI para todos los casos muestran menor error relativo que DF con la misma cantidad de nodos.
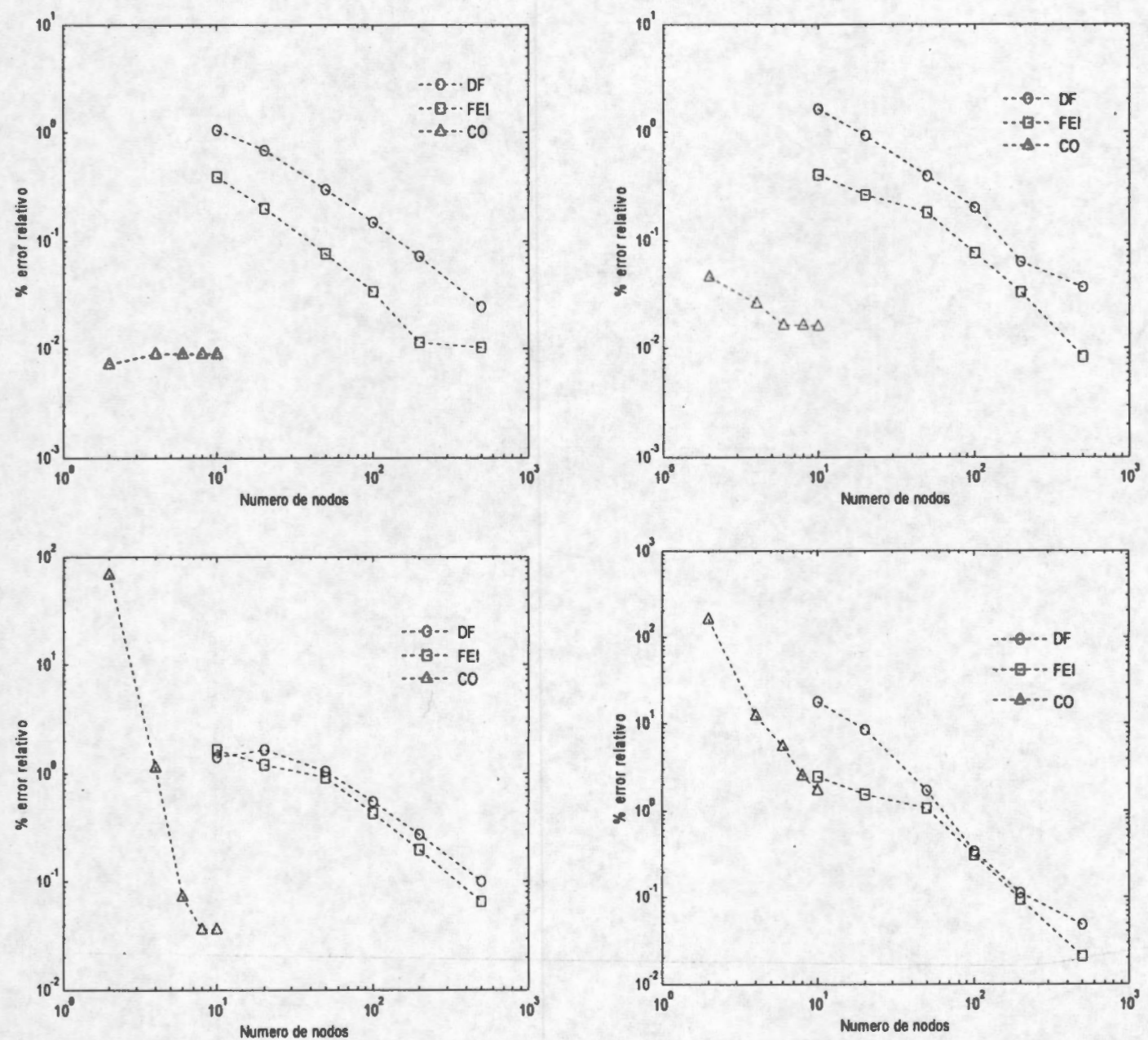

Figura 4-4 Aproximación numérica, caso: perfil cuasi lineal, error relativo vs. numero de nodos, a) $P e=0.1$, b) $P e=1.0$, c) $P e=10.0$ y d) $P e=100.0$

Otro parámetro para determinar la eficiencia numérica de los métodos de discretización antes descritos es el tiempo de cómputo. En la Tabla 4-3 se muestran los tiempos de cómputo empleados en la solución numérica del sistema para $\mathrm{CO}$, DF y FEI a valores de $\mathrm{Pe}=[0.1,1.0$, 10.0 y 100.0]. Debido a el reducido numero de nodos empleados en $\mathrm{CO}$, el tiempo de computo requerido para la solución del sistema es menor que el usado en DF y FEI. Sin embargo, podemos observar que el tiempo de cómputo empleado en la solución mediante FEI es menor que el empleado en DF usando el mismo numero de nodos y además cuando el $P e=$ $10^{2}$ los tiempos de computo son mejores incluso que los de $\mathrm{CO}$. 
Tabla 4-3 Tiempos de cómputo, perfil de temperatura cuasi-lineal

\begin{tabular}{|c|c|c|c|c|}
\hline \multicolumn{5}{|c|}{ Colocación Ortogonal } \\
\hline Nodos & \multicolumn{5}{|c|}{ Tiempo (s) } \\
\hline & $P e=0.1$ & $P e=1.0$ & $P e=10.0$ & $P e=100.0$ \\
\hline 2 & 1.18 & 1.20 & 1.27 & 1.24 \\
\hline 4 & 1.20 & 1.22 & 1.39 & 1.40 \\
\hline 6 & 1.35 & 1.39 & 1.43 & 1.44 \\
\hline 8 & 1.39 & 1.42 & 1.49 & 1.54 \\
\hline 10 & 1.45 & 1.50 & 1.56 & 1.67 \\
\hline
\end{tabular}

\begin{tabular}{|c|c|c|c|c|}
\hline \multicolumn{5}{|c|}{ Diferencias Finitas } \\
\hline Nodos & \multicolumn{5}{|c|}{ Tiempo (s) } \\
\hline & $P e=0.1$ & $P e=1.0$ & $P e=10.0$ & $P e=100.0$ \\
\hline 10 & 0.51 & 0.32 & 0.21 & 0.20 \\
\hline 20 & 1.04 & 0.45 & 0.42 & 0.37 \\
\hline 50 & 2.93 & 0.87 & 0.51 & 0.45 \\
\hline 100 & 18.28 & 4.46 & 0.90 & 0.85 \\
\hline 200 & 124.03 & 14.51 & 2.48 & 2.34 \\
\hline 500 & 1239.2 & 211.27 & 19.62 & 16.53 \\
\hline
\end{tabular}

\begin{tabular}{|c|c|c|c|c|}
\hline \multicolumn{5}{|c|}{ Formulaciones de Ecuaciones Integrales } \\
\hline Nodos & \multicolumn{5}{|c|}{ Tiempo (s) } \\
\hline & $P_{e}=0.1$ & $P e=1.0$ & $P e=10.0$ & $P e=100.0$ \\
\hline 10 & 0.23 & 0.11 & 0.12 & 0.10 \\
\hline 20 & 0.55 & 0.24 & 0.18 & 0.15 \\
\hline 50 & 1.24 & 0.70 & 0.69 & 0.58 \\
\hline 100 & 1.89 & 1.21 & 0.74 & 0.65 \\
\hline 200 & 4.65 & 2.11 & 1.15 & 0.84 \\
\hline 500 & 36.89 & 12.92 & 3.31 & 1.61 \\
\hline
\end{tabular}

Para los valores de $P e=[0.1,1.0,10.0]$, el método de $\mathrm{CO}$ requiere un menor número de nodos y tiempo de cómputo que el empleado para DF y FEI, esto para obtener semejantes errores relativos. Sin embargo para $P e=100.0$ el método de $C O$ requiere un mayor tiempo de computo que FEI, obteniendo un mayor error relativo, es decir, $\mathrm{CO}$ no puede resolver el sistema eficientemente en todo el rango de valores del numero de Peclet. Por otro lado DF y FEI pueden obtener menores errores relativos que $\mathrm{CO}$ en todo el rango de números de Peclet, pero empleando un gran numero de nodos y por consecuencia un mayor tiempo de cómputo. Es interesante observar que las FEI exhiben menores errores relativos y requieren menor tiempo de cómputo empleando el mismo número de nodos que DF.

\subsubsection{Perfil de temperatura con hot spot}

Para el análisis del sistema con perfil de temperatura con hot spot empleamos los mismos criterios mencionados en la sección anterior. Los resultados presentados son en base a los parámetros de la Tabla 4-2; resolvemos el sistema a diferentes valores del número de Peclet como se ve en la Figura 4-5. 


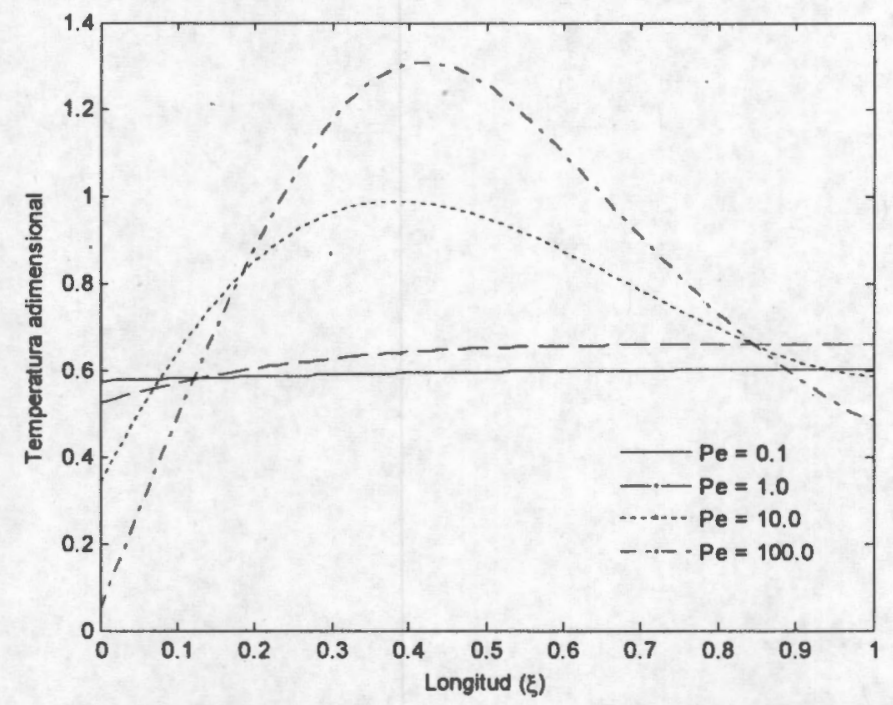

Figura 4-5 Perfil de temperatura con hot spot a diferentes números de Peclet en estado estacionario
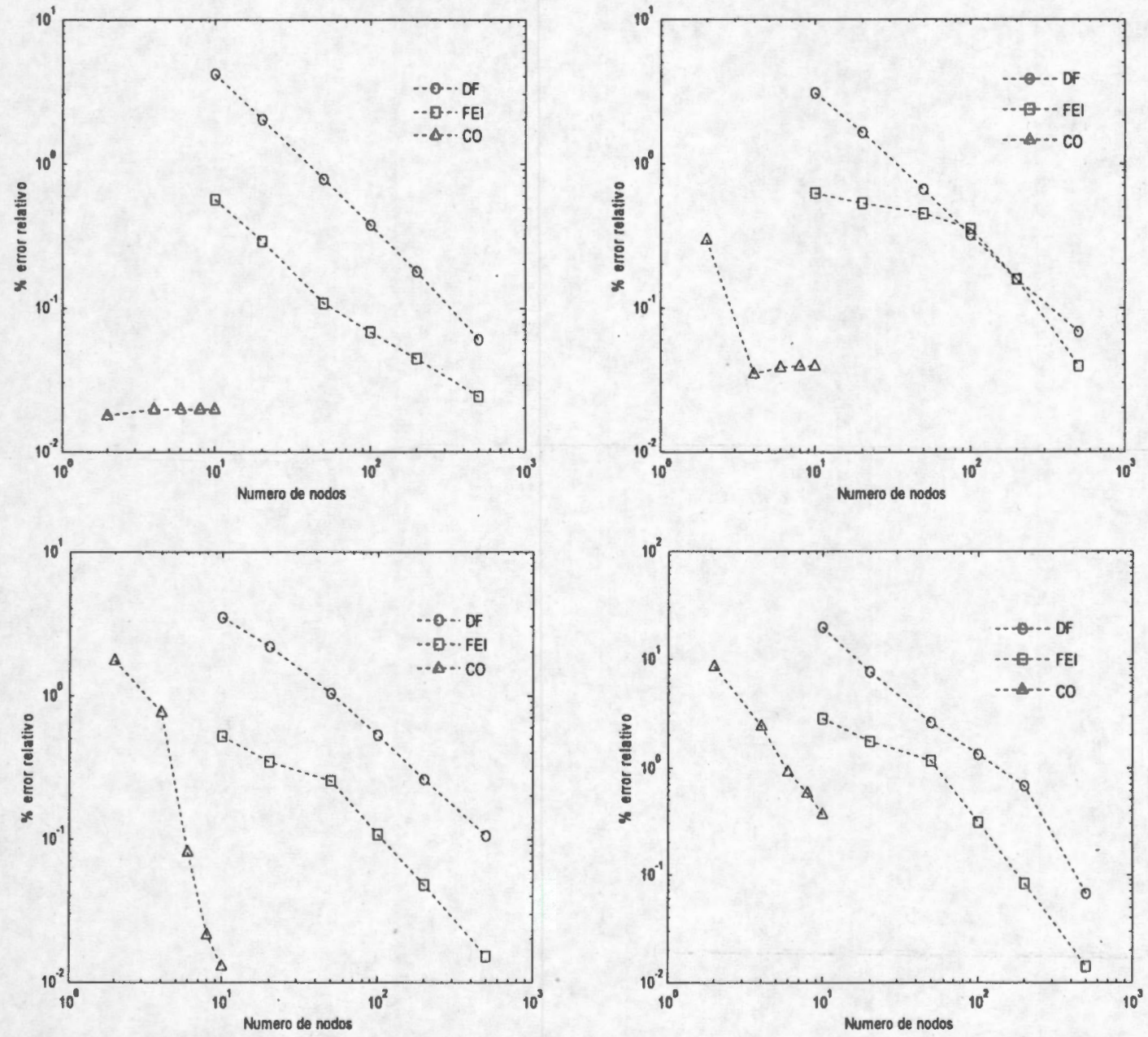

Figura 4-6 Aproximación numérica, caso: perfil con hot spot, error relativo vs. numero de nodos, a) $P e=0.1$, b) $P e=1.0$, c) $P e=10.0$ y d) $P e=100.0$ 
La Figura 4-6 muestra el error relativo frente el número de nodos empleados en la discretización para cada uno de los tres métodos empleados. Los resultados muestran la misma tendencia que los resultados de la sección anterior. El método de $\mathrm{CO}$ emplea por mucho un menor numero de nodos en la solución del sistema, sin embargo para valores del Pe $=10^{2}$ el sistema resuelto exhibe errores relativos mayores hasta en un orden de magnitud que DF y FEI. El método de las FEI a pesar de emplear un gran numero de nodos, es constante para todos los casos, es decir, muestra la misma tendencia del error relativo frente a el numero de nodos para los cuatro casos, además que el error relativo calculado es menor que el de DF con la misma cantidad de nodos empleados

Tabla 4-4 Tiempos de cómputo, perfil de temperatura con hot spot

\begin{tabular}{|c|c|c|c|c|}
\hline \multicolumn{5}{|c|}{ Colocación Ortogonal } \\
\hline Nodos & \multicolumn{5}{|c|}{ Tiempo (s) } \\
\hline & $\mathrm{Pe}=0.1$ & $\mathrm{Pe}=1.0$ & $\mathrm{Pe}=10.0$ & $\mathrm{Pe}=100.0$ \\
\hline 2 & 1.18 & 1.19 & 1.28 & 1.35 \\
\hline 4 & 1.23 & 1.23 & 1.43 & 1.46 \\
\hline 6 & 1.28 & 1.39 & 1.46 & 1.56 \\
\hline 8 & 1.35 & 1.47 & 1.51 & 1.59 \\
\hline 10 & 1.49 & 1.56 & 1.59 & 1.67 \\
\hline
\end{tabular}

\begin{tabular}{|c|c|c|c|c|}
\hline \multicolumn{5}{|c|}{ Diferencias Finitas } \\
\hline Nodos & \multicolumn{5}{|c|}{ Tiempo (s) } \\
\hline & $\mathrm{Pe}=0.1$ & $\mathrm{Pe}=1.0$ & $\mathrm{Pe}=10.0$ & $\mathrm{Pe}=100.0$ \\
\hline 10 & 0.51 & 0.28 & 0.15 & 0.12 \\
\hline 20 & 0.89 & 0.43 & 0.22 & 0.18 \\
\hline 50 & 1.44 & 0.71 & 0.32 & 0.30 \\
\hline 100 & 6.06 & 1.90 & 0.45 & 0.42 \\
\hline 200 & 43.72 & 11.20 & 1.72 & 1.53 \\
\hline 500 & 279.63 & 151.16 & 15.15 & 12.23 \\
\hline
\end{tabular}

\begin{tabular}{|c|c|c|c|c|}
\hline \multicolumn{5}{|c|}{ Formulaciones de Ecuaciones Integrales } \\
\hline Nodos & \multicolumn{5}{|c|}{ Tiempo (s) } \\
\hline & $\mathrm{Pe}=0.1$ & $\mathrm{Pe}=1.0$ & $\mathrm{Pe}=10.0$ & $\mathrm{Pe}=100.0$ \\
\hline 10 & 0.21 & 0.15 & 0.12 & 0.10 \\
\hline 20 & 0.34 & 0.23 & 0.18 & 0.14 \\
\hline 50 & 1.02 & 0.32 & 0.22 & 0.18 \\
\hline 100 & 1.54 & 0.74 & 0.51 & 0.43 \\
\hline 200 & 2.35 & 1.12 & 0.63 & 0.51 \\
\hline 500 & 16.96 & 4.20 & 2.05 & 0.89 \\
\hline
\end{tabular}

Los tiempos de cómputo estimados cuando el perfil de temperatura tiene hot spot para los tres métodos de solución son reportados en la Tabla 4-4. Como se menciono en la sección anterior los tiempos de cómputo para CO son generalmente menores que los empleados en DF y FEI para obtener el mismo error relativo, excepto en el caso cuando $P e=10^{2}$ los tiempos de computo de DF y FEI son menores para el mismo error relativo. De manera globlal los 
tiempos de computo de FEI son del orden de $\mathrm{CO}$ pero con la ventaja que las FEI son estables en todo el rango de valores del numero de Peclet.

\subsubsection{Análisis dinámico}

La solución del sistema en estado dinámico se hace a partir de las ecuaciones 4-14 y 4-15 que son las ecuaciones que describen el comportamiento del reactor en función del tiempo. En los apéndices $\mathrm{A} 2$ y $\mathrm{A} 3$ se da una breve descripción de discretización de las ecuaciones diferenciales con los métodos DF y $\mathrm{CO}$ respectivamente, mientras que para las $\mathrm{FEI}$ emplearemos las ecuaciones 4-48 y 4-49 que previamente transformamos en ecuaciones integrales. Para los sistemas de ecuaciones diferenciales se empleo el método de integración numérica Runge-Kutta-Dormand-Prince $(4,5)$ con paso de integración variable y para el sistema de ecuaciones integrales se empleo el método de integración numérica mediante la regla del trapecio.

\subsubsection{Perfil de temperatura cuasi lineal}

Para poder analizar la solución del sistema como función del tiempo, examinaremos la dinámica de la temperatura en la frontera $\xi=1$ a valores de Peclet como $(P e=[0.1,1.0,10.0$ 100.0]) en el caso estático. La Figura 4-7 muestra el perfil dinámico de la temperatura a diferentes valores $P \boldsymbol{e}$.

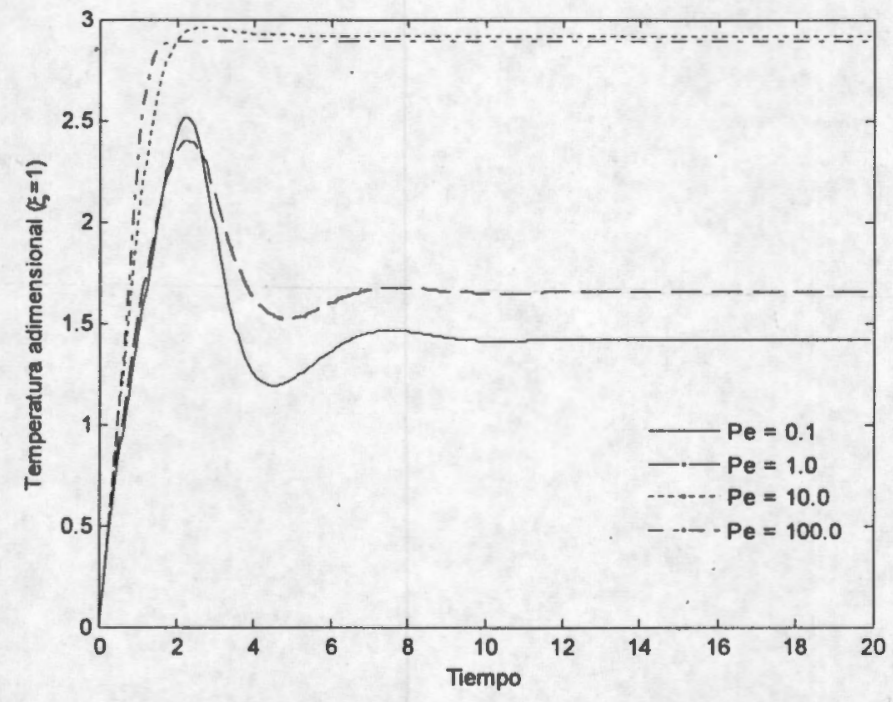

Figura 4-7 Perfil de temperatura cuasi lineal $(\xi=1)$ a diferentes números de Peclet en estado dinámico

Los casos mas difíciles de trabajar numéricamente son cuando el $P e=0.1$ y 1.0 dada la forma del perfil dinámico, como se ve en la Figura 4-7 que presenta el perfil de temperatura dinámico en $\xi=1$ y en las Figuras 4-8a-b se muestra el perfil dinámico en 3 dimensiones, la dinámica presentan un máximo de temperatura en 2.5 unidades adimensionales de tiempo en toda la dirección axial del reactor. Los casos donde el $P e=10.0$ y 100.0 no presentan ningún 
máximo de temperatura por lo que la dinámica es mas suave como se aprecia en las Figuras 4$8 \mathrm{c}-\mathrm{d}$.
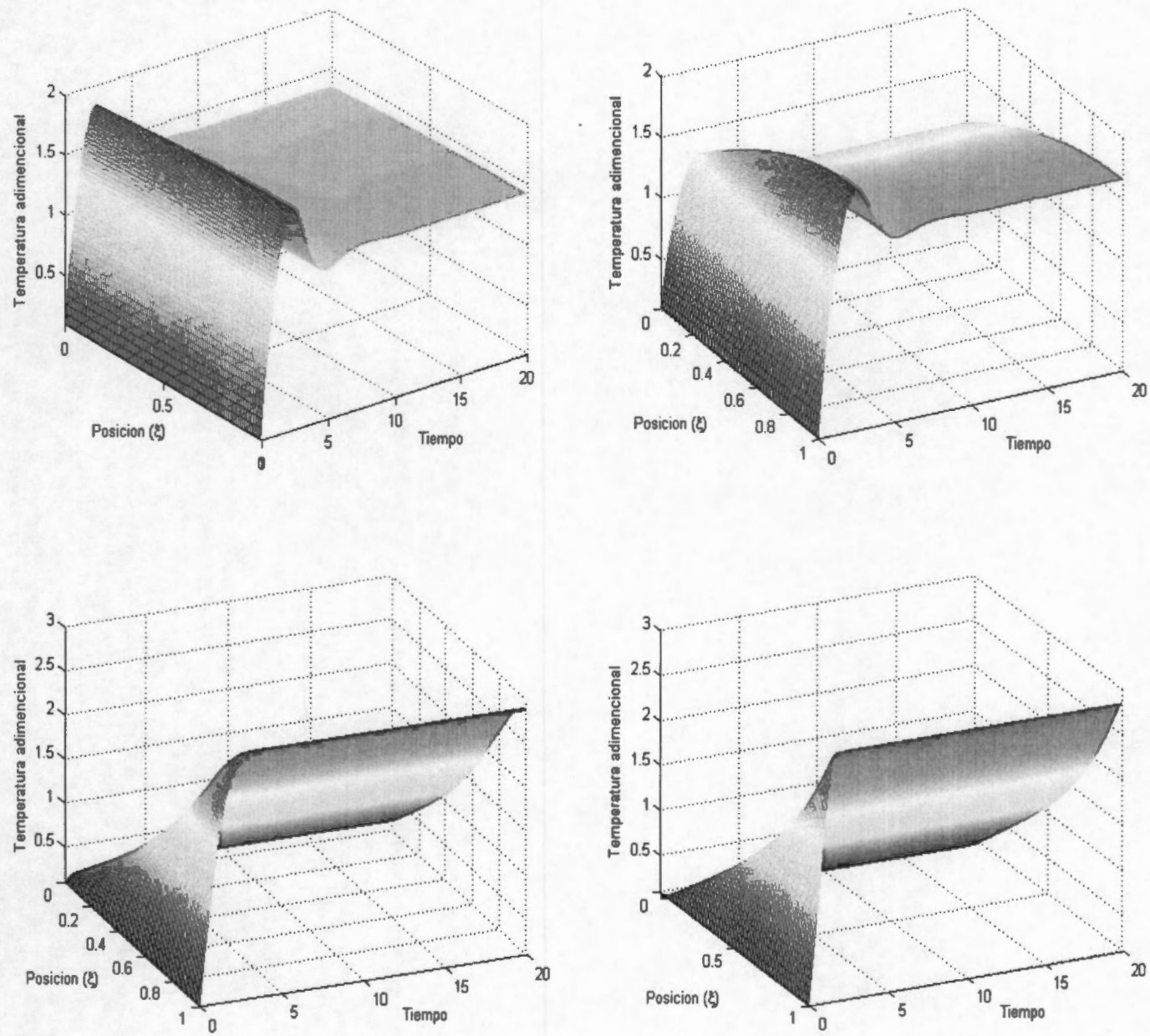

Figura 4-8 Solución dinámica del reactor tubular en 3 dimensiones a diferentes valores de Peclet, perfil cuasi lineal, a) $P e=0.1$, b) $P e=1.0$, c) $P e=10.0$ y d) $P e=100$.

En la Figura 4-9 se presentan los errores relativos (Ecuación 4-51) dinámicos, como era de esperarse los perfiles de temperatura $(\xi=1)$ con $P e=0.1$ y 1.0 muestran mayor error relativo que resto de los casos. Particularmente para el método de $\mathrm{CO}$ el uso de 10 nodos en la discretización es suficiente, debido a que la solución con más de 10 nodos no mejora la solución numérica significativamente, por tanto el método de CO siempre emplea menor número de nodos. Sin embargo los errores relativos calculados muestran que para los casos donde $P e=0.1$ y 1.0 el error es mayor para el método de $\mathrm{CO}$ que para DF y FEI (considerando que el numero de nodos en DF y FEI es mayor que $\mathrm{CO}$ ). Para el caso donde $\mathrm{Pe}$ $=10$ y 100 el método de CO tienen menor error relativo que DF y FEI, a pesar que DF y FEI emplean 200 y 500 nodos. Para nodos menores de 500, DF exhibe menor error relativo que FEI para los casos extremos $(P e=0.1$ y 100$)$, mientras que para los caso donde $P e=1$ y 10 las FEI mejoran a DF. 

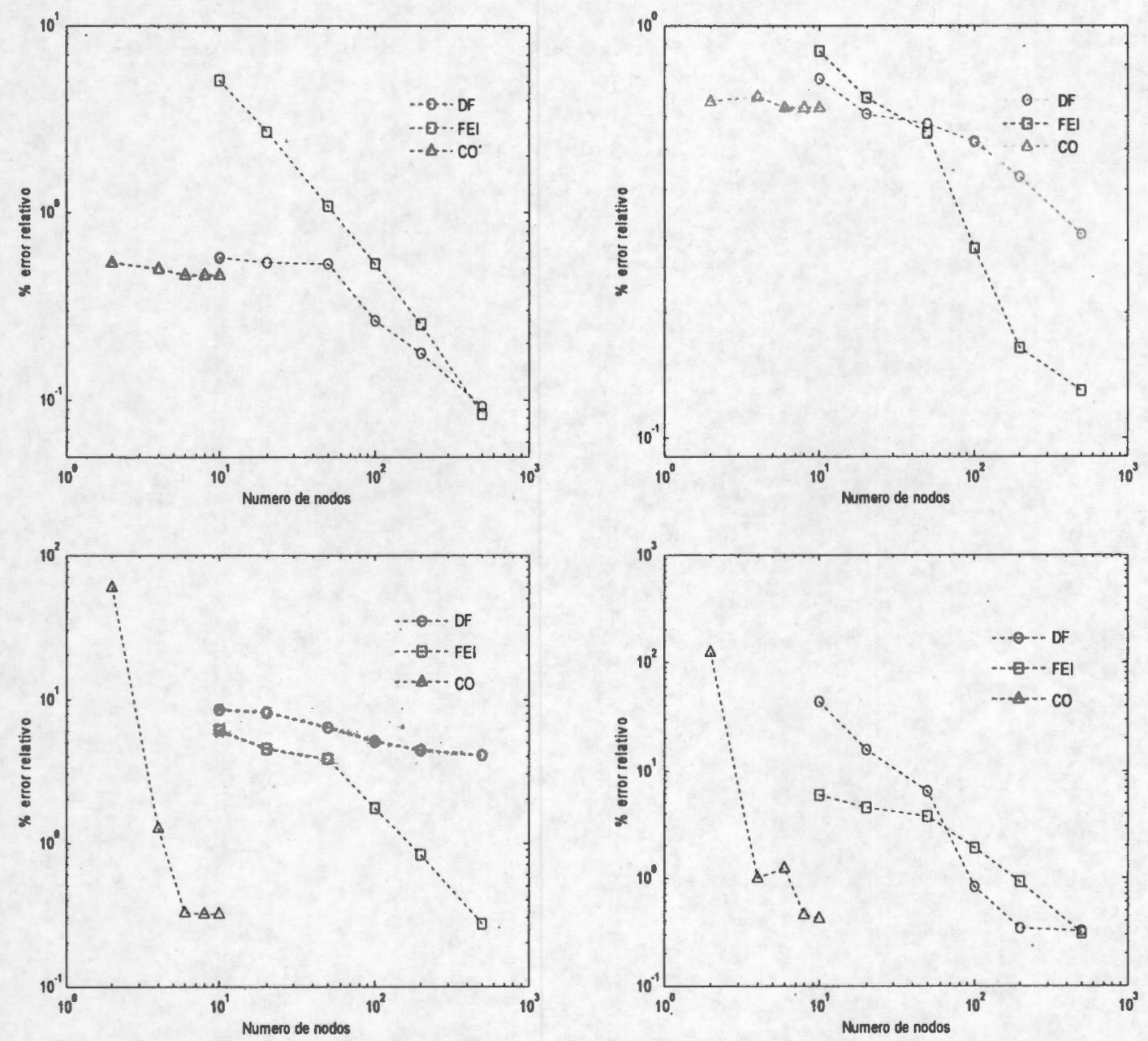

Figura 4-9 Aproximación numérica dinámica, caso: perfil cuasi lineal, error relativo vs. numero de nodos, a) $P e=0.1$, b) $P e=1.0$, c) $P e=10.0$ y d) $P e=100.0$

La Tabla 4-5 muestra los tiempos de cómputo correspondientes a la Figura 4-9. Así como en el caso estático los tiempos de cómputo para CO son menores que para DF y FEI. De manera general los tiempos de cómputo de DF son mayores a los requeridos por FEI.

Tabla 4-5 Tiempos de cómputo, perfil dinámico de temperatura cuasi lineal

\begin{tabular}{|c|c|c|c|c|}
\hline \multicolumn{5}{|c|}{ Colocación Ortogonal } \\
\hline Nodos & \multicolumn{4}{|c|}{ Tiempo (s) } \\
\hline & $\mathrm{Pe}=0.1$ & $\mathrm{Pe}=1.0$ & $\mathrm{Pe}=10.0$ & $\mathrm{Pe}=100.0$ \\
\hline 2 & 10.65 & 1.05 & 1.54 & 1.82 \\
\hline 4 & 104.34 & 1.91 & 1.64 & 1.93 \\
\hline 6 & 147.8 & 2.27 & 2.34 & 2.22 \\
\hline 8 & 148.22 & 4.72 & 2.51 & 2.11 \\
\hline 10 & 164.14 & 6.51 & 2.62 & 2.51 \\
\hline
\end{tabular}




\begin{tabular}{|c|c|c|c|c|}
\hline \multicolumn{5}{|c|}{ Diferencias Finitas } \\
\hline Nodos & \multicolumn{5}{|c|}{ Tiempo $(\mathrm{s})$} \\
\hline & $\mathrm{Pe}=0.1$ & $\mathrm{Pe}=1.0$ & $\mathrm{Pe}=10.0$ & $\mathrm{Pe}=100.0$ \\
\hline 10 & 9.10 & 3.08 & 1.46 & 1.84 \\
\hline 20 & 40.81 & 4.97 & 1.87 & 1.37 \\
\hline 50 & 201.2 & 33.51 & 2.84 & 1.71 \\
\hline 100 & 1808.47 & 184.04 & 11.31 & 4.21 \\
\hline 200 & 9671.5 & 1140 & 60.57 & 16.17 \\
\hline 500 & 130432 & 2904 & 744.2 & 208.94 \\
\hline
\end{tabular}

\begin{tabular}{|c|c|c|c|c|}
\hline \multicolumn{5}{|c|}{ Formulaciones de Ecuaciones Integrales } \\
\hline Nodos & \multicolumn{5}{|c|}{ Tiempo (s) } \\
\hline & $\mathrm{Pe}=0.1$ & $\mathrm{Pe}=1.0$ & $\mathrm{Pe}=10.0$ & $\mathrm{Pe}=100.0$ \\
\hline 10 & 16.75 & 9.24 & 2.41 & 1.61 \\
\hline 20 & 25.65 & 10.74 & 3.01 & 2.3 \\
\hline 50 & 43.56 & 14.54 & 4.31 & 3.21 \\
\hline 100 & 168.5 & 54.34 & 14.87 & 8.67 \\
\hline 200 & 587.9 & 183.1 & 50.99 & 29.1 \\
\hline 500 & 8245.78 & 1095.76 & 304.06 & 169.52 \\
\hline
\end{tabular}

\subsubsection{Perfil de temperatura con hot spot}

Para el análisis del sistema en estado dinámico cuando el perfil de temperatura presenta un hot spot consideramos los mismos criterios que el caso anterior. Calculamos los perfiles dinámicos de la temperatura del reactor en $\xi=1$ (Figura 4-10) y los perfiles dinámicos en 3 dimensiones (Figura 4-11) para diferentes valores de Peclet $\left(P e=\left[\begin{array}{llll}0.1 & 1.0 & 10.0 & 100.0\end{array}\right]\right)$. La Figura 4-10 muestra que el perfil de temperatura $(\xi=1)$ tiene máximo en el tiempo adimensional $\tau=0.5$ para diferentes números de Peclet, con la variante que en cada caso la temperatura converge de manera distinta.

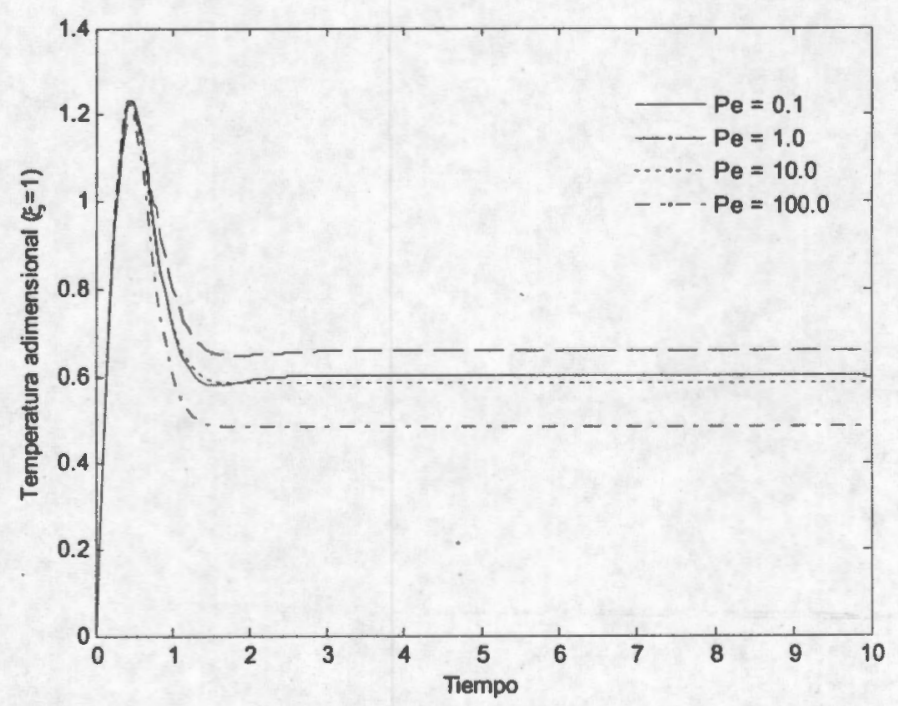

Figura 4-10 Perfil de temperatura cuasi lineal $(\xi=1)$ a diferentes números de Peclet en estado dinámico 
En la Figura 4-11 se ve mas claro el comportamiento dinámico de la temperatura a lo largo del reactor y como evoluciona en el tiempo a diferentes números de Peclet. El hot spot disminuye a medida que el Peclet tiende a cero y se hace mas pronunciado cuando el Peclet es grande.
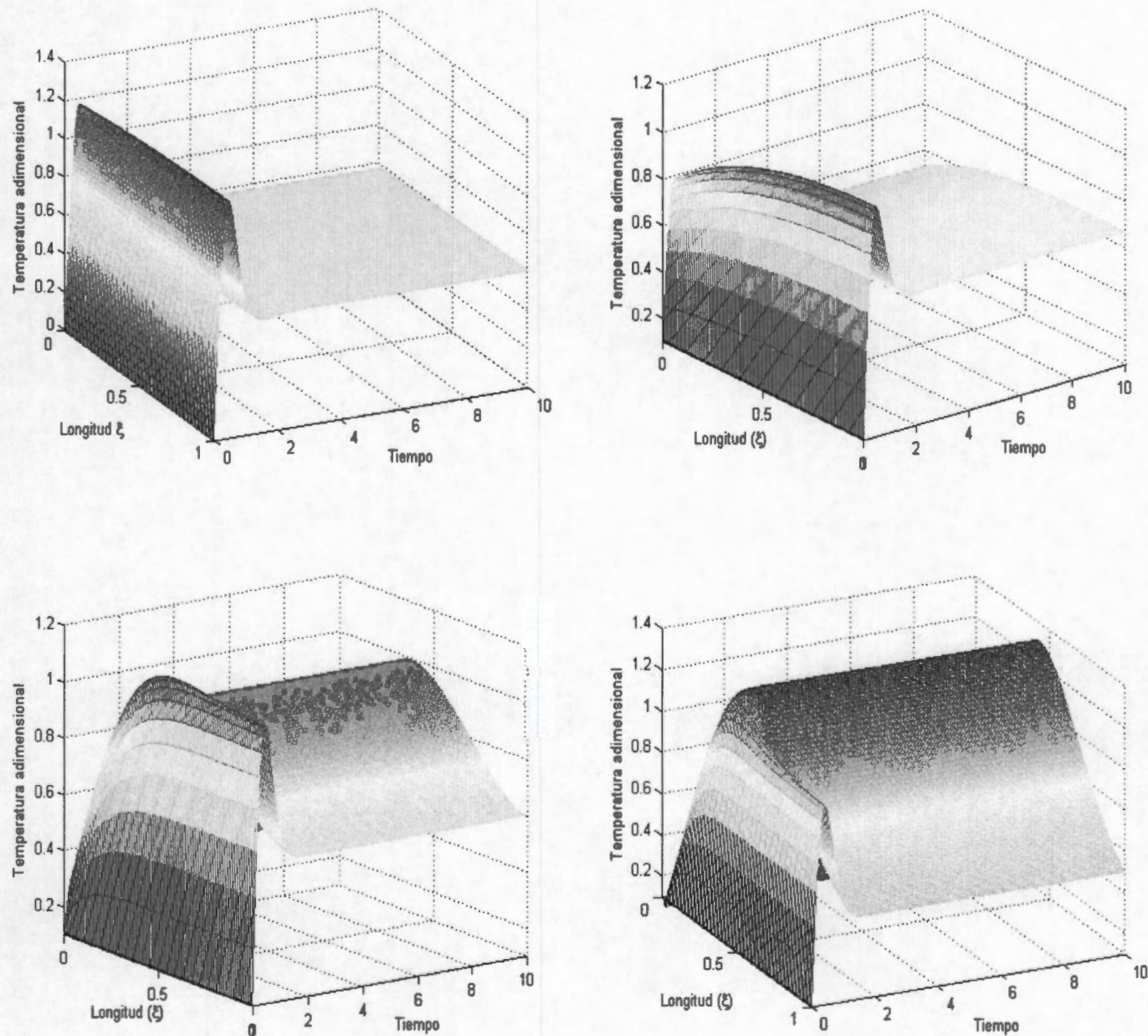

Figura 4-11 Solución dinámica del reactor tubular en 3 dimensiones a diferentes valores de Peclet, perfil con hot spot, a) $P e=0.1$, b) $P e=1.0$, c) $P e=10.0$ y d) $P e=100$

La Figura 4-12 muestra los errores relativos. Debido a la complicada estructura de la solución dinámica, el método de $\mathrm{CO}$ muestra un mayor error que los métodos de DF y FEI para los cuatro casos. Para el caso donde el $P e=0.1$ el método de DF se ve mas efectivo que las FEI a un numero de nodos menor de 500, pero para el resto de los casos el método de FEI tienen mejor aproximación numérica.

Los tiempos de cómputo estimados muestran que nuevamente el método de $\mathrm{CO}$ requiere de menor tiempo de cómputo frente a los demás esquemas y las FEI emplean menos tiempo de cómputo que DF. 

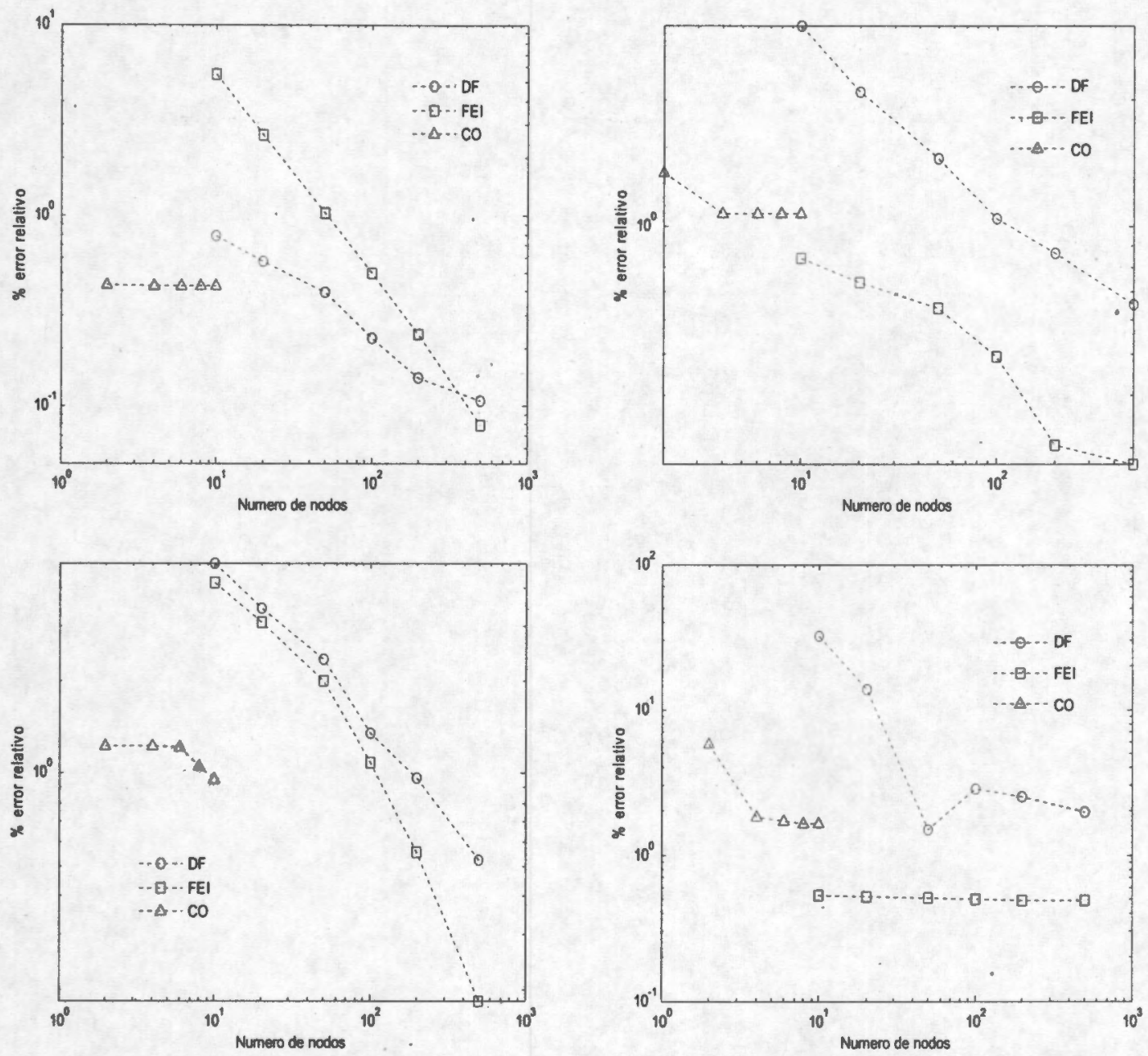

Figura 4-12 Aproximación numérica dinámica, caso: perfil con hot spot, error relativo vs. numero de nodos, a) $P e=0.1$, b) $P e=1.0$, c) $P e=10.0$ y d) $P e=100.0$

En esta parte hemos comparado los tres métodos de discretización para el modelo del reactor tubular en estado estacionario y en estado dinámico, calculando el error relativo basado en la solución del sistema por DF empleando 2000 nodos, cuya solución se ha considerado "exacta". Para verificar la capacidad de aproximación numérica se ha resulto el sistema variando los números de Peclet en el rango de $10^{-1}-10^{2}$. Los resultados muestran que el método de $\mathrm{CO}$ requiere de un número menor de nodos que DF y FEI, sin embargo existen regiones del numero de Peclet donde CO tiene mayor error relativo que DF y FEI (en estado estacionario $P e=100$; estado dinámico: $i$ ) cuasi lineal $P e=0.1$ y 1.0 y ii) hot spot todo el rango de valores de $P e$ ). Por otro lado existen regiones donde DF tiene mejor desempeño que FEI, sin embargo FEI requiere de tiempos de cómputo menores que DF.

Las FEI muestran menor error relativo a medida que el número de nodos empleados para discretizar el sistema es mayor, este comportamiento es constante para soluciones dinámicas o estáticas. El análisis estático muestra que FEI requiere de un menor mallado que DF para el mismo error relativo, pero en algunos casos dinámicos los errores calculados para FEI son mayores que para DF empleando el mismo numero de nodos, pero en general cuando se emplean 500 nodos los valores del error relativo son semejantes o mejores que los de DF. El 
tiempo de computo requerido para la solución del sistema mediante FEI siempre es menor que el usado en DF.

Los resultados reportados en la literatura acerca de los diferentes métodos presenta un comparativo de las FEIs frente esquemas de DF, concluyendo que las FEIs requieren de menor mallado y menor tiempo de computo (Kesten, 1969; Valdez-Parada, 2007); por otro lado la comparación de DF con CO muestra que en cierto rango de operación del reactor el método de CO tiene mejor desempeño (Finlayson, 1980) sin embargo existen problemas donde la solución presenta cambios bruscos y el método de $\mathrm{CO}$ presenta grandes errores de aproximación o no es inaplicable el método.

Tabla 4-6 Tiempos de cómputo, perfil dinámico de temperatura con hot spot

\begin{tabular}{|c|c|c|c|c|}
\hline \multicolumn{5}{|c|}{ Colocación Ortogonal } \\
\hline Nodos & \multicolumn{4}{|c|}{ Tiempo (s) } \\
\hline & $\mathrm{Pe}=0.1$ & $\mathrm{Pe}=1.0$ & $\mathrm{Pe}=10.0$ & $\mathrm{Pe}=100.0$ \\
\hline 2 & 5.32 & 0.78 & 0.87 & 0.79 \\
\hline 4 & 6.80 & 0.79 & 0.93 & 1.1 \\
\hline 6 & 9.07 & 1.10 & 0.94 & 1.16 \\
\hline 8 & 10.16 & 2.20 & 1.20 & 1.24 \\
\hline 10 & 12.57 & 2.47 & 1.34 & 2.47 \\
\hline
\end{tabular}

\begin{tabular}{|c|c|c|c|c|}
\hline \multicolumn{5}{|c|}{ Diferencias Finitas } \\
\hline Nodos & \multicolumn{5}{|c|}{ Tiempo (s) } \\
\hline & $\mathrm{Pe}=0.1$ & $\mathrm{Pe}=1.0$ & $\mathrm{Pe}=10.0$ & $\mathrm{Pe}=100.0$ \\
\hline 10 & 4.41 & 2.34 & 1.06 & 1.66 \\
\hline 20 & 16.21 & 4.04 & 1.56 & 1.35 \\
\hline 50 & 125.5 & 20.62 & 3.24 & 2.01 \\
\hline 100 & 628.68 & 104.78 & 11.38 & 2.94 \\
\hline 200 & 4824.4 & 603.05 & 62.07 & 9.12 \\
\hline 500 & 73895.75 & 7778.5 & 774.1 & 84.3 \\
\hline
\end{tabular}

\begin{tabular}{|c|c|c|c|c|}
\hline \multicolumn{5}{|c|}{ Formulaciones de Ecuaciones Integrales } \\
\hline Nodos & \multicolumn{5}{|c|}{ Tiempo (s) } \\
\hline & $\mathrm{Pe}=0.1$ & $\mathrm{Pe}=1.0$ & $\mathrm{Pe}=10.0$ & $\mathrm{Pe}=100.0$ \\
\hline 10 & 17.65 & 8.79 & 2.91 & 1.69 \\
\hline 20 & 32.45 & 10.28 & 2.70 & 1.85 \\
\hline 50 & 76.54 & 23.65 & 2.87 & 2.02 \\
\hline 100 & 135.4 & 36.46 & 8.04 & 5.31 \\
\hline 200 & 867.54 & 178.32 & 27.27 & 17.93 \\
\hline 500 & 3978.5 & 466.68 & 153.23 & 97.47 \\
\hline
\end{tabular}




\section{Capítulo 5}

\section{Simulaciones a}

\section{Lazo Cerrado}

En este capítulo se presenta un análisis dinámico del sistema verificando la aplicación de técnicas de control en cascada, se presentan las simulaciones numéricas a lazo cerrado del reactor tubular empleando técnicas de control en cascada convencional y control en cascada empleando una temperatura promedio ponderada en el lazo de temperatura, la temperatura promedio se determina a partir de la medición de temperatura en tres puntos a lo largo de la dirección axial del reactor.

\subsection{Introducción}

El control en cascada es una de las herramientas más útiles para el diseño de controles avanzados. Su utilidad radica en su capacidad para rechazar perturbaciones y mejorar el desempeño de los controladores. Este tipo de estrategia se usa en los procesos químicos debido a que existen variables con medidas retardadas (reactores químicos) o procesos de gran capacitancia (columnas de destilación). En los cuales la implementación de una técnica de control convencional, como el uso de controladores con estructura proporcional e integral implica retardos en las acciones de control y mal desempeño de los controladores ya que las perturbaciones y los ruidos de medición no serán detectados al tiempo que ocurren y por lo tanto el control tardará en corregir la acción de control. 
La idea de usar control en cascada se originó con el problema de rechazo de perturbaciones cuyos efectos se detectan más rápidamente en una salida del control secundario que en la variable controlada. Entonces, el uso de control en cascada es recomendado para aquellos procesos en los cuales la dinámica de un lazo de control secundario es más rápida que la dinámica del proceso primario (Stephanopoulos, 1984). En estas condiciones la interacción entre los dos esquemas de control formados es prácticamente nula, debido a la diferencia en sus dinámicas y por la cual se dice que no se presentan problemas de sintonizado en el esquema de control en cascada.
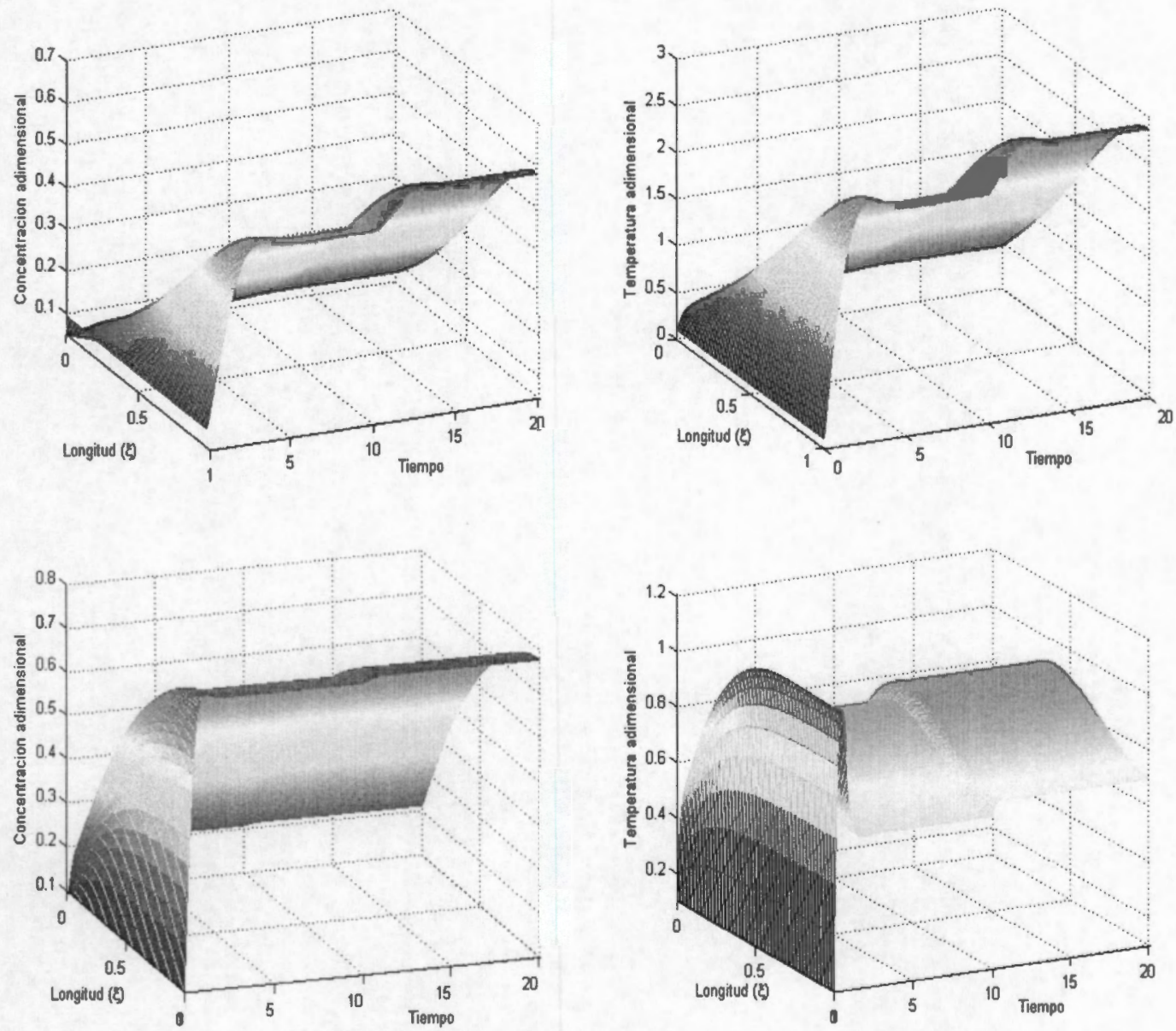

Figura 5-1 Respuestas a perturbación en +10 en $\varpi$, a) perfil de concentración b) perfil de temperatura sin hot spot, $\mathrm{c}$ ) perfil de concentración y d) perfil de temperatura con hot spot

\subsection{Análisis dinámico}

La variable propuesta a ser la variable de control es la temperatura adimensional de la camisa de enfriamiento ( $\varpi)$, por lo que es necesario ver el efecto de cambios en escalón de esta variable a lo largo del reactor, caracterizando así la conveniencia de la implementación de una estrategia de control en cascada. 
En la Figura 5-1 se muestra la respuesta a una perturbación en escalón de $+10 \%$ en $\varpi$ a $\tau$ $=10$, en el perfil de temperatura tanto en la dinámica lineal como en la que presenta hot spot a lo largo del reactor, en la Figura 5-1 a-b observamos la respuesta en la dinámica del sistema que tiene perfil lineal de temperatura y en la Figura 5-1c-d la respuesta del sistema con dinámica con hot spot. Se aprecia en la Figura 5-1 que una perturbación en escalón del $+10 \%$ en la temperatura de enfriamiento tiene un efecto mas pronunciado en la dinámica sin hot spot que con hot spot, sin embargo en ambas dinámicas se cuenta con una favorable respuesta como para emplear a $\varpi$ como la variable a manipular.

Para el diseño del control en cascada se debe satisfacer que la dinámica del lazo secundario debe tener una respuesta más rápida que el lazo primario, por tanto cuantificaremos la respuesta de concentración y la temperatura en diferentes posiciones a lo largo del reactor frente a una perturbación en $\varpi$. En la Figura 5-2 se muestra las respuestas sin hot spot de la concentración en $\xi(1)$ (variable a controlar) y la temperatura del reactor en $\xi(0.2), \xi(0.5)$ y $\xi(0.8)$ que son las posibles posiciones para el sensor de temperatura en el diseño de control en cascada, mientras la respuestas con hot spot se muestran en la Figura 5-3.
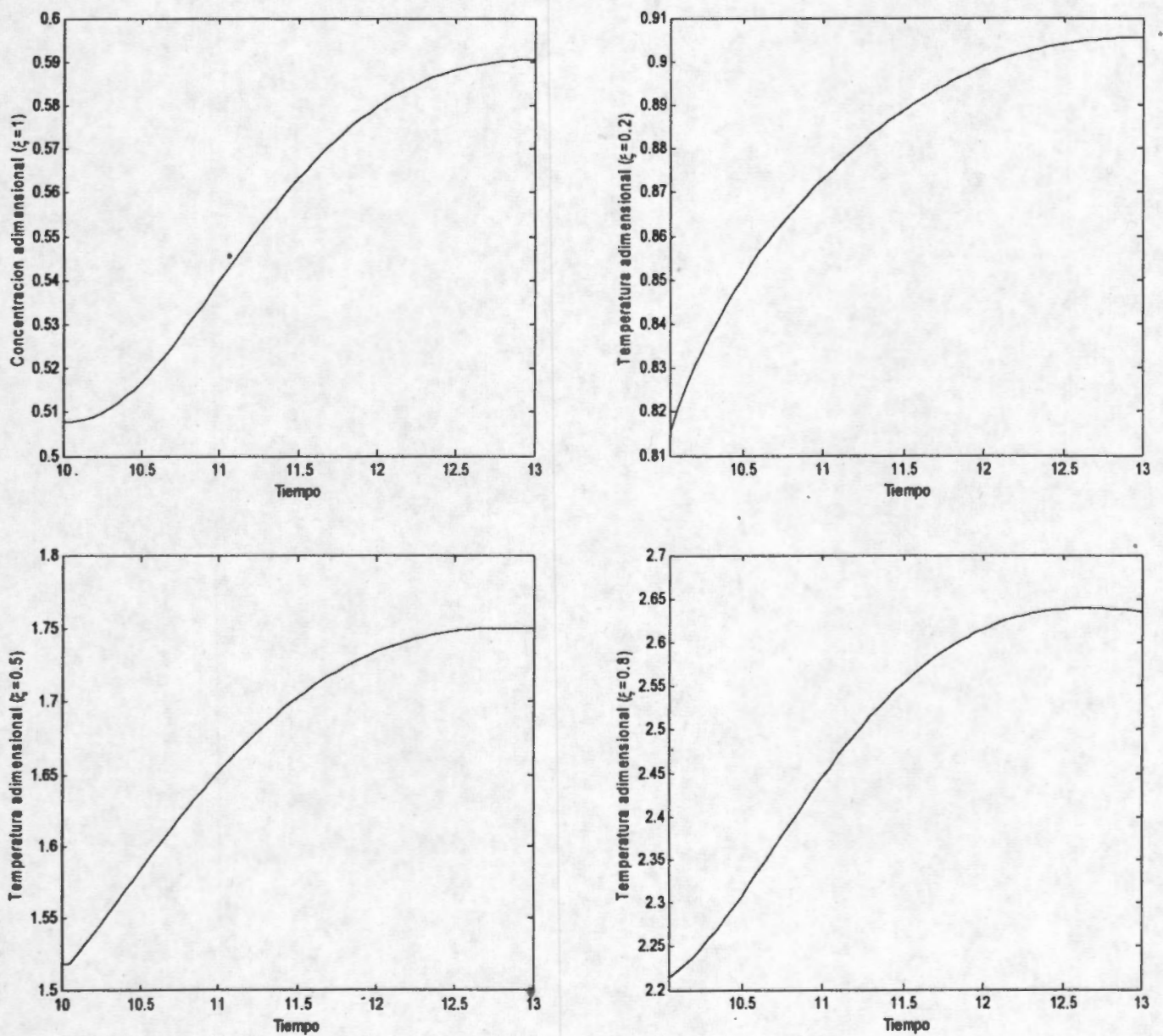

Figura 5-2 Respuestas a perturbación en +10 en $\varpi \mathrm{a} t=10$, dinámica sin hot Sp̉ot a) perfil de concentración en $\xi(1)$ b) perfil de temperatura $\xi(0.2)$, c) $\xi(0.5)$ y d) $\xi(0.8)$ 
En la Figura 5-2 se muestra la respuesta a perturbaciones de la temperatura en sus diferentes posiciones $(0.2,0.5$ y 0.8$)$ a lo largo del reactor, la respuesta es mas rápida a medida que nos acercamos a la entrada de los reactantes al reactor, es decir, que la velocidad de respuesta se comportan como: $\xi(0.2)>\xi(0.5)>\xi(0.8)$, así mismo los perfiles de temperatura tienen una respuesta mas rápida que el perfil de concentraciones por lo que es posible proponer un esquema de control en cascada.

Los perfiles de temperatura cuando existe un punto caliente suelen tener trayectorias variadas en diferentes puntos alrededor del hot spot, sin embargo las velocidades de respuestas a perturbaciones van de mayor a menor de la entrada a la salida del reactor, como se ve en la Figura 5-3. La velocidad de respuesta del perfil de concentraciones es menor que el del perfil de temperaturas por lo que es razonable proponer un esquema de control en cascada.
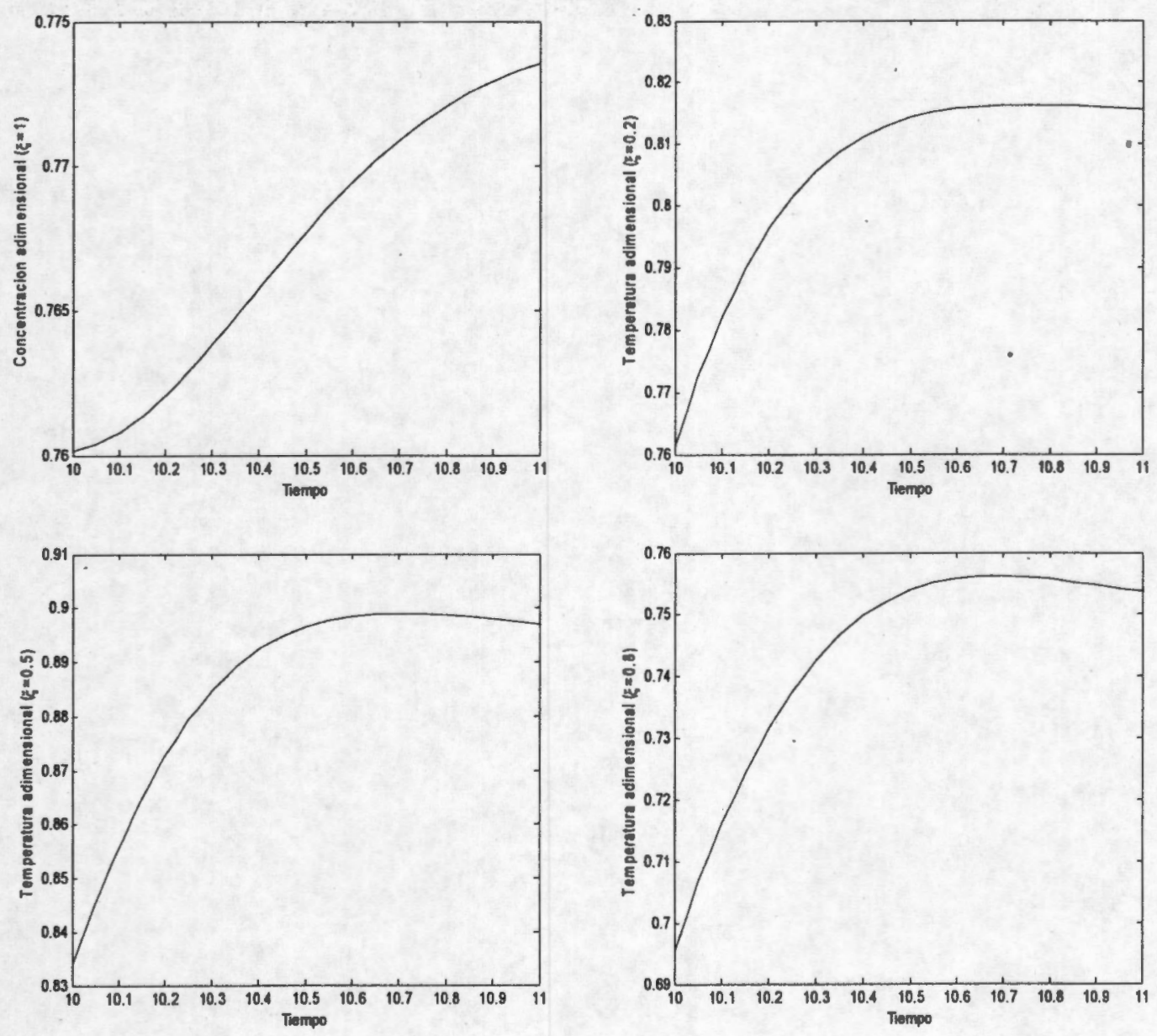

Figura 5-3 Respuestas a perturbación en +10 en $\varpi \mathrm{a} t=10$, dinámica con hot spot a) perfil de concentración en $\xi(1)$ b) perfil de temperatura $\xi(0.2)$, c) $\xi(0.5)$ y d) $\xi(0.8)$ 


\subsection{Dinámica a lazo cerrado}

El objetivo de control en el reactor tubular con dispersión axial es mantener la fracción de conversión a la salida del reactor fija, es decir, sin variaciones frente a perturbaciones (cambios en composición y temperatura a la entrada del reactor). Con objeto de comparar se implemento al sistema 3 tipos de controladores, Pl clásico controlando conversión a la salida del reactor mediante la manipulación de la temperatura de enfriamiento $(\varpi)$, un controlador en cascada convencional de conversión-temperatura y una modificación al esquema clásico de control en cascada de conversión-temperatura promedio ponderada, donde la temperatura promedio viene de la medición de tres puntos a lo largo del reactor como se detallo en el capítulo 3.

\subsubsection{Control en cascada convencional}

El diseño del control en cascada de concentración-temperatura desea controlar la conversión a la salida del reactor mediante la regulación de la temperatura en un punto del reactor (control maestro). Los sensores de temperatura propuestos para el lazo de temperatura son distribuidos a lo largo del reactor en $\xi(0.2), \xi(0.5)$ y $\xi(0.8)$ implementando un controlador en cada punto espacial con el objeto de determinar la mejor posición del sensor para el control en cascada. El lazo de temperatura es regulada mediante la manipulación de la temperatura adimensional de la camisa de enfriamiento $\varpi$, (control Esclavo).

\subsubsection{Perfil de temperatura cuasi-lineal}

El diseño de control esta constituido por modelos (MPOTM), estos modelos son calculados a partir de la respuesta dinámica de una perturbación en escalón inducido por un cambio en la temperatura de camisa $(\varpi)$ del $10 \%$ (Ecuaciones 3-1 y 3-2), los parámetros de los controladores son calculados mediante las reglas de sintonizado reportados en (Morari y Zafiriou, 1989).

El esquema de control en cascada convencional de composición-temperatura se basa en las Ecuaciones (3-2) y (3-5) desarrolladas en el capitulo 3, empleando únicamente una retroalimentación Integral (I) para el lazo de composición y una retroalimentación Proporcional-Integral (PI) para el lazo de temperatura. El lazo de temperatura es propuesto para tres posiciones del sensor de temperatura en $\xi=\{0.2,0.5,0.8)$. Los parámetros del controlador son determinados acorde a las reglas de sintonizado (IMC) (ver sección 3-4), la ganancia integral del lazo de composición es $K_{I, y}=\{1.0452,2.6567,4.5381\}$ y los valores de las constantes del controlador PI para el lazo de temperatura son: $K_{c, w}=\{0.8081,0.3179$, $0.1861\}$ y $\tau_{l, w}=\{0.7182,1.1367,1.2704\}$ para las posiciones $\xi=\{0.2,0.5,0.8)$ respectivamente.

. Una vez que el reactor es estabilizado a una concentración adimensional determinada $(y=$ 0.5 ), para los tres casos el reactor fue sujeto a una secuencia de perturbaciones en la alimentación: i) a $\tau=20$ un $+10 \%$ en la concentración de entrada al reactor; a $\tau=60$ un $+20 \%$ en la temperatura de entrada al reactor y finalmente en $t=100$ un $-10 \%$ en la temperatura de entrada al reactor. El desempeño del controlador en cascada frente a las perturbaciones arriba mencionadas, empleando diferentes mediciones de temperatura a lo largo del reactor se muestra en la Figura 5-4a. 

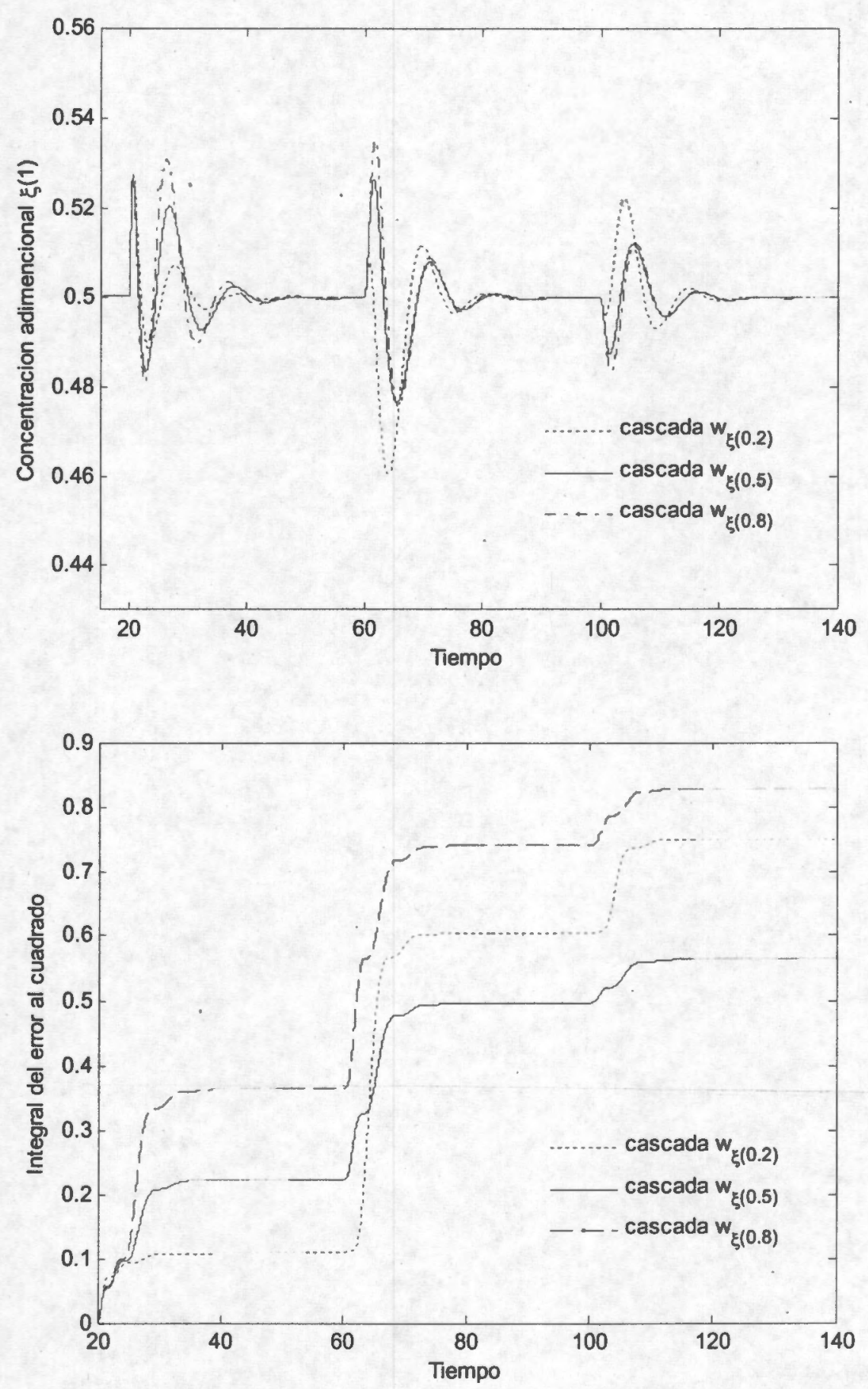

Figura 5-4 Control en cascada convencional con sensor de temperatura en posición adimensional 0.20 .5 y 0.8: perfil cuasi-lineal, a) regulación de concentración a $y_{\text {ref. }}=0.5$ frente a perturbaciones $\mathrm{y} b$ ) integral del error al cuadrado.

En la Figura 5-4b se presenta la integral del error de los tres casos donde se aprecia que de manera general que el controlador en cascada con sensor de temperatura en la posición $\xi(0.5)$ tiene un mejor desempeño. Sin embargo, se observa en la Figura 5-4b que la respuesta del controlador a diferentes perturbaciones depende de la posición del sensor de temperatura, a perturbaciones en la concentración el control con lazo de temperatura en $\xi(0.2)$ tiene una 
respuesta mas rápida que $\xi(0.8)$, por otro lado, frente a perturbaciones en la temperatura el control con lazo de temperatura en $\xi(0.8)$ la respuesta en mas rápida que en $\xi(0.2)$, esto nos lleva reflexionar que lo ideal seria poder explotar las propiedades de los controladores combinados, lo que nos conduce al esquema de control en cascada empleando un promedio de las tres mediciones.
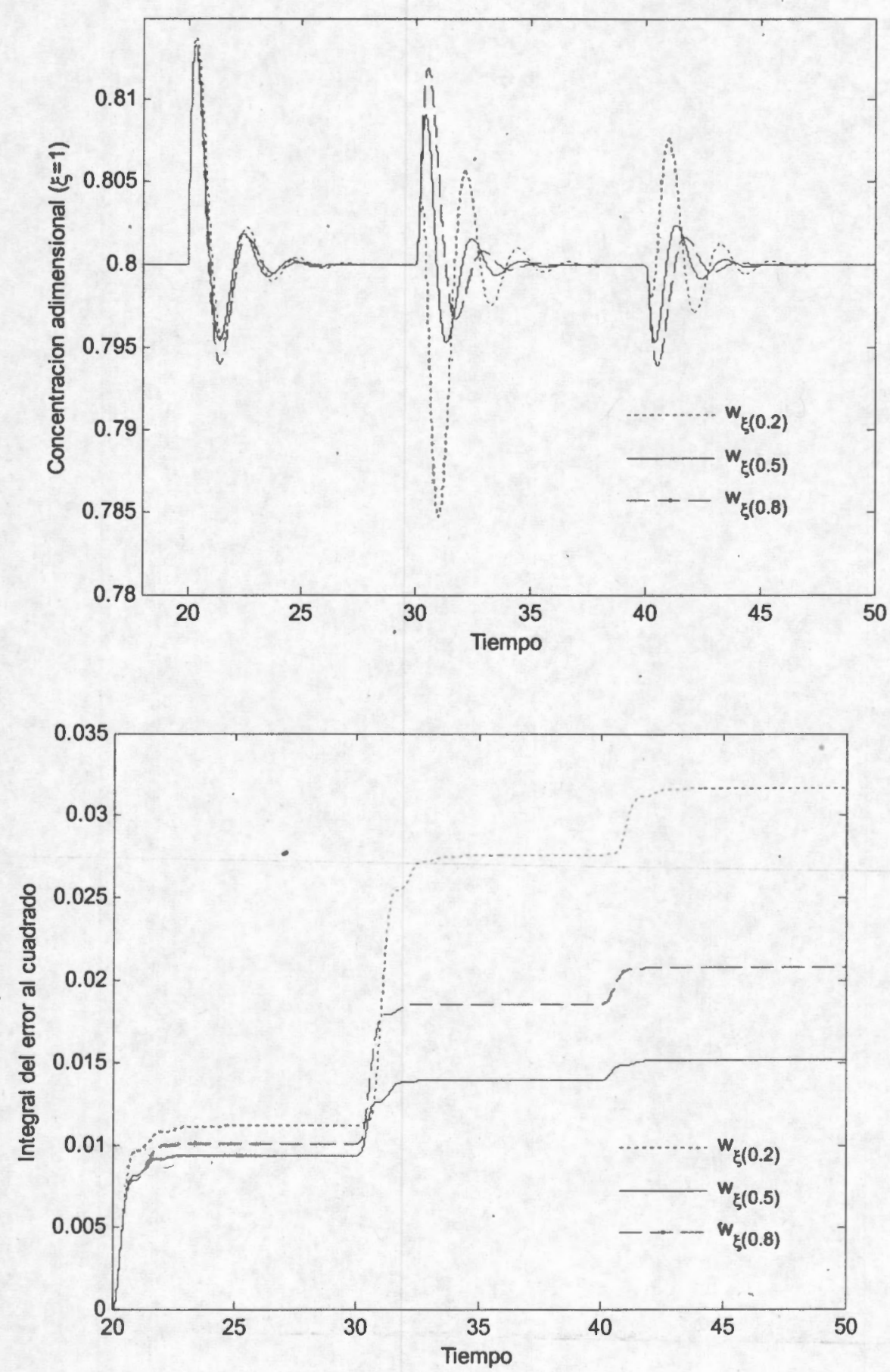

Figura 5-5 Control en cascada convencional con sensor de temperatura en posición adimensional 0.20 .5 y 0.8 : perfil con hot spot, a) regulación de concentración a $y_{\text {ref }}=0.8$ frente a perturbaciones y b) integral del error al cuadrado. 


\subsubsection{Perfil de temperatura con hot spot}

Para este caso el control en cascada con una sola medición se plantea como en la sección anterior en tres posiciones distintas $\xi=\{0.2,0.5,0.8\}$, considerando que $\xi=0.2-0.5$ corresponde a la localización típica de un hot spot en los reactores tubulares. Para las simulaciones numéricas empleamos los siguientes valores de los parámetros del controlador: la ganancia integral del lazo de composición es $K_{I, y}=\{15.7894,18.2753,16.3443\}$ y las constantes del controlador PI para el lazo de temperatura son: $K_{c, w}=\{1.2939,1.1093,1.2403\}$ y $\tau_{l, w}=\{0.1562,0.1644,0.1565\}$ para las posiciones $\xi=\{0.2,0.5,0.8)$ respectivamente.

El reactor fue estabilizado a una composición de $y=0.8$ y posteriormente el reactor fue sujeto a una secuencia de perturbaciones en las condiciones de alimentación: i) a $\tau=20$ un $+10 \%$ en la concentración de entrada al reactor; a $\tau=30$ un $+20 \%$ en la temperatura de entrada al reactory finalmente en $\tau=40$ un $-10 \%$ en la temperatura de entrada al reactor. La Figura 5-5a muestra el desempeño de los controladores. Como en el caso anterior (perfil de temperatura cuasi-lineal) el controlador con sensor de temperatura en $\xi=(0.5)$ tiene el mejor desempeño (ver Figura 5-5b).

Es interesante señalar que el comportamiento del contralor con sensor de temperatura en $\xi$ $=(0.2)$ es mejor que en $\xi=(0.8)$ para el caso con perfil cuasi-lineal (ver Figura 5-4b) y que para el caso con perfil con hot spot las repuestas son contrarias (ver Figura 5-5b), esta situación puede ser explicado de la siguiente forma: cuando el perfil presenta hot spot la dinámica cercana a la entrada del reactor es dominada por el calor de reacción dejando en segundo plano el transporte en la pared, así que es mas difícil controlar la temperatura del reactor mediante la temperatura de enfriamiento y en consecuencia un pobre desempeño del controlador, por otro lado cuando tenemos una dinámica con perfil de temperatura cuasilineal, un sensor de temperatura localizado cercano a la entrada del reactor se ve favorecido por la pronta detección en los cambios en la alimentación del reactor y toma rápidas acciones de corrección.

\subsubsection{Control en cascada con temperatura promedio ponderada}

Para este esquema de control se hace una modificación al control en cascada convencional remplazando el lazo secundario guiado por la medición de la temperatura en un punto del reactor por el promedio de tres mediciones de temperatura $\left(w_{a v}\right)$ virtual, llamada virtual debido a que esta temperatura no existe físicamente es solo un promedio ponderado de tres mediciones en diferentes puntos del reactor (Ecuación 3-6). Los puntos donde se colocan los sensores de temperatura en el reactor se escogieron de tal forma en que abarquen simétricamente la totalidad del reactor.

\subsubsection{Perfil de temperatura cuasi-lineal}

Para el diseño del controlador en cascada concentración - temperatura promedio ponderada se emplean las Ecuaciones (3-5) y (3-7). Los valores de los parámetros del controlador son determinados para cada tercia de valores de ponderación. El peso de los valores $\alpha, \beta$ y $\gamma$ es asignado a partir de las respuestas del controlador frente a las perturbaciones en la 
alimentación siguientes: $i$ ) a $\tau=20$ un $+10 \%$ en la concentración de entrada al reactor; a $\tau=$ 60 un $+20 \%$ en la temperatura de entrada al reactor y finalmente en $\tau=100$ un $-10 \%$ en la temperatura de entrada al reactor. La integral del error al cuadrado correspondiente a las combinaciones de parámetros $\alpha, \beta$ y $\gamma$ más representativos se muestran en la Figura 5-6, donde se aprecia que el controlador con mayor peso al sensor en $\xi(0.2)$ tiene un mejor desempeño, así mismo la distribución de peso entre los sensores en $\xi(0.2)$ y $\xi(0.5)$ dan un buen desempeño.

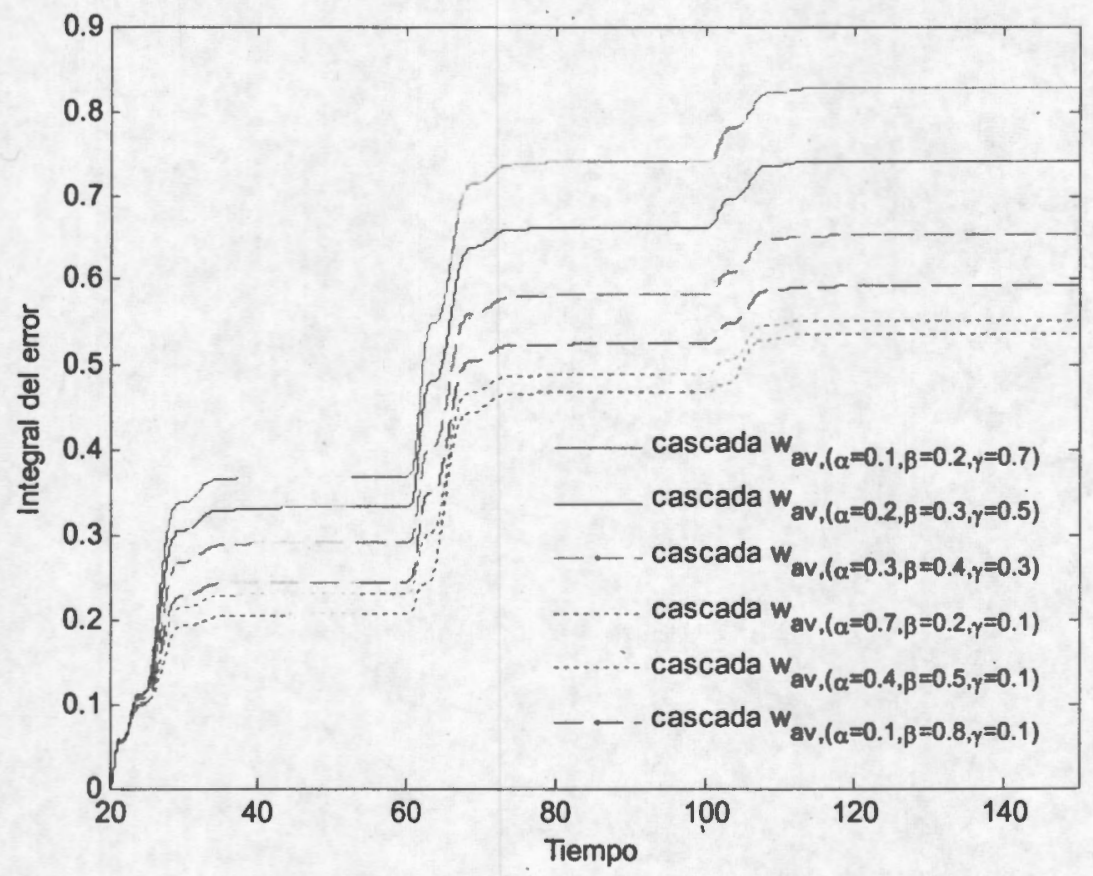

Figura 5-6 Integral del error al cuadrado de la respuesta a perturbaciones del controlador en cascada con $w_{a v}$ para determinar los valores de ponderación $\alpha, \beta$ y $\gamma$; perfil cuasi-lineal.

\subsubsection{Perfil de temperatura con hot spot}

La dinámica con hot spot tiene una respuesta más rápida por lo que reducimos el tiempo de la simulación a $\tau=50$ unidades adimensionales, introduciendo la siguiente secuencia de perturbaciones: $i$ ) a $\tau=20$ un $+10 \%$ en la concentración de entrada al reactor; a $\tau=30$ un $+20 \%$ en la temperatura de entrada al reactor y finalmente en $\tau=40$ un $-10 \%$ en la temperatura de entrada al reactor $\mathrm{La}$ determinación de los parámetros de ponderación $(\alpha, \beta$ y $\gamma$ ) se realiza de manera Heurística proponiendo variaciones en la combinación de los valores de ponderación. La Figura 5-7 muestra las combinaciones de valores más representativos, determinando que el controlador tiene el mejor desempeño cuando el peso esta cargado al sensor en $\xi=(0.5)$. 


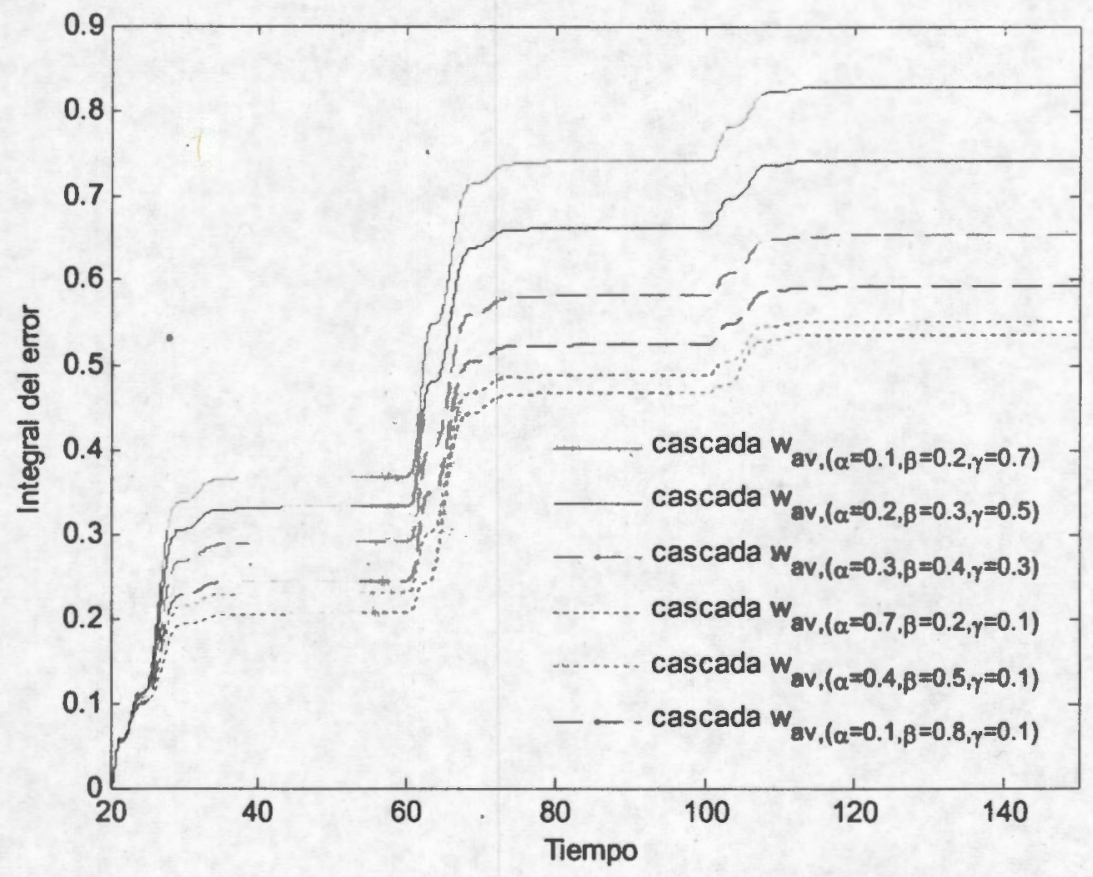

Figura 5-7 Integral del error al cuadrado de la respuesta a perturbaciones del controlador en cascada con $w_{a v}$ para determinar los valores de ponderación $\alpha, \beta$ y $\gamma$; perfil con hot spot

\subsubsection{Comparación de los esquemas de control}

Para fines de comparación diseñamos un controlador con un solo lazo de retroalimentación, regulando la composición mediante la manipulación de la camisa de enfriamiento. El controlador esta basado en (3-1); en modelos (E/S) de primer orden más tiempo muerto es suficiente emplear una retroalimentación PI, los parámetros del controlador son: $K_{c, a y}=0.7116$ y $\tau_{I, \text { ay }}=1.4987$ calculados en base a las reglas IMC (Morari y Zifiriou, 1989). Consideramos los valores de ponderación en $\alpha=0.7, \beta=0.2$ y $\gamma=0.1$ para hacer la comparación de control propuesto con el controlador en cascada convencional con sensor de temperatura localizado en $\xi(0.5)$ y el controlador con un solo lazo de retroalimentación.

En la Figura 5-8a se muestra el desempeño de los diferentes controladores frente a las perturbaciones arriba mencionadas. El cuadrado del error se muestra en la Figura 5-8b, observamos que el comportamiento del control en cascada propuesto tiene mejor desempeño que el control en cascada convencional y que un diseño de control con un solo lazo de composición.

La combinación de las propiedades de los sensores a diferentes posiciones a lo largo del reactor provee al controlador con lazo de temperatura promedio rapidez de respuesta frente a perturbaciones en concentración y temperatura a la enţrada del reactor disminuyendo así los efectos en la concentración a la salida del reactor. 

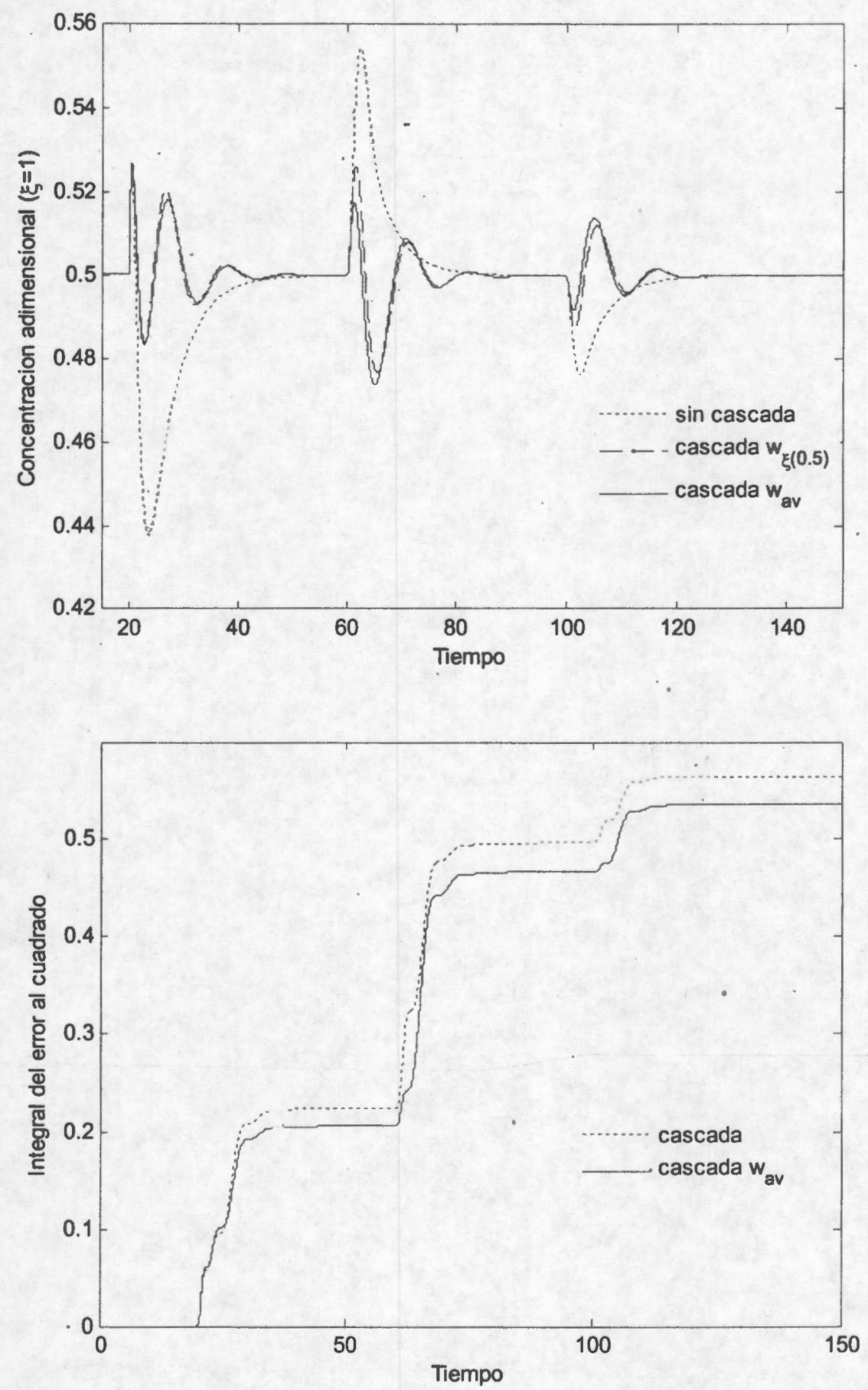

Figura 5-8 Desempeño de esquemas de control, un solo lazo de retroalimentación PI, control en cascada clásico y control en cascada propuesto: perfil cuasi-lineal, a) dinámica frente a perturbaciones $\mathrm{y}$ b) integral del error al cuadrado.

Para el caso con perfil del temperatura con hot spot los parámetros del controlador con un solo lazo de retroalimentación son $K_{c, \text {,y }}=4.2898$ y $\tau_{I, \text { ay }}=0.3762$. En la Figura 5-9a se 
muestra el desempeño de los controladores PI, control en cascada clásico y control en cascada con $w_{a v}$, observando que el control propuesto tiene un mejor desempeño (ver Figura 5-9b)
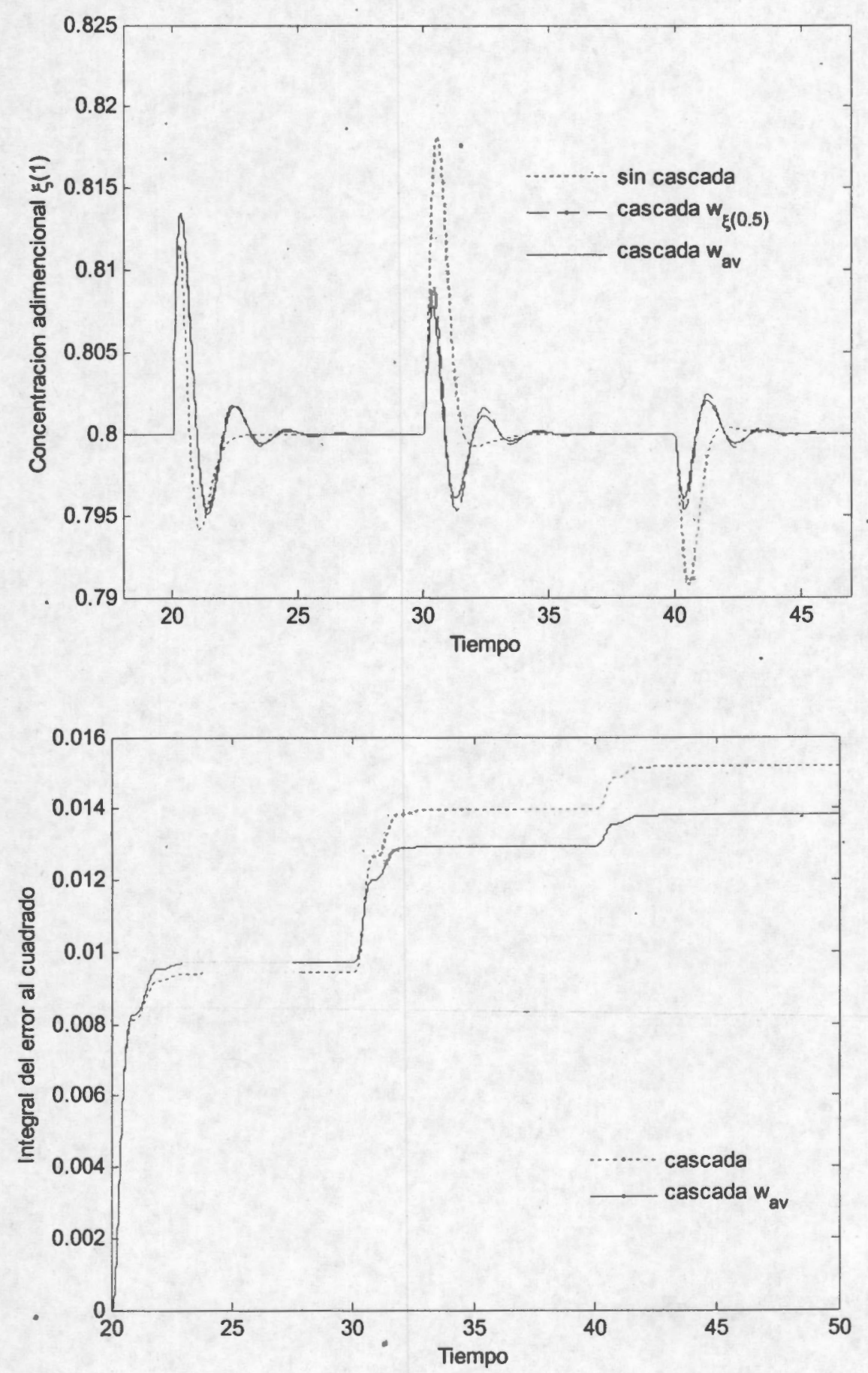

Figura 5-9 Desempeño de esquemas de control, un solo lazo de retroalimentación PI, control en cascada clásico y control en cascada propuesto: perfil con hot spot, a) dinámica frente a perturbaciones y b) integral del error al cuadrado.

Las simulaciones numéricas muestran exitosamente que el controlador con $\left(w_{a v}\right)$ tiene un mejor desempeño frente a perturbaciones de concentración y temperatura a la entrada del 
reactor que el control en cascada convencional y el controlador con un solo lazo de composición.
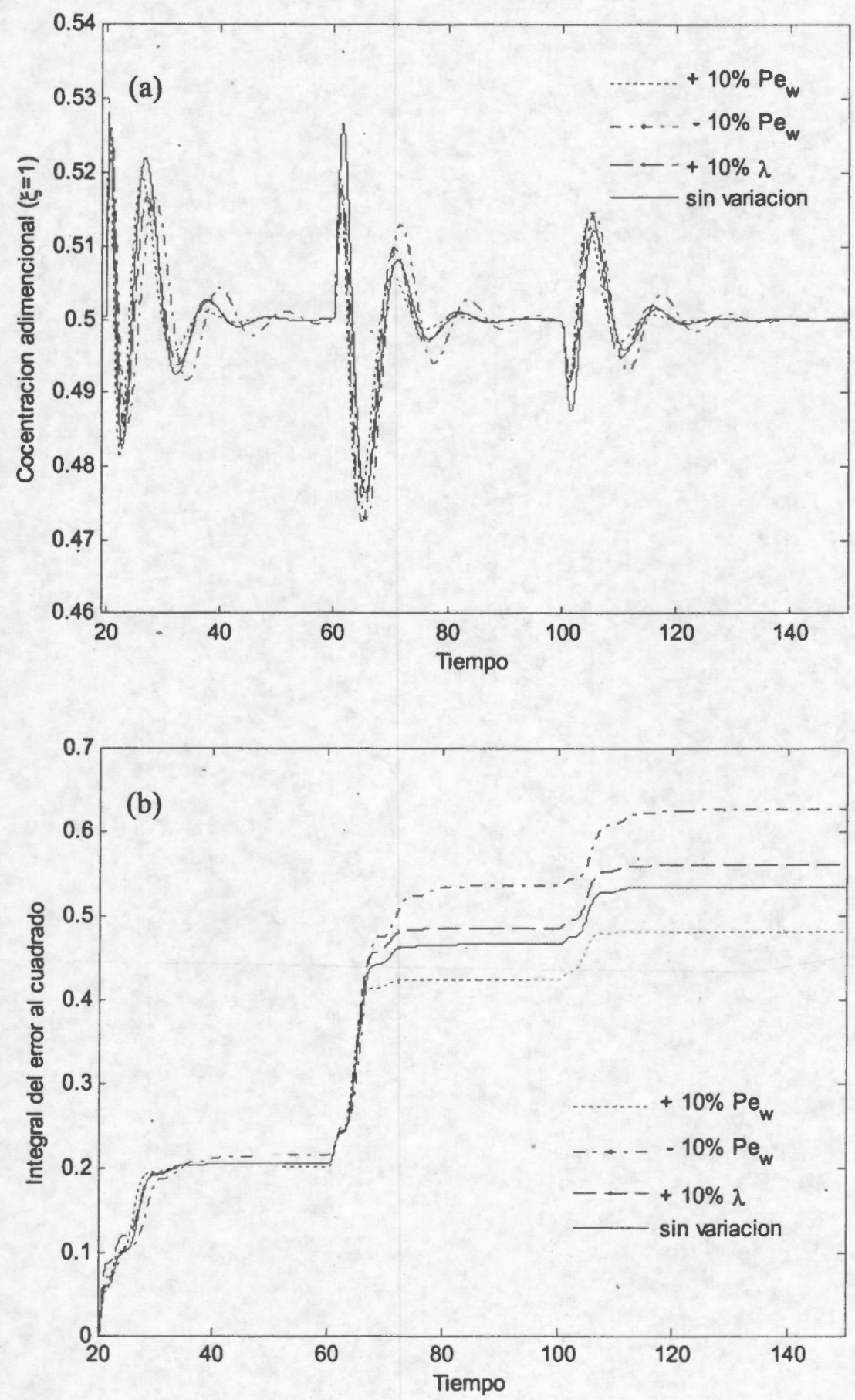

Figura 5-10 Pruebas de robustez al controlador propuesto, variación el los parámetros del modelo, perfil cuasi-lineal, a) regulación de concentración a $y_{r e f}=0.5$ frente a perturbaciones y b) integral del error al cuadrado. 
A pesar de las diversos comportamientos que puede presentar un reactor tubular, el desempeño de un controlador en cascada de composición-temperatura es mejor cuando el sensor de temperatura esta localizado en el centro del reactor tubular.

Un esquema de control en cascada composición-temperatura puede ser reforzado mediante el uso de múltiples sensores de temperatura (esto provee al controlador información del reactor a en diferentes posiciones) en el lazo secundario. Es de esperar que a mayor cantidad de sensores el desempeño del controlador deba ser mejor (mayor cantidad de información), sin embargo el problema de la asignación de peso a cada sensor se vuelve más complejo. Por otra parte, emplear solo dos sensores de temperatura nos lleva a discutir cual es la posición mas adecuada para colocar el sensor adicional, Urrea y col., (2007) proponen colocar el segundo sensor antes del hot spot, sin embargo nuestro estudio muestra que para dinámicas donde se presenta el hot spot es mas útil colocar el sensor de temperatura cerca a la salida que a la entrada del reactor y para dinámicas con perfil cuasi-lineal es mas conveniente colocar el sensor cerca de la entrada del reactor.

Acorde a lo anterior podemos sugerir que el número mínimo de sensores para fortalecer un controlador en cascada de composición-temperatura son tres, distribuidos simétricamente en la dirección axial del reactor. Mediante una adecuada distribución de peso en los parámetros de ponderación de los sensores garantizamos que el controlador tendrá un mejor desempeño que el control en cascada convencional.

\subsection{Pruebas de Robustez}

Uno de los problemas mas frecuentes de la simulación numérica frente la experimentación es la incertidumbre de los parámetros que modelan los procesos, frente a este hecho lo adecuado es contar con contralores robustos que permitan enfrentar cualquier cambio de los parámetros o las incertidumbres que se puedan presentar. A tal hecho se propone hacer variaciones en los parámetros que rigen al sistema para cuantificar la variación en la respuesta frente a perturbaciones del controlador con $w_{a v}$.

Las pruebas de robustez propuestas se hacen mediante la variación de los parámetros del sistema, para ambos casos de estudio, los cambios hechos al sistema son como siguen: $i$ ) variación del número de Peclet masico $\left(P e_{w}\right) \pm 10 \%$ del valor nominal y ii) aumento de $10 \%$ en la energía de activación adimensional $(\lambda)$.

Las simulaciones muestran que la respuesta del controlador frente a cambios en los parámetros del sistema no tiene grandes variaciones como se muestra en las Figuras 5-10a y 5-11a, perfil de temperatura cuasi-lineal y perfil con hot spot respectivamente. La integral del error no muestra cambios se ve en las Figuras $10 \mathrm{~b}$ y $11 \mathrm{~b}$, lo que nos sugiere que el controlador tienen buena robustez. 

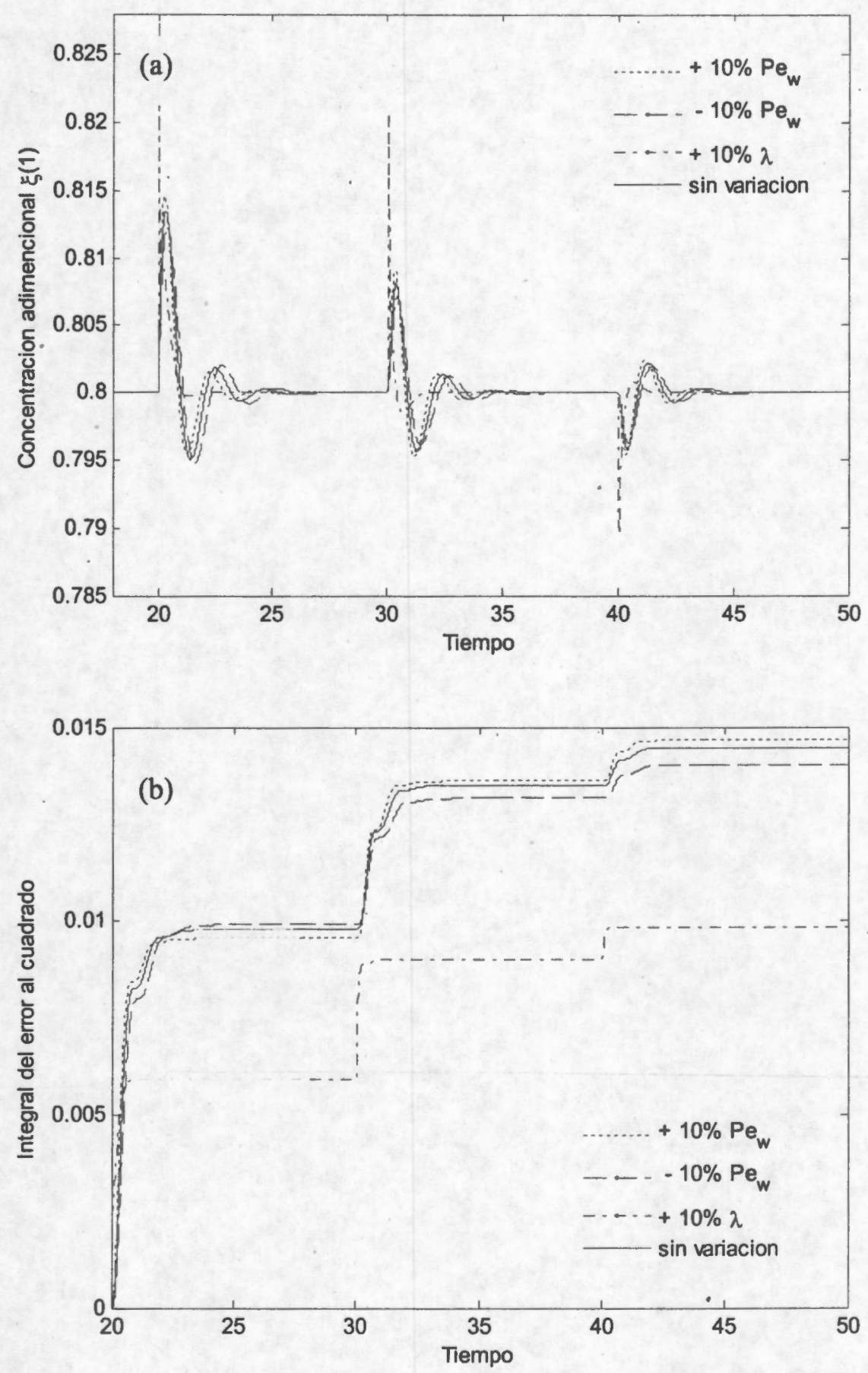

Figura 5-11 Pruebas de robustez al controlador propuesto, variación el los parámetros del modelo, perfil con hot spot, a) regulación de concentración a $y_{\text {ref }}=0.5$ frente a perturbaciones y b) integral del error al cuadrado 


\section{Conclusiones}

El trabajo se divide en dos partes importantes: $i$ ) el uso de Formulaciones de Ecuaciones Integrales (FEI) para la simulación numérica de modelos de reactores tubulares frente a los modelos clásicos de discretización numérica (DF y $\mathrm{CO}$ ) y ii) la implementación de un esquema de control en cascada que mejora las características de respuesta a perturbaciones en la alimentación de un esquema de control en cascada convencional.

En la primera parte del trabajo se realizo la transformación analítica del conjunto de ecuaciones diferenciales parciales que describen el comportamiento de un reactor tubular con dispersión axial a un conjunto de ecuaciones integro-diferenciales. La solución obtenida mediante (FEI) es comparada con diferencias finitas (DF) y colocación ortogonal (CO). El sistema es resuelto tanto para el estado estacionario como en estado dinámico, calculando el error relativo basado en la solución del sistema por DF empleando 2000 nodos, cuya solución se ha considerado como la solución "exacta". Para verificar la capacidad de aproximación numérica se ha resulto el sistema variando los números de Peclet en el rango de $10^{-1}-10^{2}$.

Los resultados muestran que el método de $\mathrm{CO}$ requiere de un número menor de nodos que DF y FEI, sin embargo existen regiones del número de Peclet donde $\mathrm{CO}$ tiene mayor error relativo que DF y FEI. Las FEI muestran menor error relativo a medida que el número de nodos empleados para discretizar el sistema es mayor, este comportamiento es constante para soluciones dinámicas o estáticas. El análisis estático muestra que FEI requiere de un menor mallado que DF para el mismo error relativo, sin embargo en algunos casos dinámicos los errores calculados para FEI son mayores que para DF empleando el mismo numero de nodos, pero en general cuando se emplean 500 nodos los valores del error relativo son semejantes o mejores que los de DF. El tiempo de computo requerido para la solución del sistema mediante FEI siempre es menor que el usado en DF.

Para la segunda parte se propone un esquema de control en cascada composicióntemperatura para el modelo del reactor tubular abordando dos casos de estudio: cuando la dinámica de la temperatura presenta un hot spot y cuando el perfil es cuasi-lineal. El sensor de temperatura para el lazo secundario del control en cascada se propone en $0.2,0.5$ y 0.8 adimensional a lo largo de la dirección axial del reactor, observando que el controlador con sensor de temperatura en 0.5 tiene el mejor desempeño, sin embargo se observo que el controlador con sensor de temperatura en 0.2 posee una respuesta mas rápida frente a perturbaciones en la concentración a la entrada del reactor y el controlador con sensor de temperatura en $\mathbf{0 . 8}$ posee mejor respuesta frente a perturbaciones de temperatura en la entrada del reactor. De esta forma se propone un controlador que explote las tres características de 
posición de los sensores de temperatura, el controlador propuesto emplea una temperatura promedio ponderada $\left(w_{a v}\right)$ virtual en el lazo secundario.

Mediante simulaciones numéricas verificamos exitosamente que el controlador con $\left(w_{a v}\right)$ tiene un mejor desempeño frente a perturbaciones de concentración y temperatura a la entrada del reactor que el control en cascada convencional.

Se realizaron pruebas de robustez al controlador en cascada propuesto para verificar el desempeño del controlador frente a incertidumbres en los valores de los parámetros que modelan al reactor tubular. Las simulaciones numéricas muestran que el controlador tiene buena robustez frente a cambios en los parámetros. 


\section{Bibliografía}

1. Agrawal N., Rangaiah G. P., Ray A. K. and Gupta S. K. (2006) "Multi-objective of the operation of an Industrial Low Density Polyethylene Reactor using Genetic Algorithm and Junping Gene Adaptations", Ind. \& Eng. Chem. Res. 45 (2006) 3182 3199.

2. Alvarez, J., Romagnoli, J.A., and Stephanopoulos, G. (1981). "Variable measurement structures for the control of a tubular reactor". Chem. Eng. Sci., 36 1695-1712.

3. Alvarez-Ramirez J., Suarez R. and Morales A., (2000), "Cascade control for a class of uncertain nonlinear systems: a backstepping approach", Chem. Eng. Sci. 3209-3221

4. Alvarez Ramirez J., Puebla H. and Ochoa-Tapia J., (2001), "Linear boundary control for a class of nonlinear PDE process", Systems \& Control Letters 44, 395-403

5. Álvarez-Ramírez J., Valdez-Parada F., Álvarez J. and Ochoa-Tapia A. (2007) "A Green Formulation for finite-differences Schemes”, Chem. Eng. Sci., 62 3083-3091.

6. Aris, R., (1969). "Elementary Chemical Reactor Analysis". Prentice Hall, Inc., Engiewood Cliffs, N.J.

7. Brambilla A. and Semino D., Scali C. (1994), "Design and Control Selection of Cascade Loop in Distillation". IFAC Workshop on Integration of Process Design and Control, Baltimore, MD, p. 171.

8. Brambilla A. and Semino D. (1992) "Nonlinear Filter in Cascade Control Schemes", Ind. Eng. Chem. Res., vol. 31, pp. 2694-2699.

9. Christofides P. D., (2002) "Special volume on "control of distributed parameter systems". Comp. \& Chem. Eng., 26, 939-940.

10. Christofides P.D. (2001). "Nonlinear and robust control of partial differential equation system: methods and applications to transport-reaction processes". Systems and Control: Foundations and Applications Series. Birkhauser, Boston.

11. Christofides, P. D., Teel, A. R. and Daoutidis. P. (1995). "Robust semi-global output tracking for nonlinear singularly perturbed systems". Proceedings of the $34^{\text {th }}$ Conference on Decision \& Control. New Orleans, LA - December 1995. 
12. Costa E., Lage P. and Biscaia P. Jr. (2003) "On Numerical solution and optimization of styrene polymerization in tubular reactors", Comp. \& Chem. Eng., 27 1591-1604.

13. Danckwerts P V. (1953), "Continuous flow systems. Distribution of residence times". Chem. Eng. Sci. 2, 1-13

14. Dixit R.S. and Tavlarides L.L. (1982). "Integral Method of analysis of FischerTropsch synthesis reactions in a catalyst pellets". Chem. Eng. Sci. 37 539-544

15. Dochain D., Babary J. P. and Tali-Maamar N. (1992). "Modeling and adaptative control of nonlinear distributed parameters bioreactors via orthogonal collocation". Automatic, 28 873-883

16. Dubljevic S., El-Farra, N. H., Mhaskar, P. and Christofides, P. D. (2004). "Predictive control of parabolic PDEs with State and Control Constraints". Proceeding of the 2004 American Contro Confeefnce, Boston, Massachesetts, June - July, 2004.

17. Edgar T. F., Heeb R. C. and Hougen J. O. (1982), "Computer-aided Process control system design using interactive graphics", Com. Chem. Eng. 5(4), 225.

18. Finlayson, B. A. (1980), "Nonlinear Analysis in Chemical Engineering". McGraw Hill. New York, USA.

19. Foss S., Edmunds, J. and Kouvarltakis B., (1980) "Multivariable control system for two-bed reactors by the characteristic locus method." Ind . Eng. Chem. Fundam. 19. 109-117.

20. Froment, G.F. and Bischoff, K.B. (1990) "Chemical Reactor Analysis and Design". Second Edition, John Wiley and Sons, Inc.

21. Georgakis, C., Aris, R., and Amundson, N.R. (1977). "Studies in the control of tubular reactors-I: General considerations". Chem. Eng. Sci., 32 1359-1369.

22. Georgakis, C., Aris, R., and Amundson, N.R. (1977). "Studies in the control of tubular reactors-II: Stabilization by modal control”. Chem. Eng. Sci., 32 1371-1379.

23. Georgakis, C., Aris, R., and Amundson, N.R. (1977). "Studies in the control of tubular reactors-III: Stabilization by observer design". Chem. Eng. Sci., 32 1381-1387.

24. Greenberg M. D. (1971), “Applications of Green's Functions in Science and Engineering”. Prentice Hall, New York

25. Haberman R. (2003) "Partials Derivatives Equations with Fourier series and boundary problems". Prentice Hall, USA

26. Hanczyc, N., and Palazoglu, A., (1995), "Sliding mode control of nonlinear distributed parameter chemical processes". Ind. Eng. Chem. Res., 34, 557-566.

27. Hougen J. O., (1979). "Measurement and control applications: Instrument society of America", Pittsburg PA. 
28. Hua X. and Jutan A., (2000), "Nonlinear Inferential Cascade Control of Exothermic Fixed-Bed Reactors, AICHE, 46, No. 5

29. Irvin J. and Mullineux N., (1959). Mathematics in physics and engineering, Academic Press, New York

30. Jiménez-Islas, H. (1999). "Modelamiento Matemático de los Procesos de Transferencia de Momentum, Calor y Masa". Tesis Doctoral, Universidad Autónoma Metropolitana Unidad Iztapalapa, México D. F.

31. Jiménez-Islas, H., López-Isunza, F. and Ochoa-Tapia, A. J. (1994). Natural Convection in a Porous Media Enclosed in a Vertical Cylinder: The Numerical Solution by Double Orthogonal Collocation". Proceedings of 7th International Symposium on Transport Phenomena in Manufacturing Processes, pp 41-46.

32. Jury F. D., (1973). Fundamentals of three-mode controllers; Fisher Controls Company: Technical monograph, No 28.

33. Khalid A., Redhoune H. and Mostar S., (2003) "Numerical analysis of reactiondiffusion-convection systems", Computers \& Chem. Eng. 27 579-594

34. Krishnaswamy P. R. and Rangaiah G. P. (1990). "When to used cascade control", Ind. Eng Chem. Res. 29, 2163-2166.

35. Lee Y. and Park S. (1998). PID "Controller tuning to Obtain Desired Closed Loop response for cascade control systems". Ind. Eng. Chem. Res. 37 1859-1865.

36. Lefervre, L., Dochain, D. and Feyo de Azevedo, S., \& Magnus A. (2000) "Optimal selection of orthogonal polynomials applied to the integration of chemical reactor equations by collocation methods", Computers \& Chem. Eng. 24 2571-2588

37. Li M. and Christofides P.D., (2007) "An input/output approach to the optimal transition control of a class of distributed Chemical reactors", Chem. Eng. Sci. 62 , 2979-2988.

38. Luyben, W. L., (1990), "Process Modeling, Simulation and Control for Chemical Engineers", 2nd Ed. McGraw-Hill, Singapore

39. Morari, M. and Zafiriou, E. (1989). "Robust process control", Prentice-Hall, New York.

40. Mukkavilli S. and Tavlarides L. T (1987). "Integral Method of analysis for chemical reaction in no isothermal finite cylindrical catalyst pellet-I Dirichlet Problem". Chem. Eng. Sci., 42 27-33.

41. Mukkavilli S. and Tavlarides L, T (1987). "Integral Method of analysis for chemical reaction in no isothermal finite cylindrical catalyst pellet-II Robin Problem". Chem. Eng. Sci., 42 35-40. 
42. Onyejekwe, O (1996). "A Green element description of transfer mass in reacting systems Part-B". Numerical heat transfer, 30 483-498.

43. Onyejekwe, O (2002). "Green element solutions of nonlinear diffusion-reaction model". Comp. \& Chem. Eng., 26 423-427.

44. Patwardhan A. A., Wright G. T. and Edgar T. F. (1992) "Nonlinear model predictive control of distributed-parameters systems". Chem. Eng. Sci. 47 721-735.

45. Rice R. and Do D., (1995) "Applied mathematics and modeling for chemicals Engineers", John Wiley \& Sons, Inc., New York USA.

46. Roach, G. F. (1982). "Green's Functions". Second Edition, Cambridge University Press, Great Britain.

47. Russo P. and Bequette W. B. (1997), "State-Space versus Input/Output Representation for Cascade Control of Unstable System", Ind. Eng. Chem Res. vol. 36, pp. 22712278.

48. Semino D. and Brambilla A. (1996), "An Efficient Structure for Parallel Cascade Control". Ind. Eng. Chem. Res. vol. 35, pp. 1845-1852.

49. Shen S. Y. and Yu C. C. (1990), "Selection of Secondary Measurement for Parallel Cascade Cantrol". AIChE J., 36, 1267

50. Skogestad Sigurd (2003). "Simple analytic rules for model reduction and PID controllers tuning". Journal of Process control. 13 291-309.

51. Smith, G. D. (1978) "Numerical Solution of Partial Differential Equations: Finite Difference Methods". Clarendon Press. Oxford, G.B

52. Smith, J. M. (1986) "Chemical Engineering Kinetics". 3er Edition, Mc Graw Hill USA

53. Smith T. and Corripio (1997), "Principles and practice of automatic process control", second edition, John Wiley \& Sons Inc. New York.

54. Stanbeland, B.E.. and Foss, A S (1970). "Control of a fixed-bed chemical reactor". Ind. Eng. Chem. Fundam., 9 38-43.

55. Stephanopoulos G. (1984), "Control Systems with Multiple Loops in Chemical Process Control". Ed. Prentice Hall, Englewood Cliffs N. J. USA, Capítulo 20.

56. Urrea R., Castellanos-Sahagun E., Alvarez J., and Alvarez-Ramirez J., (2006). Distillate "Cascade composition control using a two-temperature measurement secondary component", Ind. Eng. Chem. Res. 6828-6841

57. Urrea R., Alvarez J. and Alvarez-Ramirez J.,(2008), "Linear PI temperaturecomposition cascade control for tubular reactor", Chemical Engineering Communications, 1957 803-820 
58. Valdez-Parada F., Sales-Cruz M., Ochoa-Tapia A. and Álvarez-Ramírez J. (2007). "On Green's Function Methods to Solve Nonlinear Reaction-Diffusion Systems", Comp. \& Chem. Eng. In press

59. Valdez-Parada F., Sales-Cruz M., Ochoa-Tapia A. and Álvarez-Ramírez J. (2007). "A Green's Function Approach for Solving Nonlinear Reaction-Diffusion-Convection Systems". En revision

60. Villadsen, J. V. and Stewart, W. E. (1967) "Solution of Boundary-Value Problems by OrthogonarCollocation”. Chem. Engng. Sci., Vol. 22, 1483-1501.

61. Wallman, P.H.,Silva, J.M., and Foss, AS., (1979) "Multivariable integrals controls for fixed bed reactors". Ind. Eng. Chem. Fundam., 18, 392-399.

62. Wu W. and Chen C. (2007) "Measurement-Based Optimization and Predictive Control for an Exothermic Tubular Reactor System", Ind. Eng. Chem., 46 2064-2076.

63. Yu, C. C. (1988), "Design of Parallel Cascade Control for Disturbance Rejection". AIChE J., 34, 1833. 


\section{Apéndice}

En este apartado del trabajo se presenta material útil para un mejor entendimiento acerca de temás que aborda la presente tesis tales como: propiedades de la función impulso, solución numérica de reactores tubulares mediante diferencias finitas y colocación ortogonal.

\section{A.1 Propiedades de la función Impulso}

La función de Green refleja la influencia de cada posición x 0 de la fuente sobre la solución, es comportamiento es asociado con la función impulso o también llamada delta de Dirac. Por ello presentamos las propiedades mas importantes de la función impulso que se relacionan con la función de Green.

Delta de Dirac: Nuestra fuente $f(x)$ representa una fuerza aplicada a nuestro sistema en todos los puntos en una combinación lineal de pulsos unidad de duración $\Delta x$ (ver Figura A-1)

$$
f(x) \approx \sum_{i} f\left(x_{i}\right) \phi
$$

donde $\phi$ es el pulso unidad que comienza en $x=x_{i}$, la Ecuación A-1 semeja la definición de la integral, asi que dividiendo y multiplicando por $\Delta x$ tenemos:

$$
f(x)=\frac{\lim }{\Delta x \rightarrow 0} \sum_{i} \frac{f\left(x_{1}\right) \phi}{\Delta x} \Delta x
$$

En la Figura $A-2$ se representa un pulso rectangular de anchura $\Delta x$ y altura $1 / \Delta x$ que, por tanto, tiene área unidad. En el límite cuando $\Delta x \rightarrow 0$, este pulso se aproxima a otro infinitamente concentrado que debería ser cero en todas partes excepto en $\infty$ en $x=x_{\text {, }}$, pero con área unidad:

$$
\delta\left(x-x_{i}\right)= \begin{cases}0 & x \neq x_{i} \\ \infty & x=x_{i}\end{cases}
$$




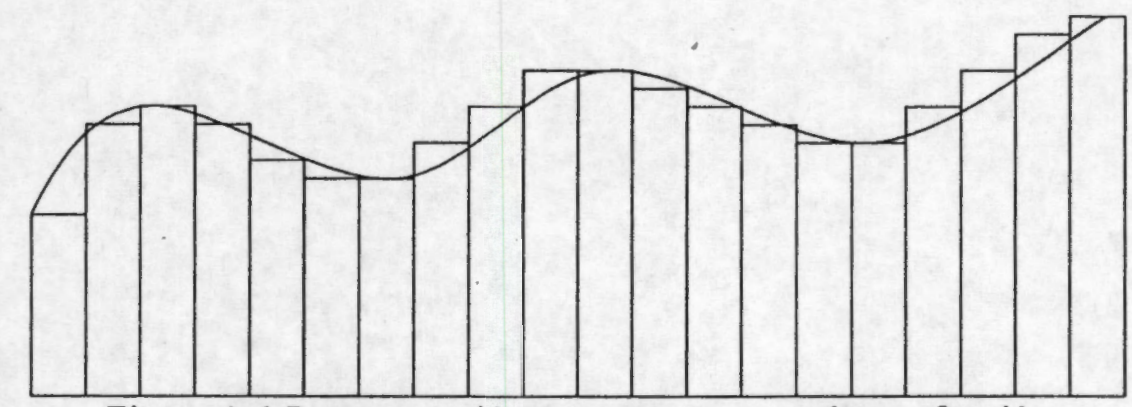

Figura A-1 Representación constante a trozos de una función

Podemos pensar en $\delta\left(x-x_{i}\right)$ como una fuente concentrada en el único punto $x=x_{i}$. De acuerdo con la Ecuación $\mathrm{A}: 2$, tenemos

$$
f(x)=\int f\left(x_{i}\right) \delta\left(x-x_{i}\right) d x_{i}
$$

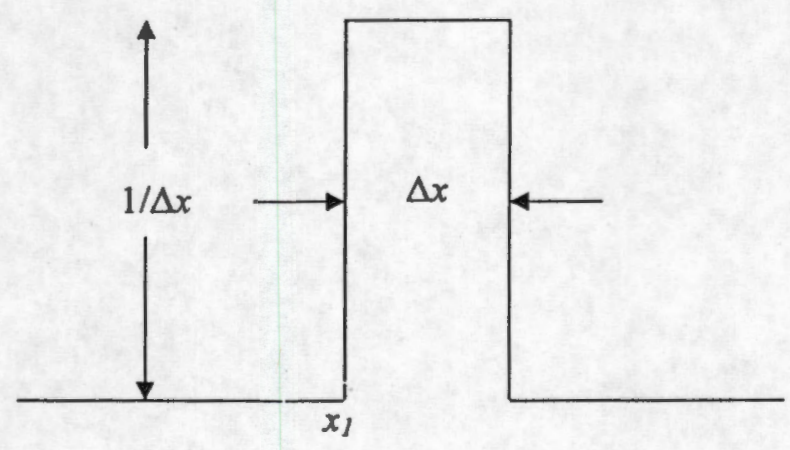

Figura A-2 Pulso rectangular con área unidad

Puesto que $\delta\left(x-x_{i}\right)$ no es una función, la definimos como un operador con la propiedad de que para cualquier función continua $f(x)$ :

$$
f(x)=\int_{-\infty}^{\infty} f\left(x_{i}\right) \delta\left(x-x_{i}\right) d x_{i}
$$

$\delta\left(x-x_{i}\right)$ es la llamada función delta de Dirac. Está tan concentrada que, al integrarla con cualquier función continua $f\left(x_{i}\right)$, la filtra para devolver el valor de esta en $x=x_{i}$. Otras propiedades importantes de la función delta de Dirac son: que tiene área de unidad,

$$
1=\int_{-\infty}^{\infty} f\left(x_{i}\right) \delta\left(x-x_{i}\right) d x_{i}
$$


que es par,

$$
\delta\left(x-x_{i}\right)=\delta\left(x_{i}-x\right)
$$

La delta de Dirac es también la derivada de la función de salto de Heaviside, $H\left(x-x_{i}\right)$

$$
H\left(x-x_{i}\right)= \begin{cases}0 & x<x_{i} \\ 1 & x>x_{i}\end{cases}
$$

\section{A.2 Diferencias Finitas}

El método de diferencias finitas está dotado con la simplicidad de su principio (la representación de las derivadas de una función, en términos de series de Taylor finitas), y la facilidad de formulación e implementación sobre computadoras digitales. Las desventajas incluyen: la dificultad en implementar las condiciones frontera sobre fronteras irregulares, y la dificultad en representar con precisión dominios irregulares.

El método de diferencias finitas trata con puntos específicos dentro un dominio denominados puntos malla. El dominio está dividido en intervalos equidistantes, aunque tal consideración de intervalos iguales no es necesaria.

Si se supone que se tiene una función continua $\theta(z)$, al usar una expansión de Taylor se pueden deducir fórmulas en diferencias para la primer y segunda derivada representados por:

$$
\begin{aligned}
& \theta_{i+1}=\theta_{i}+\theta_{i}^{\prime} \Delta x+\theta_{i}^{\prime \prime} \frac{\Delta x^{2}}{2 !}+\theta_{i}^{m} \frac{\Delta x^{3}}{3 !}+\ldots . \\
& \theta_{i-1}=\theta_{i}-\theta_{i}^{\prime} \Delta x-\theta_{i}^{\prime \prime} \frac{\Delta x^{2}}{2 !}-\theta_{i}^{m} \frac{\Delta x^{3}}{3 !}-\ldots .
\end{aligned}
$$

al reordenar $y$ dividir por $\Delta \mathrm{x}$ se obtienen dos expresiones para la primer derivada

$$
\begin{aligned}
& \theta_{i}^{\prime}=\frac{\theta_{i+1}-\theta_{i}}{\Delta x}+\theta_{i}^{\prime \prime} \frac{\Delta x}{2 !}+\theta_{i}^{m} \frac{\Delta x^{2}}{3 !}=\frac{\theta_{i+1}-\theta_{i}}{\Delta x}+\vartheta(x) \\
& \theta_{i}^{\prime}=\frac{\theta_{i}-\theta_{i-1}}{\Delta x}-\theta_{i}^{\prime \prime} \frac{\Delta x}{2 !}-\theta_{i}^{\prime \prime} \frac{\Delta x^{2}}{3 !}=\frac{\theta_{i}-\theta_{i-1}}{\Delta x}-\vartheta(x)
\end{aligned}
$$

La expresión (A-10) se conoce como la primera deriva en diferencias hacia adelante y (A11) hacia atrás. $\vartheta(x)$ es el error de truncamiento. La segunda derivada se obtiene de la serie de Taylor (A-9), y tiene la forma:

$$
\theta_{i}^{*}=\frac{\theta_{i+1}-2 \theta_{i}+\theta_{i-1}}{\Delta x^{2}}+\vartheta(x)
$$


El método de diferencias finitas tiene la ventaja de su fácil formulación, sin embargo es necesario en algunos casos un elevado número de puntos malla para obtener una alta precisión.

Cuando la solución en los puntos malla se conoce, la solución en otra posición se encuentra por interpolación lineal. Para el caso de los reactores tubulares, las ecuaciones diferenciales parciales se discretizan en la posición espacial, lo cual conduce a un sistema de ecuaciones diferenciales ordinarias (una para cada punto malla en cada variable dependiente). Estas ecuaciones pueden ser integradas por distintos métodos, por ejemplo, esquemas de integración explicita, tales como los métodos de Runge-Kutta o el método de Euler.

\section{A.3 Colocación Ortogonal}

La colocación ortogonal es un caso especial del método de residuos ponderados, donde los puntos donde el residual es cero, corresponden a las raíces de polinomios ortogonales como los de Jacobi, Hermite, Laguerre, etc., que se emplean como funciones de prueba. Con este método es posible convertir EDO a sistemas de ecuaciones algebraicas, que se pueden resolver por Newton- Raphson, Broyden, técnicas de seguimiento homotópico, relajación no lineal, etc. Las ecuaciones diferenciales parciales (EDP) del tipo parabólico, se convierten a sistemas de EDO con valores iniciales, que se pueden resolver por metodos de integración como los de Runge-Kutta o similares. Los polinomios de Jacobi se generan a partir de la siguiente expresión:

$$
\int_{0}^{1} x^{\beta}(1-x)^{\alpha} P_{j}(x) P_{N}(x) d x=0
$$

Si $\alpha=0$ y $\beta=0$, se obtienen los polinomios transformados de Legendre. Sí se propone que la solución de aproximación tiene la forma:

$$
y=\sum_{i=1}^{N+2} d_{i} x_{j}^{i-1}
$$

Obteniendo la primera y segunda derivada de (A-14):

$$
\begin{aligned}
& \frac{d y}{d x}=\sum_{i=1}^{N+2} d_{i}(i-1) x_{j}^{1-2} \\
& \frac{d^{2} y}{d x^{2}}=\sum_{i=1}^{N+2} d_{i}(i-1)(1-2) x_{j}^{i-3}
\end{aligned}
$$

en donde, el término $x_{j}$ representa las raíces del polinomio ortogonal seleccionado en el dominio prefijado. Ahora si: 


$$
\begin{aligned}
& y=Q d \\
& \frac{d y}{d x}=C d \\
& \frac{d^{2} y}{d x^{2}}=D d
\end{aligned}
$$

en donde

$$
\begin{aligned}
& Q_{j i}=x_{j}^{(i-1)} \\
& C_{j i}=(i-1) x_{j}^{(i-2)} \\
& D_{j i}=(i-1)(i-2) x_{j}^{(i-3)}
\end{aligned}
$$

resolviendo para d, se tiene que:

$$
\begin{aligned}
& d=Q^{-1} y \\
& \frac{d y}{d x}=C Q^{-1} y=A y \\
& \frac{d^{2} y}{d x^{2}}=D Q^{-1} y=B y
\end{aligned}
$$

Siendo A y B las matrices de colocación que aproximarán a la primera y segunda derivada respectivamente.

Hay que tener en mente que pueden existir mas de un vector solución cuando el sistema algebraico es no lineal, por lo que la solución que se obtenga se le deberá ser evaluada como factible termodinámicamente en función del problema que se está analizando (Jiménez-Islas, 1988).

La colocación ortogonal discretiza con mayor precisión las ecuaciones diferenciales ordinarias y parciales, ya que su dominio es de frontera a frontera, mientras que en los métodos de diferencias finitas, su dominio está limitado a los nodos adyacentes.

En circunstancias similares, la precisión obtenida por el mallado de colocación ortogonal equivale, en términos generales, a dos o tres veces el mallado por diferencias finitas (Finlayson, 1980, Jiménez-Islas et al, 1994), por lo que la colocación ortogonal es un método eficiente para la solución de ecuaciones diferenciales ordinarias y parciales, tanto para valores iniciales como con valores a la frontera. 